\title{
Distortion-Rate Bounds for Fixed- and Variable-Rate Multiresolution Source Codes
}

\author{
Michelle Effros, Member, IEEE
}

\begin{abstract}
The source coding theorem for stationary sources describes the optimal performance theoretically achievable by fixed- and variable-rate block quantizers. The source coding theorem may be generalized by considering the problem of multiresolution or successive refinement source coding, which is the topic of this work. Given a distortion vector $\left(D_{1}, \cdots, D_{L}\right)$, this work describes the family of achievable rate vectors $\left(R_{1}, \cdots, R_{L}\right)$ for describing a stationary source at $L$ resolutions, where the description at the first resolution is given at rate $R_{1}$ and achieves an expected distortion no greater than $D_{1}$, the description at the second resolution includes both the first description and a refining description of rate $R_{2}$ and achieves expected distortion no greater than $D_{2}$, and so on. The work includes performance bounds for both fixed- and variable-rate source codes on discrete-time stationary ergodic sources and discrete-time stationary nonergodic sources for any integer number of resolutions $L \geq 1$. For $L=1$, the source coding theorems for stationary sources result. For $L>1$, the results extend previous theorems for discrete-alphabet memoryless sources.
\end{abstract}

Index Terms-Embedded, ergodic and nonergodic source coding theorems, multiuser information theory, progressive transmission, successive refinement.

\section{INTRODUCTION}

W ITH advances in communications media and technologies come corresponding needs for communication techniques that take full advantage of the capabilities particular to those technologies. A prime example of such an area of growth is the medium of internet communications. With the growth of Internet communications comes an increased need for techniques whereby a single user can simultaneously communicate the same information to a wide array of other users with vastly varying bandwidth resources, computational capabilities, and performance requirements. Along with a variety of other factors, this need has helped inspire a surge in interest in multiresolution or progressive transmission source coding.

Multiresolution source codes are data compression algorithms in which simple, low-rate source descriptions are embedded in more complex, high-rate descriptions, as illustrated in Fig. 1. Use of multiresolution source codes allows users with severe bandwidth constraints or low performance re-

Manuscript received April 29, 1998; revised March 1, 1999. This work was supported in part by NSF under Grant MIP-9501977 and under a Grant from the Charles Lee Powell Foundation. The material in this paper was presented in part at the IEEE International Symposium on Information Theory, Cambridge, MA, August 16-21, 1998.

The author is with the Department of Electrical Engineering, MC 136-93, California Institute of Technology, Pasadena, CA 91125 USA.

Communicated by N. Merhav, Associate Editor for Source Coding.

Publisher Item Identifier S 0018-9448(99)05881-2.

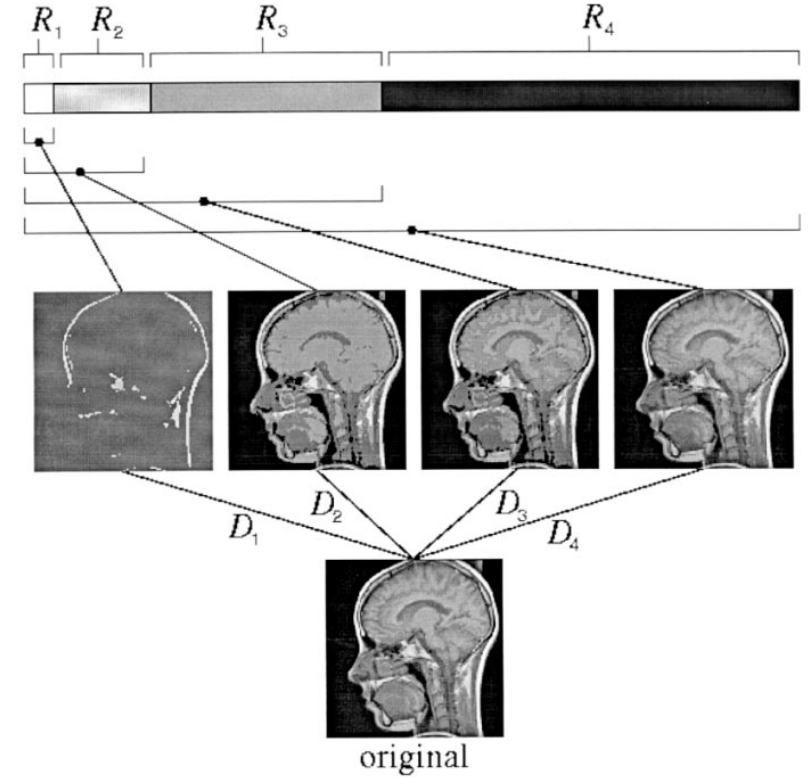

Fig. 1. A four-resolution description of a magnetic resonance brain scan. Decoding the first $R_{1}$ bits per symbol of the binary description yields a reproduction with distortion $D_{1}$. Decoding an additional $R_{2}$ bits per symbol, for a total rate of $R_{1}+R_{2}$ bits per symbol, yields a reproduction of distortion $D_{2}$, and so on.

quirements to achieve a low-quality data representation by only incorporating a fraction of the original coded bit stream. Users with greater capabilities or needs can achieve more precise data representations by using larger fractions of the same bit stream. Further, users uncertain of their precision needs can progressively reconstruct the data to higher and higher accuracy-stopping the communication process when the desired accuracy is achieved. Such coding techniques are extremely valuable in any application where multiple source descriptions at varying levels of precision are required.

While interest in multiresolution or progressive transmission source coding has spawned an enormous amount of research into practical coding algorithms (e.g., [1]-[4]), the theory behind these codes has, until recently, attracted much less attention. As a result, this discussion begins with the literature associated with single-resolution source codes.

In single-resolution source coding, a source is broken into contiguous vectors of length $N$ symbols, and each source vector is mapped to a binary description with some average per-symbol description length, say $R$. The source coding theorem and its converse describe the optimal distortion $D(R)$ 


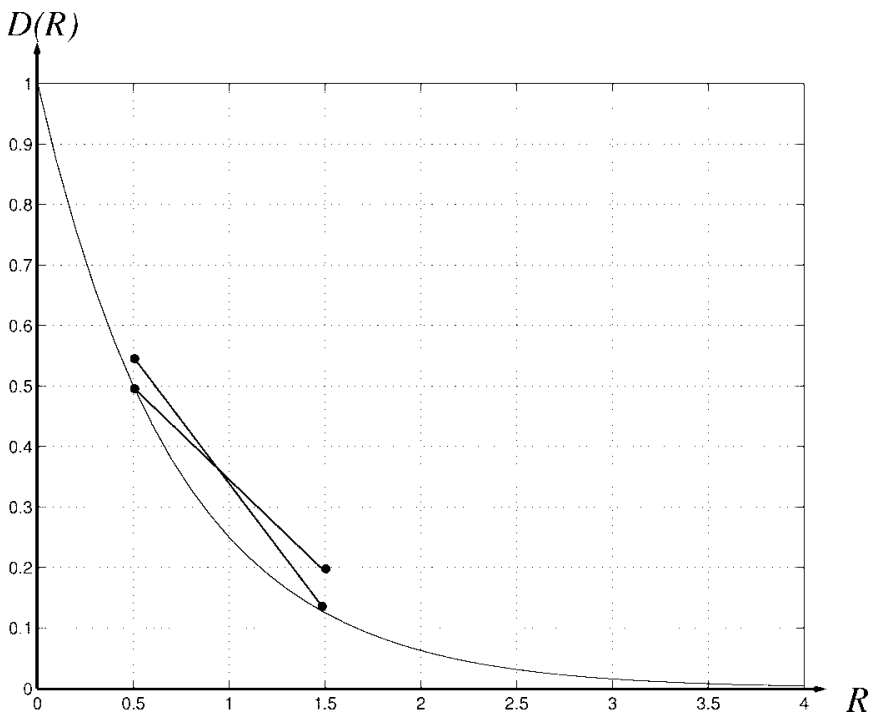

Fig. 2. The distortion-rate bound $D(R)$ describes the lowest expected distortion achievable at expected rate $R$ on a known source. Thus $D(0.5)$ is the lowest distortion achievable at rate 0.5 bits per symbol and $D(1.5)$ is the lowest distortion achievable at rate 1.5 bits per symbol in the above graph. The distortion-rate bound does not, however, describe the optimal achievable performance for an $L$-resolution code with $L>1$. For example, the distortion-rate function does not describe the lowest distortion achievable by adding 1 bit per symbol to a code achieving point $(0.5, D(0.5))$ in the above graph. Similarly, the distortion-rate function does not describe the lowest distortion achievable by reading only the first 0.5 bits per symbol from a code achieving point $(1.5, D(1.5))$ in the above graph. Further, the distortion-rate function does not describe all possible values of distortions $D_{1}$ and $D_{2}$ such that points $\left(0.5, D_{1}\right)$ and $\left(1.5, D_{2}\right)$ are achievable by the firstand second-resolution descriptions of a single two-resolution code.

theoretically achievable at the given average rate $R$. Source coding theorems and their converses for stationary ergodic and stationary nonergodic sources on complete separable metric spaces, known as Polish alphabets, appear in [5, Theorems 7.2.4, 7.2.5] and [6], respectively.

According to the source coding theorem, given an average rate of $R$ bits per symbol, the distortion $D(R)$ is achievable. Imagine now stripping off a fraction of the above $R$ bits per symbol to leave some lower average rate of $r$ bits per symbol. While distortion-rate theory bounds by $D(r)$ the optimal distortion theoretically achievable with these $r$ bits per symbol, the source coding theorem does not prove the achievability of this bound subject to our constraint on the higher resolution code. Further, given a rate $r$ source code achieving, to arbitrary accuracy, the distortion-rate bound $D(r)$, the source coding theorem bounds the best possible distortion achievable by appending $R-r$ bits per symbol to the given rate- $r$ description, but does not prove this bound to be achievable. An illustration of this observation appears in Fig. 2.

In [7], Gray and Wyner bound the achievable rate region of a simple, three-node network. The network consists of one transmitter sending information over three different (noiseless) channels to two receivers. The output of channel 0 is seen by both receivers while the output of channel 1 is seen only by the first receiver and the output of channel 2 is seen only by the second receiver. The transmitter's goal is to describe random variable $X$ to the first receiver and random variable $Y$ to the second receiver using the three channels. Gray and Wyner bound the collection of achievable rate triples $\left(R_{0}, R_{1}, R_{2}\right)$ for describing $X$ and $Y$ to arbitrary fidelity constraints $D_{X}$ and $D_{Y}$. When $X=Y$ and one of the private channels is not used (e.g., $R_{2}=0$ ), the network communication problem simplifies to the multiresolution source coding problem. That is, given $X=Y, R_{2}=0$, and $D_{X}<D_{Y}$, finding the achievable rate triples $\left(R_{0}, R_{1}, 0\right)$ is equivalent to finding the achievable rate pairs for describing a source to two resolutions, where the first-resolution description describes $X$ at rate $R_{0}$ with an expected distortion $D_{Y}$ and the second-resolution description supplements the shared description of $X$ with an additional $R_{1}$ bits per symbol to achieve an expected distortion $D_{X}$. In this scenario, the Pangloss bound described by Gray and Wyner is met by any code such that $D_{X}=D\left(R_{0}+R_{1}\right)$. Thus according to the above definition, any code for which the second-resolution coding performance sits on the distortionrate bound meets the Pangloss condition.

In [8] and [9], Koshelev introduces the notion of successive refinement codes, a concept closely related to Gray and Wyner's Pangloss bound codes. Here, a source is said to be successively refinable if a code giving a nested, tworesolution description of that source can always achieve the distortion-rate bound at both rates. Other related problems include the problem of source coding with side information [10] — which gives the minimum rate necessary to describe a source at distortion $D$ when side information $Y$ is present (but typically forces $Y$ to be independent and identically distributed (i.i.d.) samples from the same distribution) and the multiple descriptions problem [11], [12], in which a sender describes the same sequence of random variables over a variety of channels, and the receiver decodes using the outputs of those channels that do not fail in a given transmission.

In [13] and [14], Equitz and Cover prove that successive refinement is achievable for i.i.d. sources if and only if $X$, $\hat{X}_{2}$, and $\hat{X}_{1}$ form a Markov chain, where $\hat{X}_{1}$ and $\hat{X}_{2}$ represent the low- and high-resolution reproductions of $X$, respectively. They then go on to give three examples of sources that are successively refinable and a fourth example to show that there exist discrete-alphabet i.i.d. sources that are not successively refinable. An example of a simple continuous random variable that is not successively refinable appears in [15]. In [16], Rimoldi generalizes Equitz and Cover's result by finding the achievable rate pairs for a given pair of distortions in a two-resolution code. He then generalizes those results to $L$ resolution codes for $L>2$. Like [13] and [14], [16] treats discrete-alphabet memoryless sources.

This work contains a derivation of the achievable distortionrate region for $L$-resolution source codes for stationary ergodic and stationary nonergodic sources on Polish alphabets. The work can be viewed as an extension from one to $L$ resolutions of the source coding results in [5] and [6] or an extension of [16] from discrete-alphabet memoryless sources to arbitrary stationary sources on Polish alphabets. The variable-rate and variable-distortion Lagrangian approach made popular by [17] and employed throughout [6] again plays a crucial role in this work.

The resulting bounds describe the optimal performance theoretically achievable by both fixed- and variable-rate mul- 
tiresolution source codes and lend insight into the optimal design of new, practical codes, described at length in [18] and [19].

\section{PRELIMINARIES}

Let $\left(A^{\infty}, \mathcal{A}^{\infty}, \mu, T\right)$ be a stationary dynamical system with Polish alphabet $A$. That is, let $A$ be a complete, separable metric space, let $\mathcal{A}$ be the Borel $\sigma$-algebra generated by the open sets of $A$, let $A^{\infty}$ be the set of one-sided sequences $x=$ $\left(x_{1}, x_{2}, \cdots\right)$ from $A$, let $\mathcal{A}^{\infty}$ be the $\sigma$-algebra of subsets of $A^{\infty}$ generated by finite-dimensional rectangles with components in $\mathcal{A}$, let $T$ be the left shift operator on $A^{\infty}$, and let $\mu$ be a measure on the measurable space $\left(A^{\infty}, \mathcal{A}^{\infty}\right)$, stationary with respect to $T$. The abbreviation $[A, \mu]$ describes the source in the development that follows.

Now for any fixed integer $L \geq 1$ and each $\ell \in\{1, \cdots, L\}$, where $L$ is the number of levels of resolution in our desired multiresolution source code, let $\rho_{(\ell)}\left(x_{1}, y_{1}\right)<\infty$ be a realvalued nonnegative distortion measure for $x_{1} \in A, y_{1} \in \hat{A}_{(\ell)}$, where $\hat{A}_{(\ell)}$ is the $\ell$ th of $L$ abstract reproduction alphabets. Assume that $\rho_{(\ell)}\left(x_{1}, y_{1}\right)$ is continuous in $x_{1}$ for each $y_{1} \in$ $\hat{A}_{(\ell)}$ and that there exists a reference letter $y_{1}^{*(\ell)} \in \hat{A}_{(\ell)}$ such that $E_{\mu} \rho_{(\ell)}\left(X_{1}, y_{1}^{*(\ell)}\right)=d^{*(\ell)}<\infty$. Define

$$
\rho_{(\ell)}\left(x^{N}, y^{N}\right)=\sum_{i=1}^{N} \rho_{(\ell)}\left(x_{i}, y_{i}\right) .
$$

Typically, the reproduction alphabets and distortion measures do not change across resolutions. That is, $\hat{A}_{(\ell)}=\hat{A}$ and $\rho_{(\ell)}\left(x_{1}, y_{1}\right)=\rho\left(x_{1}, y_{1}\right)$ for all $\ell \in\{1, \cdots, L\}$ and all $\left(x_{1}, y_{1}\right) \in A \times \hat{A}$. Given this observation and the desire for notational simplicity, the $(\ell)$ is dropped from $\hat{A}, y_{1}^{*}, d^{*}$, and $\rho$ in the remainder of this work. Note, however, that all of the results presented apply to the most general case where the $L$ reproduction alphabets and their corresponding reference letters and distortion measures differ.

Let $Q^{L, N}=\left(Q_{(1)}^{N}, \cdots, Q_{(L)}^{N}\right)$ be an $L$-resolution, fixedor variable-rate block quantizer with blocklength $N$. For any $\ell \in\{1, \cdots, L\}, Q_{(\ell)}^{N}$ maps $A^{N}$ onto some finite or countable set of codewords $\left\{y^{N}\right\}_{(\ell)}$ from $\hat{A}^{N}$. Together these codewords comprise a codebook $\mathcal{C}_{(\ell)}^{N}=\left\{\left(y^{N},\left|y^{N}\right|\right)\right\}_{(\ell)}$ in which each codeword $y^{N}$ has an associated fixed- or variable-length binary description with length denoted by $\left|y^{N}\right|$. (In a slight abuse of notation, $y^{N} \in \mathcal{C}_{(\ell)}^{N}$ and $Q_{(\ell)}^{N}\left(x^{N}\right)=y^{N}$ appear alternatively with $\left(y^{N},\left|y^{N}\right|\right) \in \mathcal{C}_{(\ell)}^{N}$ and $Q_{(\ell)}^{N}\left(x^{N}\right)=\left(y^{N},\left|y^{N}\right|\right)$. Notice, however, that there may exist multiple codewords, $y^{N}, z^{N} \in$ $\mathcal{C}_{(\ell)}^{N}$ such that $y^{N}=z^{N}$ but $\left|y^{N}\right| \neq\left|z^{N}\right|$. These duplicate codewords may be useful if the same description is given different description lengths depending on the associated lower resolution descriptions.) The description at the $(\ell-1)$ th resolution is embedded in the description at the $\ell$ th resolution. As a result, for any fixed $\left(z^{N},\left|z^{N}\right|\right) \in \mathcal{C}_{(\ell-1)}^{N}$, it is possible that only a subset of $\mathcal{C}_{(\ell)}^{N}$ will actually be available for describing those $x^{N}$ such that $Q_{(\ell-1)}^{N}\left(x^{N}\right)=\left(z^{N},\left|z^{N}\right|\right)$. (For notational purposes, assume a unique resolution-0 description to deal with the case where $\ell=1$.) Thus for each $\left(z^{N},\left|z^{N}\right|\right) \in$
$\mathcal{C}_{(\ell-1)}^{N}$, define

$\mathcal{C}_{(\ell)}^{N}\left(z^{N}\right)$

$$
=\left\{y^{N} \in \mathcal{C}_{(\ell)}^{N}:\left(Q_{(\ell-1)}^{N}\right)^{-1}\left(z^{N}\right) \cap\left(Q_{(\ell)}^{N}\right)^{-1}\left(y^{N}\right) \neq \emptyset\right\}
$$

where

$$
\left(Q_{(\ell)}^{N}\right)^{-1}\left(y^{N}\right)=\left\{x^{N}: Q_{(\ell)}^{N}\left(x^{N}\right)=y^{N}\right\} .
$$

For each $z^{N} \in \mathcal{C}_{(\ell-1)}^{N}$, the description lengths in $\mathcal{C}_{(\ell)}^{N}\left(z^{N}\right)$ must satisfy the Kraft inequality

$$
\sum_{y^{N} \in \mathcal{C}_{(\ell)}^{N}\left(z^{N}\right)} 2^{-\left|y^{N}\right|} \leq 1
$$

If $Q^{L, N}$ is a fixed-rate code, then for all $y^{N} \in \mathcal{C}_{(\ell)}^{N},\left|y^{N}\right|=c_{\ell}$ for some constant $c_{\ell}$ dependent on $\ell$. That is, each code in $Q^{L, N}$ uses a fixed rate in each resolution, but the rate may vary from resolution to resolution. Let $\mathcal{Q}^{\mathrm{fr}}(L, N)$ denote the class of dimension- $N$ fixed-rate codes with $L$ resolutions. Similarly, let $\mathcal{Q}^{\operatorname{vr}}(L, N)$ denote the class of dimension- $N$ variable-rate codes with $L$ resolutions.

Given any fixed- or variable-rate code $Q^{L, N} \in \mathcal{Q}^{\text {fr }}(L, N)$ or $Q^{L, N} \in \mathcal{Q}^{\mathrm{vr}}(L, N)$, the (instantaneous) rate vector associated with coding source vector $x^{N}$ with quantizer $Q^{L, N}$ is

$$
r\left(x^{N}, Q^{L, N}\right)=\left(\left|Q_{(1)}^{N}\left(x^{N}\right)\right|, \cdots,\left|Q_{(L)}^{N}\left(x^{N}\right)\right|\right)
$$

where $\boldsymbol{r}\left(x^{N}, Q^{L, N}\right)=\left(r_{1}, \cdots, r_{L}\right)$ implies that for each $\ell \in\{1, \cdots, L\}$, an embedded description of total length $\sum_{i=1}^{\ell} r_{i}$ bits is used in describing $x^{N}$ to resolution $\ell$ with $Q^{L, N}$. The expected rate in describing $N$ symbols from $\mu$ with code $Q^{L, N}$ is

$$
\begin{aligned}
R\left(\mu, Q^{L, N}\right) & =E_{\mu} \boldsymbol{r}\left(X^{N}, Q^{L, N}\right) \\
& =\left(E_{\mu}\left|Q_{(1)}^{N}\left(X^{N}\right)\right|, \cdots, E_{\mu}\left|Q_{(L)}^{N}\left(X^{N}\right)\right|\right) .
\end{aligned}
$$

Likewise, the (instantaneous) distortion vector associated with coding $x^{N}$ with code $Q^{L, N}$ is

$$
\boldsymbol{d}\left(x^{N}, Q^{L, N}\right)=\left(\rho\left(x^{N}, Q_{(1)}^{N}\left(x^{N}\right)\right), \cdots, \rho\left(x^{N}, Q_{(L)}^{N}\left(x^{N}\right)\right)\right)
$$

where $d\left(x^{N}, Q^{L, N}\right)=\left(d_{1}, \cdots, d_{L}\right)$ implies that the distortion associated with the $\ell$ th-resolution description of $x^{N}$ with code $Q^{L, N}$ is $d_{\ell}$. The expected distortion associated with code $Q^{L, n}$ is

$$
\begin{aligned}
& \boldsymbol{D}\left(\mu, Q^{L, N}\right) \\
& \quad=E_{\mu} d\left(X^{N}, Q^{L, N}\right) \\
& \quad=\left(E_{\mu} \rho\left(X^{N}, Q_{(1)}^{N}\left(X^{N}\right)\right), \cdots, E_{\mu} \rho\left(X^{N}, Q_{(L)}^{N}\left(X^{N}\right)\right)\right) .
\end{aligned}
$$

For any $k \geq 1$ let $P_{k} \subset \mathbb{R}^{k}$ be the nonnegative cone defined as

$P_{k}=\left\{x^{k} \in \mathbb{R}^{k}: x^{k}=\left(x_{1}, x_{2}, \cdots, x_{k}\right) ; x_{i} \geq 0,1 \leq i \leq k\right\}$.

For any $x^{k}, y^{k} \in \mathbb{R}^{k}$, the notations $x^{k} \geq y^{k}$ and $y^{k} \leq x^{k}$ specify that $x^{k}-y^{k} \in P_{k}$. Then for any integer $N>0$ and any $\mu$, the $L$-resolution fixed-rate achievable distortion-rate region equals

$$
\mathcal{R}^{\mathrm{fr}, L}(\mu)=\overline{\bigcup_{N} \mathcal{R}_{N}^{\mathrm{fr}, L}(\mu)} .
$$


Here $\mathcal{R}_{N}^{\mathrm{fr}, L}(\mu)$ is the associated $N$ th-order L-resolution fixedrate achievable distortion-rate region defined as

$$
\mathcal{R}_{N}^{\mathrm{fr}, L}(\mu)=\frac{\left\{\left(r^{L}, d^{L}\right): \exists Q^{L, N} \in \mathcal{Q}^{\mathrm{fr}}(L, N)\right.}{\text { s.t. } \left.\frac{1}{N}\left(\boldsymbol{R}\left(\mu, Q^{L, N}\right), \boldsymbol{D}\left(\mu, Q^{L, N}\right)\right) \leq\left(r^{L}, d^{L}\right)\right\}}
$$

and for any set $S \subseteq \mathbb{R}^{2 L}, \bar{S}$ denotes the closure of $S$ with respect to the Euclidean norm. Further, since it will be useful to compare fixed-rate codes of a particular rate $R^{L}$, define the $L$-resolution fixed-rate- $R^{L}$ achievable distortion region $\mathcal{D}^{\mathrm{fr}, L}\left(R^{L}, \mu\right)$ as

$$
\mathcal{D}^{\mathrm{fr}, L}\left(R^{L}, \mu\right)=\overline{\bigcup_{N} \mathcal{D}_{N}^{\mathrm{fr}, L}\left(R^{L}, \mu\right)} .
$$

Here $\mathcal{D}_{N}^{\mathrm{fr}, L}\left(R^{L}, \mu\right)$ is the associated $N$ th-order L-resolution fixed-rate- $R^{L}$ achievable distortion region, defined as

$$
\begin{aligned}
& \mathcal{D}_{N}^{\mathrm{fr}, L}\left(R^{L}, \mu\right) \\
& =\left\{d^{L}: \exists Q^{L, N} \in \mathcal{Q}^{\mathrm{fr}, R^{L}}(L, N) \text { s.t. } \frac{1}{N} \boldsymbol{D}\left(\mu, Q^{L, N}\right) \leq d^{L}\right\}
\end{aligned}
$$

where $\mathcal{Q}^{\mathrm{fr}, R^{L}}(L, N) \subseteq \mathcal{Q}^{\mathrm{fr}}(L, N)$ describes the class of fixedrate codes with rate vector strictly less than $R^{L}$ and is given by

$$
\begin{aligned}
\mathcal{Q}^{\mathrm{fr}, R^{L}} & (L, N) \\
& =\left\{Q^{L, N} \in \mathcal{Q}^{\mathrm{fr}}(L, N): \frac{1}{N} \boldsymbol{R}\left(\mu, Q^{L, N}\right)<R^{L}\right\} .
\end{aligned}
$$

Finally, for variable-rate coding the $L$-resolution variable-rate achievable distortion-rate region equals

$$
\mathcal{R}^{\mathrm{vr}, L}(\mu)=\overline{\bigcup_{N} \mathcal{R}_{N}^{\mathrm{vr}, L}(\mu)}
$$

where $\mathcal{R}_{N}^{\mathrm{vr}, L}(\mu)$ is the $N$ th-order L-resolution variable-rate achievable distortion-rate region defined as

$$
\mathcal{R}_{N}^{\mathrm{vr}, L}(\mu)=\frac{\overline{\left\{\left(r^{L}, d^{L}\right): \exists Q^{L, N} \in \mathcal{Q}^{\mathrm{vr}}(L, N)\right.}}{\text { s.t. } \left.\frac{1}{N}\left(R\left(\mu, Q^{L, N}\right), \boldsymbol{D}\left(\mu, Q^{L, N}\right)\right) \leq\left(r^{L}, d^{L}\right)\right\}} .
$$

Thus the $N$ th-order distortion-rate region describes the collection of rate and distortion pairs $\left(r^{L}, d^{L}\right)$ such that there exists an $N$-dimensional code with per-symbol rate and distortion less than or arbitrarily close (in Euclidean distance) to $\left(r^{L}, d^{L}\right)$. The achievable distortion-rate region is the set of $\left(r^{L}, d^{L}\right)$ points that can be approximated with arbitrary accuracy by points in the $N$ th-order distortion-rate regions. The goal of this work is to characterize the fixed- and variablerate achievable distortion-rate regions for a wide variety of source distributions $\mu$.

Derivation of some basic properties of $\mathcal{R}^{\mathrm{fr}, L}(\mu)$, $\mathcal{D}^{\mathrm{fr}, L}\left(R^{L}, \mu\right)$, and $\mathcal{R}^{\mathrm{vr}, L}(\mu)$ lends useful insight. The following two lemmas result from simple time-sharing arguments similar to those used for single-resolution source codes. The proofs for both of these results appear in the Appendix. The first result relies on the notion of a limit of sets. Given any sequence $\left\{S_{N}: N \geq 1\right\}$ of sets

$$
\limsup S_{N}=\bigcap_{M \geq 1} \bigcup_{N \geq M} S_{N}
$$

and

$$
\liminf S_{N}=\bigcup_{M \geq 1} \bigcap_{N \geq M} S_{N} .
$$

If $\limsup _{N} S_{N}=\liminf _{N} S_{N}$, then the $\operatorname{limit}_{\lim _{N}} S_{N}$ is defined as $\lim _{N} S_{N}=\limsup _{N} S_{N}=\liminf _{N} S_{N}$. Otherwise, the limit is undefined.

Lemma 1: If $\mu$ is a stationary source, then

$$
\begin{aligned}
& \mathcal{R}^{\mathrm{fr}, L}(\mu)=\overline{\lim _{N \rightarrow \infty} \mathcal{R}_{N}^{\mathrm{fr}, L}(\mu)} \\
& \mathcal{D}^{\mathrm{fr}, L}\left(R^{L}, \mu\right)=\overline{\lim _{N \rightarrow \infty} \mathcal{D}_{N}^{\mathrm{fr}, L}\left(R^{L}, \mu\right)} \\
& \mathcal{R}^{\mathrm{vr}, L}(\mu)=\overline{\lim _{N \rightarrow \infty} \mathcal{R}_{N}^{\mathrm{vr}, L}(\mu)} .
\end{aligned}
$$

Lemma 2: If $\mu$ is a stationary source, then $\mathcal{R}^{\mathrm{fr}, L}(\mu)$, $\mathcal{D}^{\mathrm{fr}, L}\left(R^{L}, \mu\right)$, and $\mathcal{R}^{\mathrm{vr}, L}(\mu)$ are convex sets.

Lemma 3 is a consequence of Lemma 2 that is helpful in better understanding $\mathcal{R}^{\mathrm{fr}, L}(\mu)$ and $\mathcal{R}^{\mathrm{vr}, L}(\mu)$. This result demonstrates that it does not matter how you divide up the rate between the resolutions of a multiresolution code as long as the total rate $R_{1}+R_{2}+\cdots+R_{\ell}$ for the $\ell$ th resolution is sufficiently high for each $\ell \in\{1, \cdots, L\}$. The proof of Lemma 3 is likewise left to the Appendix.

Lemma 3: If $\left(r^{L}, d^{L}\right) \in \mathcal{R}^{\mathrm{fr}, L}(\mu)$, then for any $R^{L} \geq \mathbf{0}$ such that

$\sum_{i=1}^{\ell} R_{i} \geq \sum_{i=1}^{\ell} r_{i}, \quad$ for all $1 \leq \ell \leq L,\left(R^{L}, d^{L}\right) \in \mathcal{R}^{\mathrm{fr}, L}(\mu)$.

Similarly, if $\left(r^{L}, d^{L}\right) \in \mathcal{R}^{\mathrm{vr}, L}(\mu)$, then for any $R^{L} \geq \mathbf{0}$ such that

$\sum_{i=1}^{\ell} R_{i} \geq \sum_{i=1}^{\ell} r_{i}, \quad$ for all $1 \leq \ell \leq L,\left(R^{L}, d^{L}\right) \in \mathcal{R}^{\mathrm{vr}, L}(\mu)$.

Lemma 2 and (1), (3), and (6) imply that for stationary $\mu$, $\mathcal{R}^{\mathrm{fr}, L}(\mu), \quad \mathcal{D}^{\mathrm{fr}, L}\left(R^{L}, \mu\right)$, and $\mathcal{R}^{\mathrm{vr}, L}(\mu)$ are convex and closed. As a result, each is entirely characterized by its support functional [20, p. 135], here given by $j^{\mathrm{fr}}\left(\alpha^{L}, \beta^{L}, \mu\right)$, $j^{\mathrm{fr}, R^{L}}\left(\alpha^{L}, \beta^{L}, \mu\right)$, and $j^{\mathrm{vr}}\left(\alpha^{L}, \beta^{L}, \mu\right)$, and called the weighted fixed-rate, fixed-rate- $R^{L}$, and variable-rate operational distortion-rate functions, respectively, where

$$
\begin{aligned}
j^{\mathrm{fr}}\left(\alpha^{L}, \beta^{L}, \mu\right) & =\inf _{\left(r^{L}, d^{L}\right) \in \mathcal{R}^{\mathrm{fr}, L}(\mu)} \sum_{\ell=1}^{L}\left(\alpha_{\ell} d_{\ell}+\beta_{\ell} r_{\ell}\right) \\
j^{\mathrm{fr}, R^{L}}\left(\alpha^{L}, \beta^{L}, \mu\right) & =\inf _{d^{L} \in \mathcal{D}^{\mathrm{fr}, L}\left(R^{L}, \mu\right)} \sum_{\ell=1}^{L}\left(\alpha_{\ell} d_{\ell}+\beta_{\ell} R_{\ell}\right) \\
j^{\mathrm{vr}}\left(\alpha^{L}, \beta^{L}, \mu\right) & =\inf _{\left(r^{L}, d^{L}\right) \in \mathcal{R}^{\mathrm{vr}, L}(\mu)} \sum_{\ell=1}^{L}\left(\alpha_{\ell} d_{\ell}+\beta_{\ell} r_{\ell}\right) .
\end{aligned}
$$


Notice first that

$$
j^{\mathrm{fr}}\left(\alpha^{L}, \beta^{L}, \mu\right)=\inf _{R^{L}} j^{\mathrm{fr}, R^{L}}\left(\alpha^{L}, \beta^{L}, \mu\right) .
$$

Notice further that the term $\sum_{\ell=1}^{L} \beta_{\ell} R_{\ell}$ in the definition of $j^{\mathrm{fr}, R^{L}}\left(\alpha^{L}, \beta^{L}, \mu\right)$ is a constant included only to make the three definitions parallel. In the equations for $j^{\mathrm{fr}}\left(\alpha^{L}, \beta^{L}, \mu\right)$ and $j^{\operatorname{vr}}\left(\alpha^{L}, \beta^{L}, \mu\right)$, the vector $\left(\alpha^{L}, \beta^{L}\right)$ may be interpreted as the planar direction or "slope" of the tangential hyperplane supporting the space of achievable $\left(R^{L}, D^{L}\right)$ vectors at a single point. The vector $\alpha^{L}$ plays a similar role in $j^{\mathrm{fr}, R^{L}}\left(\alpha^{L}, \beta^{L}, \mu\right)$.

The following properties of the weighted operational distortion-rate functions are useful for appropriately focusing a study of $j^{\mathrm{fr}}\left(\alpha^{L}, \beta^{L}, \mu\right), j^{\mathrm{fr}, R^{L}}\left(\alpha^{L}, \beta^{L}, \mu\right)$, and $j^{\operatorname{vr}}\left(\alpha^{L}, \beta^{L}, \mu\right)$.

Lemma 4: For any stationary source $\mu$ :

a) If $\alpha_{\ell}<0$ or $\beta_{\ell}<0$ for any $1 \leq \ell \leq L$, then

$$
j^{\mathrm{fr}}\left(\alpha^{L}, \beta^{L}, \mu\right)=j^{\mathrm{vr}}\left(\alpha^{L}, \beta^{L}, \mu\right)=-\infty .
$$

Similarly, if $\alpha_{\ell}<0$ for any $1 \leq \ell \leq L$, then

$$
j^{\mathrm{fr}, R^{L}}\left(\alpha^{L}, \beta^{L}, \mu\right)=-\infty \text {. }
$$

b) If $c \geq 0$, then

$$
\begin{gathered}
j^{\mathrm{fr}}\left(c \alpha^{L}, c \beta^{L}, \mu\right)=c j^{\mathrm{fr}}\left(\alpha^{L}, \beta^{L}, \mu\right), \\
j^{\mathrm{fr}, R^{L}\left(c \alpha^{L}, c \beta^{L}, \mu\right)}=c j^{\mathrm{fr}, R^{L}}\left(\alpha^{L}, \beta^{L}, \mu\right),
\end{gathered}
$$

and

$$
j^{\mathrm{vr}}\left(c \alpha^{L}, c \beta^{L}, \mu\right)=c j^{\mathrm{vr}}\left(\alpha^{L}, \beta^{L}, \mu\right) .
$$

Proof: In proving the first result, notice that for any stationary $\mu$, the existence of a reference letter implies

$$
\left(\boldsymbol{0}, \boldsymbol{d}^{*}\right)=\left((0, \cdots, 0),\left(d^{*}, \cdots, d^{*}\right)\right) \in \mathcal{R}^{\mathrm{fr}, L}(\mu) \subseteq \mathcal{R}^{\mathrm{vr}, L}(\mu)
$$

for all $\mu$ and

$$
\left(d^{*}, \cdots, d^{*}\right) \in \mathcal{D}^{\mathrm{fr}, L}\left(R^{L}, \mu\right)
$$

for all $R^{L} \geq \mathbf{0}$ and all $\mu$. Thus for all $\left(r^{L}, d^{L}\right) \geq\left(\mathbf{0}, \boldsymbol{d}^{*}\right)$

$$
\left(r^{L}, d^{L}\right) \in \mathcal{R}^{\mathrm{fr}, L}(\mu) \subseteq \mathcal{R}^{\mathrm{vr}, L}(\mu)
$$

and $d^{L} \in \mathcal{D}^{\mathrm{fr}, L}\left(R^{L}, \mu\right)$, giving the first result. The second result is immediate.

As a result of Lemma 4 a), there is no loss of generality in restricting our attention to $\alpha^{L}, \beta^{L}$ such that $\left(\alpha^{L}, \beta^{L}\right) \geq \mathbf{0}$. Further, by Lemma $4 \mathrm{~b}$ ), it is the relative rather than the absolute sizes of the $\alpha$ and $\beta$ values that determine the behavior of $j^{\mathrm{fr}}\left(\alpha^{L}, \beta^{L}, \mu\right), j^{\mathrm{fr}, R^{L}}\left(\alpha^{L}, \beta^{L}, \mu\right)$, and $j^{\mathrm{vr}}\left(\alpha^{L}, \beta^{L}, \mu\right)$. Thus a further restriction to $\alpha^{L}, \beta^{L} \geq \mathbf{0}$ such that

$$
\sum_{\ell=1}^{L}\left(\alpha_{\ell}+\beta_{\ell}\right)=1
$$

simplifies the characterization of $j^{\mathrm{fr}}\left(\alpha^{L}, \beta^{L}, \mu\right)$, $j^{\mathrm{fr}, R^{L}}\left(\alpha^{L}, \beta^{L}, \mu\right)$, and $j^{\mathrm{vr}}\left(\alpha^{L}, \beta^{L}, \mu\right)$ with no loss of generality.
A rate distortion pair $\left(r^{L}, d^{L}\right)$ is achievable by a fixed-rate code if $\left(r^{L}, d^{L}\right) \in \mathcal{R}^{\mathrm{fr}, L}(\mu)$ or, equivalently, if

$$
\sum_{\ell=1}^{L}\left(\alpha_{\ell} d_{\ell}+\beta_{\ell} r_{\ell}\right) \geq j^{\mathrm{fr}}\left(\alpha^{L}, \beta^{L}, \mu\right)
$$

for all $\alpha^{L}, \beta^{L} \geq \mathbf{0}$ such that $\sum_{\ell=1}^{L}\left(\alpha_{\ell}+\beta_{\ell}\right)=1$, and achievable by a variable-rate code if $\left(r^{L}, d^{L}\right) \in \mathcal{R}^{\mathrm{vr}, L}(\mu)$ or, equivalently, if

$$
\sum_{\ell=1}^{L}\left(\alpha_{\ell} d_{\ell}+\beta_{\ell} r_{\ell}\right) \geq j^{\mathrm{vr}}\left(\alpha^{L}, \beta^{L}, \mu\right)
$$

for all $\alpha^{L}, \beta^{L} \geq \mathbf{0}$ such that $\sum_{\ell=1}^{L}\left(\alpha_{\ell}+\beta_{\ell}\right)=1$. A distortion vector $d^{L}$ is achievable by a fixed-rate- $R^{L}$ code if $d^{L} \in \mathcal{D}^{\mathrm{fr}, L}\left(R^{L}, \mu\right)$ or equivalently if for any $\beta^{L} \geq \mathbf{0}$

$$
\sum_{\ell=1}^{L}\left(\alpha_{\ell} d_{\ell}+\beta_{\ell} R_{\ell}\right) \geq j^{\mathrm{fr}, R^{L}}\left(\alpha^{L}, \beta^{L}, \mu\right)
$$

for all $\alpha^{L} \geq \mathbf{0}$ such that $\sum_{\ell=1}^{L} \alpha_{\ell}=1$.

The following lemma establishes an alternative pair of definitions for the operational distortion-rate functions $j^{\mathrm{fr}}\left(\alpha^{L}, \beta^{L}, \mu\right), \quad j^{\mathrm{fr}, R^{L}}\left(\alpha^{L}, \beta^{L}, \mu\right), \quad$ and $j^{\mathrm{vr}}\left(\alpha^{L}, \beta^{L}, \mu\right)$ to the definitions given by (8)-(10). In particular, while $j^{\mathrm{fr}}\left(\alpha^{L}, \beta^{L}, \mu\right), j^{\mathrm{fr}, R^{L}}\left(\alpha^{L}, \beta^{L}, \mu\right)$, and $j^{\mathrm{vr}}\left(\alpha^{L}, \beta^{L}, \mu\right)$ were originally specified as the support functionals for the fixedrate, fixed-rate- $R^{L}$, and variable-rate achievable distortionrate regions, they can also be described in terms of the corresponding $N$ th-order weighted operational distortion-rate functions.

Lemma 5: Let $\quad j^{\mathrm{fr}}\left(\alpha^{L}, \beta^{L}, \mu\right), \quad j^{\mathrm{fr}, R^{L}}\left(\alpha^{L}, \beta^{L}, \mu\right), \quad$ and $j^{\mathrm{vr}}\left(\alpha^{L}, \beta^{L}, \mu\right)$ be as defined in (8)-(10). Then

$$
\begin{gathered}
j^{\mathrm{fr}}\left(\alpha^{L}, \beta^{L}, \mu\right)=\inf _{N} j_{N}^{\mathrm{fr}}\left(\alpha^{L}, \beta^{L}, \mu\right) \\
j^{\mathrm{fr}, R^{L}}\left(\alpha^{L}, \beta^{L}, \mu\right)=\inf _{N} j_{N}^{\mathrm{fr}, R^{L}}\left(\alpha^{L}, \beta^{L}, \mu\right) \\
j^{\mathrm{vr}}\left(\alpha^{L}, \beta^{L}, \mu\right)=\inf _{N} j_{N}^{\mathrm{vr}}\left(\alpha^{L}, \beta^{L}, \mu\right)
\end{gathered}
$$

where

$$
\begin{aligned}
j_{N}^{\mathrm{fr}}\left(\alpha^{L}, \beta^{L}, \mu\right)= & \frac{1}{N} \inf _{Q^{L, N} \in \mathcal{Q}^{\mathrm{fr}}(L, N)}\left(\alpha^{L} \boldsymbol{D}^{t}\left(\mu, Q^{L, N}\right)\right. \\
& \left.+\beta^{L} \boldsymbol{R}^{t}\left(\mu, Q^{L, N}\right)\right) \\
j_{N}^{\mathrm{fr}, R^{L}}\left(\alpha^{L}, \beta^{L}, \mu\right)= & \frac{1}{N} \inf _{Q^{L, N} \in \mathcal{Q}^{\mathrm{fr}, R^{L}}(L, N)}\left(\alpha^{L} \boldsymbol{D}^{t}\left(\mu, Q^{L, N}\right)\right. \\
& \left.+\beta^{L}\left(N R^{L}\right)^{t}\right) \\
j_{N}^{\mathrm{vr}}\left(\alpha^{L}, \beta^{L}, \mu\right)= & \frac{1}{N} \inf _{Q^{L, N} \in \mathcal{Q}^{\mathrm{vr}}(L, N)}\left(\alpha^{L} \boldsymbol{D}^{t}\left(\mu, Q^{L, N}\right)\right. \\
& \left.+\beta^{L} \boldsymbol{R}^{t}\left(\mu, Q^{L, N}\right)\right)
\end{aligned}
$$

and superscript $t$ denotes the transpose operator. 
Proof: The three proofs are almost identical, and thus only the proof for $j^{\mathrm{fr}}\left(\alpha^{L}, \beta^{L}, \mu\right)$ is shown. That proof contains two parts. The first part shows that

$$
j^{\mathrm{fr}}\left(\alpha^{L}, \beta^{L}, \mu\right) \geq \inf _{N} j_{N}^{\mathrm{fr}}\left(\alpha^{L}, \beta^{L}, \mu\right) .
$$

The second part shows that

$$
j^{\mathrm{fr}}\left(\alpha^{L}, \beta^{L}, \mu\right) \leq \inf _{N} j_{N}^{\mathrm{fr}}\left(\alpha^{L}, \beta^{L}, \mu\right) .
$$

Using definition (8), for any $\epsilon>0$, there exists $\left(r^{L}, d^{L}\right) \epsilon$ $\mathcal{R}^{\mathrm{fr}, L}(\mu)$ such that

$$
j^{\mathrm{fr}}\left(\alpha^{L}, \beta^{L}, \mu\right) \geq \sum_{\ell=1}^{L}\left(\alpha_{\ell} d_{\ell}+\beta_{\ell} r_{\ell}\right)-\epsilon .
$$

Further, by (1) and (2), there exist $N \geq 1$ and $Q^{L, N} \in$ $\mathcal{Q}^{\mathrm{fr}}(L, N)$ such that

$$
\left(r^{L}, d^{L}\right) \geq(1 / N)\left(\boldsymbol{R}\left(\mu, Q^{L, N}\right), \boldsymbol{D}\left(\mu, Q^{L, N}\right)\right)-(\epsilon, \cdots, \epsilon) .
$$

Thus there exist a dimension $N$ and quantizer $Q^{L, N} \in$ $\mathcal{Q}^{\mathrm{fr}}(L, N)$ such that

$$
\begin{aligned}
f^{\mathrm{fr}}\left(\alpha^{L}, \beta^{L}, \mu\right) \geq & (1 / N)\left(\alpha^{L} \boldsymbol{D}^{t}\left(\mu, Q^{L, N}\right)\right. \\
& \left.+\beta^{L} \boldsymbol{R}^{t}\left(\mu, Q^{L, N}\right)\right)-2 \epsilon .
\end{aligned}
$$

Since $\epsilon$ is arbitrary and the above property holds for a particular $N \geq 1$ and $Q^{L, N} \in \mathcal{Q}^{\mathrm{fr}}(L, N)$

$$
j^{\mathrm{fr}}\left(\alpha^{L}, \beta^{L}, \mu\right) \geq \inf _{N} j_{N}^{\mathrm{fr}}\left(\alpha^{L}, \beta^{L}, \mu\right) .
$$

On the other hand, for any $\epsilon>0$, there exist a dimension $N \geq 1$ and a code $Q^{L, N} \in \mathcal{Q}^{\mathrm{fr}}(L, N)$ such that

$$
\begin{aligned}
(1 / N)\left(\alpha^{L} \boldsymbol{D}^{t}\left(\mu, Q^{L, N}\right)+\beta^{L} \boldsymbol{R}^{t}(\mu,\right. & \left.\left.Q^{L, N}\right)\right) \\
& \leq \inf _{N} j_{N}^{\mathrm{fr}}\left(\alpha^{L}, \beta^{L}, \mu\right)+\epsilon .
\end{aligned}
$$

Thus

$$
\begin{aligned}
j^{\mathrm{fr}}\left(\alpha^{L}, \beta^{L}, \mu\right) \leq & (1 / N)\left(\alpha^{L} \boldsymbol{D}^{t}\left(\mu, Q^{L, N}\right)\right. \\
& \left.+\beta^{L} \boldsymbol{R}^{t}\left(\mu, Q^{L, N}\right)\right)
\end{aligned}
$$

implies

$$
j^{\mathrm{fr}}\left(\alpha^{L}, \beta^{L}, \mu\right) \leq \inf _{N} j_{N}^{\mathrm{fr}}\left(\alpha^{L}, \beta^{L}, \mu\right)+\epsilon .
$$

Once again, since $\epsilon$ is arbitrary the desired result follows.

A number of information-theoretic quantities arise in characterizing the weighted operational distortion-rate functions $j^{\mathrm{fr}}\left(\alpha^{L}, \beta^{L}, \mu\right), j^{\mathrm{fr}, R^{L}}\left(\alpha^{L}, \beta^{L}, \mu\right)$, and $j^{\mathrm{vr}}\left(\alpha^{L}, \beta^{L}, \mu\right)$. Their definitions follow. Let $q^{N}$ be any conditional probability measure or test channel from $A^{N}$ to $\left(\hat{A}^{N}\right)^{L}$ defining, with $\mu^{N}$, a joint probability $p^{N}=\mu^{N} q^{N}$ on

$$
A^{N} \times\left(\hat{A}^{N}\right)^{L}=A^{N} \times \hat{A}^{N} \times \cdots \times \hat{A}^{N} .
$$

Use $\nu^{N}$ to denote the associated marginal on $\left(\hat{A}^{N}\right)^{L}$ and $\pi^{N}$ to denote the product probability induced by the marginals $\mu^{N}$ and $\nu^{N}$. If $p^{N} \ll \pi^{N}$, then the Radon-Nikodym (R-N) theorem implies the existence of the R-N derivative $f_{N}=$ $d p^{N} / d \pi^{N}$, which is unique $\pi^{N}$-almost everywhere and measurable with respect to $\mathcal{A}^{N} \times\left(\hat{\mathcal{A}}^{N}\right)^{L}$ [5], where $\hat{\mathcal{A}}^{N}$ is the $\sigma$-algebra of $\hat{A}^{N}$. For any $1 \leq \ell \leq L$, the resulting average mutual information may be expressed (e.g., [5, Theorem 7.1.3]) as

$$
I_{p^{N}}\left(X^{N} ; \boldsymbol{Y}^{\ell}\right)=\int \log f_{N}\left(x^{N} ; \boldsymbol{y}^{\ell}\right) d p^{N}\left(x^{N}, \boldsymbol{y}^{\ell}\right)
$$

where $\boldsymbol{Y}^{\ell}=\left(\boldsymbol{Y}_{1}, \boldsymbol{Y}_{2}, \cdots, \boldsymbol{Y}_{\ell}\right)$ is a random vector representing the first $\ell$ reproductions of $X^{N}$ and $y^{\ell}$ is a particular instance of $\boldsymbol{Y}_{\ell}$. (The boldface type here denotes that $\boldsymbol{Y}_{\ell}$ and $\boldsymbol{y}_{\ell}$ are reproduction vectors rather than scalar quantities. The dimension of the reproduction $\boldsymbol{y}_{\ell}$ must be identical to the dimension of the original data vector, and thus may be determined from the context of $\boldsymbol{y}_{\ell}$. Thus in the above equation, $\boldsymbol{y}_{\ell} \in \hat{A}^{N}$.) Likewise, define the mutual information rate $I_{p}\left(\mathcal{X} ; \mathcal{Y}^{\ell}\right)$ as

$$
I_{p}\left(\mathcal{X} ; \mathcal{Y}^{\ell}\right)=\lim _{N \rightarrow \infty} \frac{1}{N} I_{p^{N}}\left(X^{N} ; \boldsymbol{Y}^{\ell}\right) .
$$

Let $J\left(\alpha^{L}, \beta^{L}, \mu\right)$, here called the weighted distortion-rate function, be defined as

$$
J\left(\alpha^{L}, \beta^{L}, \mu\right)=\inf _{N} J_{N}\left(\alpha^{L}, \beta^{L}, \mu\right)
$$

where the Nth-order weighted distortion-rate function $J_{N}\left(\alpha^{L}, \beta^{L}, \mu\right)$ is defined as

$$
\begin{aligned}
J_{N}\left(\alpha^{L}, \beta^{L}, \mu\right)= & \inf _{q} \frac{1}{N} \sum_{\ell=1}^{L}\left(\alpha_{\ell} E_{\mu^{N} q^{N}} \rho\left(X^{N}, \boldsymbol{Y}_{\ell}\right)\right. \\
& \left.+\beta_{\ell} I_{\mu^{N} q^{N}}\left(X^{N} ; \boldsymbol{Y}_{\ell} \mid \boldsymbol{Y}^{\ell-1}\right)\right)
\end{aligned}
$$

and $I_{\mu^{N} q^{N}}\left(X^{N} ; \boldsymbol{Y}_{\ell} \mid \boldsymbol{Y}^{\ell-1}\right)$ is the conditional mutual information, which may be described as

$$
\begin{aligned}
I_{\mu^{N} q^{N}}\left(X^{N} ; \boldsymbol{Y}_{\ell} \mid \boldsymbol{Y}^{\ell-1}\right)= & I_{\mu^{N} q^{N}}\left(X^{N} ; \boldsymbol{Y}^{\ell}\right) \\
& -I_{\mu^{N} q^{N}}\left(X^{N} ; \boldsymbol{Y}^{\ell-1}\right)
\end{aligned}
$$

if both terms in the difference are finite. Similarly, let $J^{R^{L}}\left(\alpha^{L}, \beta^{L}, \mu\right)$, here called the rate- $R^{L}$ weighted distortionrate function, be defined as

$$
J^{R^{L}}\left(\alpha^{L}, \beta^{L}, \mu\right)=\inf _{N} J_{N}^{R^{L}}\left(\alpha^{L}, \beta^{L}, \mu\right)
$$

where the $N$ th-order rate- $R^{L}$ weighted distortion-rate function $J_{N}^{R^{L}}\left(\alpha^{L}, \beta^{L}, \mu\right)$ is defined as

$$
\begin{aligned}
& J_{N}^{R^{L}}\left(\alpha^{L}, \beta^{L}, \mu\right) \\
&=\inf _{q}\left\{\frac{1}{N} \sum_{\ell=1}^{L}\left(\alpha_{\ell} E_{\mu^{N} q^{N}} \rho\left(X^{N}, \boldsymbol{Y}_{\ell}\right)+\beta_{\ell} N R_{\ell}\right):\right. \\
& \\
&\left.\quad \frac{1}{N} I_{\mu^{N} q^{N}}\left(X^{N} ; \boldsymbol{Y}_{\ell} \mid \boldsymbol{Y}^{\ell-1}\right)<R_{\ell} \forall \ell \in\{1, \cdots, L\}\right\} .
\end{aligned}
$$

Two immediate consequences of (15)-(18) are that

$$
J\left(\alpha^{L}, \beta^{L}, \mu\right)=\inf _{R^{L}} J^{R^{L}}\left(\alpha^{L}, \beta^{L}, \mu\right)
$$

and

$$
J_{N}\left(\alpha^{L}, \beta^{L}, \mu\right)=\inf _{R^{L}} J_{N}^{R^{L}}\left(\alpha^{L}, \beta^{L}, \mu\right)
$$

for all $N \geq 1$. 
Lemma 6 gives an alternative characterization of $J\left(\alpha^{L}, \beta^{L}, \mu\right)$ and $J^{R^{L}}\left(\alpha^{L}, \beta^{L}, \mu\right)$. Proof of Lemma 6 relies on the fact that if a nonnegative sequence $\left\{a_{N}: N=1,2, \cdots\right\}$ is subadditive, i.e., if $a_{N+k} \leq a_{N}+a_{k}$, then

$$
\lim _{N \rightarrow \infty} a_{N} / N=\inf _{N \geq 1} a_{N} / N
$$

[21, Lemma 7.5.1].

Lemma 6: If $\mu$ is stationary, then

$$
J\left(\alpha^{L}, \beta^{L}, \mu\right)=\lim _{N \rightarrow \infty} J_{N}\left(\alpha^{L}, \beta^{L}, \mu\right)
$$

and

$$
J^{R^{L}}\left(\alpha^{L}, \beta^{L}, \mu\right)=\lim _{N \rightarrow \infty} J_{N}^{R^{L}}\left(\alpha^{L}, \beta^{L}, \mu\right) .
$$

Proof: Fix $N, k \geq 1$ and $\epsilon>0$. By definition (16), there exist test channels $q^{N}$ and $\phi^{k}$ such that

$$
\begin{array}{r}
(1 / N) \sum_{\ell=1}^{L}\left(\alpha_{\ell} E_{\mu^{N} q^{N}} \rho\left(X^{N}, \boldsymbol{Y}_{\ell}\right)+\beta_{\ell} I_{\mu^{N} q^{N}}\left(X^{N} ; \boldsymbol{Y}_{\ell} \mid \boldsymbol{Y}^{\ell-1}\right)\right) \\
\leq J_{N}\left(\alpha^{L}, \beta^{L}, \mu\right)+\epsilon
\end{array}
$$

and

$$
\begin{array}{r}
(1 / k) \sum_{\ell=1}^{L}\left(\alpha_{\ell} E_{\mu^{k} \phi^{k}} \rho\left(X^{k}, \boldsymbol{Y}_{\ell}\right)+\beta_{\ell} I_{\mu^{k} \phi^{k}}\left(X^{k} ; \boldsymbol{Y}_{\ell} \mid \boldsymbol{Y}^{\ell-1}\right)\right) \\
\leq J_{k}\left(\alpha^{L}, \beta^{L}, \mu\right)+\epsilon .
\end{array}
$$

Let $q^{N+k}$ be the test channel equivalent to sending the first $N$ symbols of $X^{N+k}$ through test channel $q^{N}$ and the remaining $k$ symbols of $X^{N+k}$ through test channel $\phi^{k}$. That is, define a regular conditional probability $q^{N+k}\left(\cdot \mid x^{N+k}\right)$ by its values on rectangles as

$$
q^{N+k}\left(F \times G \mid x^{N+k}\right)=q^{N}\left(F \mid x^{N}\right) \phi^{k}\left(G \mid x_{N+1}^{N+k}\right)
$$

for each $F \in\left(\hat{\mathcal{A}}^{N}\right)^{L}$ and $G \in\left(\hat{\mathcal{A}}^{k}\right)^{L}$. Then, by stationarity and the conditional independence of the first $N$ channel outputs and the last $k$ channel outputs given the corresponding channel inputs

$$
\begin{aligned}
& I_{\boldsymbol{\mu}^{N+k} q^{N+k}}\left(X^{N+k} ; \boldsymbol{Y}_{\ell} \mid \boldsymbol{Y}^{\ell-1}\right) \\
& \quad \leq I_{\mu^{N} q^{N}}\left(X^{N} ; \boldsymbol{Y}_{\ell} \mid \boldsymbol{Y}^{\ell-1}\right)+I_{\mu^{k} \phi^{k}}\left(X^{k} ; \boldsymbol{Y}_{\ell} \mid \boldsymbol{Y}^{\ell-1}\right)
\end{aligned}
$$

for each $1 \leq \ell \leq L$. Further, by the stationarity of $\mu$

$$
\begin{aligned}
& E_{\mu^{N+k} q^{N+k}} \rho\left(X^{N+k}, \boldsymbol{Y}_{\ell}\right) \\
& \quad=E_{\mu^{N} q^{N}} \rho\left(X^{N}, \boldsymbol{Y}_{\ell}\right)+E_{\mu^{k} \phi^{k}} \rho\left(X^{k}, Y_{\ell}\right) .
\end{aligned}
$$

Thus

$$
\begin{aligned}
& (N+k) J_{N+k}\left(\alpha^{L}, \beta^{L}, \mu\right) \\
& \quad \leq N J_{N}\left(\alpha^{L}, \beta^{L}, \mu\right)+k J_{k}\left(\alpha^{L}, \beta^{L}, \mu\right)+(N+k) \epsilon
\end{aligned}
$$

for arbitrary $\epsilon$. That is, $N J_{N}$ is a subadditive sequence, giving the desired result by the equivalence of limits and infima for normalized subadditive sequences. The proof for $J^{R^{L}}\left(\alpha^{L}, \beta^{L}, \mu\right)$ is almost identical.

\section{RESULTS}

The main results of this paper are summarized below. In all cases assume $\alpha^{L}, \beta^{L} \geq \mathbf{0}$ and $\sum_{\ell=1}^{L}\left(\alpha_{\ell}+\beta_{\ell}\right)=1$.

\section{A. Stationary Ergodic Sources}

If $\mu$ is stationary and ergodic, $A$ is Polish, $\rho(x, y)$ is continuous in $x$ for each $y$, and there exists a reference letter $y_{1}^{*}$ such that $E_{\mu} \rho\left(X, y_{1}^{*}\right)<\infty$, then the following results hold.

Theorem 1: $j^{\mathrm{fr}, R^{L}}\left(\alpha^{L}, \beta^{L}, \mu\right)=J^{R^{L}}\left(\alpha^{L}, \beta^{L}, \mu\right)$.

Theorem 2: $j^{\mathrm{fr}}\left(\alpha^{L}, \beta^{L}, \mu\right)=J\left(\alpha^{L}, \beta^{L}, \mu\right)$.

Theorem 3: $j^{\operatorname{vr}}\left(\alpha^{L}, \beta^{L}, \mu\right)=J\left(\alpha^{L}, \beta^{L}, \mu\right)$.

Theorem 4: $\mathcal{R}^{\mathrm{fr}, L}(\mu)=\mathcal{R}^{\mathrm{vr}, L}(\mu)$.

\section{B. Stationary Nonergodic Sources}

When $\mu$ is stationary and nonergodic, let $\left\{\mu_{x}: x \in A^{\infty}\right\}$ denote the ergodic decomposition of $\mu$. The ergodic decomposition exists since if $A$ is Polish, then $(A, \mathcal{A})$ is standard [21, Lemma 3.3.1], and hence $\left(A^{\infty}, \mathcal{A}^{\infty}\right)$ is standard [21, Lemma 2.4.1]; standard measurable spaces admit the ergodic decomposition [21, Theorem 7.4.1]. Under the conditions given above (namely $A$ is Polish, $\rho(x, y)$ is continuous in $x$ for each $y$, and there exists a reference letter $y_{1}^{*}$ such that $\left.E_{\mu} \rho\left(X, y_{1}^{*}\right)<\infty\right)$, the following results hold.

\section{Theorem 5:}

$$
j^{\mathrm{fr}, R^{L}}\left(\alpha^{L}, \beta^{L}, \mu\right)=\int j^{\mathrm{fr}, R^{L}}\left(\alpha^{L}, \beta^{L}, \mu_{x}\right) d \mu(x) .
$$

Theorem 6:

$$
j^{\mathrm{fr}}\left(\alpha^{L}, \beta^{L}, \mu\right)=\inf _{R^{L}} \int j^{\mathrm{fr}, R^{L}}\left(\alpha^{L}, \beta^{L}, \mu_{x}\right) d \mu(x) .
$$

Theorem 7: $j^{\mathrm{vr}}\left(\alpha^{L}, \beta^{L}, \mu\right)=\int j^{\mathrm{vr}}\left(\alpha^{L}, \beta^{L}, \mu_{x}\right) d \mu(x)$.

Theorem 8: $J\left(\alpha^{L}, \beta^{L}, \mu\right)=\int J\left(\alpha^{L}, \beta^{L}, \mu_{x}\right) d \mu(x)$.

Theorem 9:

$$
j^{\mathrm{fr}, R^{L}}\left(\alpha^{L}, \beta^{L}, \mu\right)=\int J^{R^{L}}\left(\alpha^{L}, \beta^{L}, \mu_{x}\right) d \mu(x) .
$$

Theorem 10: $j^{\mathrm{fr}}\left(\alpha^{L}, \beta^{L}, \mu\right) \geq J\left(\alpha^{L}, \beta^{L}, \mu\right)$.

Theorem 11: $j^{\mathrm{vr}}\left(\alpha^{L}, \beta^{L}, \mu\right)=J\left(\alpha^{L}, \beta^{L}, \mu\right)$.

Theorem 12: $\mathcal{R}^{\mathrm{fr}, L}(\mu) \subseteq \mathcal{R}^{\mathrm{vr}, L}(\mu)$.

Theorem 2 is an immediate consequence of Theorem 1, (11), and (19). Theorem 4 follows from Theorems 2 and 3. Theorem 6 combines Theorem 5 and (11). Theorem 9 combines Theorems 5 and 1. Combining this result with (11), (19), and Theorem 8 immediately yields Theorem 10. Note that equality holds in Theorem 10 if and only if

$\inf _{R^{L}} \int J^{R^{L}}\left(\alpha^{L}, \beta^{L}, \mu_{x}\right) d \mu(x)=\int \inf _{R^{L}} J^{R^{L}}\left(\alpha^{L}, \beta^{L}, \mu_{x}\right) d \mu(x)$. 
Thus fixed-rate multiresolution codes achieve the optimal performance only when the ergodic modes of the stationary nonergodic source $\mu$ almost surely have the same weighted distortion-rate curves. Theorem 11 results immediately from Theorems 3, 7, and 8. Theorem 12 is a consequence of Theorems 10 and 11. The implications of these results are discussed in Section IV. The proofs of Theorems 1, 3, 5, 7, and 8 are given in a series of lemmas in Section VI.

\section{DISCUSSION}

Examination of Theorems 1-12 from a variety of perspectives yields greater insight into their meaning and how they relate to earlier results.

First, it should be noted that $L=1$ corresponds to singleresolution source coding, which is the traditional source coding problem. For $L=1$, Theorems 1-12 teach us that the weighted distortion-rate function lower-bounds the operational weighted distortion-rate function for stationary sources on Polish alphabets. Further, the weighted distortion-rate function of a stationary ergodic source is achievable by both fixed- and variable-rate source codes. The weighted distortion-rate function of a stationary nonergodic source is, roughly speaking, the expected value of the corresponding weighted distortion-rate functions of its subsources, where the expectations are taken over points of equal slope. Further, this weighted distortionrate function is achievable only by variable-rate source codes (or mixtures of fixed-rate codes at varying rates) unless almost all of the ergodic modes of the source have the same weighted distortion-rate function.

Performance bounds are known for both stationary ergodic and stationary nonergodic sources on Polish alphabets. The following argument verifies the consistency of the above-described theorems with earlier results. Let the operational distortion-rate functions $\delta^{\mathrm{fr}}(R, \mu)$ and $\delta^{\mathrm{vr}}(R, \mu)$ and the distortion-rate function $D(R, \mu)$ be defined as

$$
\begin{aligned}
\delta^{\mathrm{fr}}(R, \mu) & =\inf _{N} \delta_{N}^{\mathrm{fr}}(R, \mu) \\
\delta^{\mathrm{vr}}(R, \mu) & =\inf _{N} \delta_{N}^{\mathrm{vr}}(R, \mu) \\
D(R, \mu) & =\inf _{N} D_{N}(R, \mu)
\end{aligned}
$$

where

$$
\begin{aligned}
& \delta_{N}^{\mathrm{fr}}(R, \mu)=\inf _{Q^{1, N} \in \mathcal{Q}^{\mathrm{fr}}(1, N)}\{ \frac{1}{N} \boldsymbol{D}\left(\mu, Q^{1, N}\right): \\
&\left.\frac{1}{N} \boldsymbol{R}\left(\mu, Q^{1, N}\right) \leq R\right\} \\
& \delta_{N}^{\operatorname{vr}}(R, \mu)=\inf _{Q^{1, N} \in \mathcal{Q}^{\operatorname{vr}}(1, N)}\left\{\frac{1}{N} \boldsymbol{D}\left(\mu, Q^{1, N}\right):\right. \\
&\left.\frac{1}{N} \boldsymbol{R}\left(\mu, Q^{1, N}\right) \leq R\right\} \\
& D_{N}(R, \mu)=\inf _{q}\left\{\frac{1}{N} E_{\mu^{N} q^{N}} \rho\left(X^{N}, Y^{N}\right):\right. \\
&\left.\frac{1}{N} I_{\mu^{N} q^{N}}\left(X^{N} ; Y^{N}\right) \leq R\right\} .
\end{aligned}
$$

Then by the Lagrange duality theorem [20, p. 224]

$$
\begin{array}{cc}
\max _{\alpha, \beta \geq 0} \frac{1}{\alpha}\left(j^{\mathrm{fr}}(\alpha, \beta, \mu)-\beta R_{1}\right) & \\
=\inf _{N} \inf _{Q^{1, N} \in \mathcal{Q}^{\mathrm{fr}}(1, N)}\left\{\frac{1}{N} D\left(\mu, Q^{1, N}\right):\right. & \left.\frac{1}{N} R\left(\mu, Q^{1, N}\right) \leq R_{1}\right\} \\
=\delta^{\mathrm{fr}}\left(R_{1}, \mu\right), & \\
\max _{\alpha, \beta \geq 0} \frac{1}{\alpha}\left(j^{\mathrm{vr}}(\alpha, \beta, \mu)-\beta R_{1}\right) & \\
=\inf _{N} \inf _{Q^{1, N} \in \mathcal{Q}^{\mathrm{vr}}(1, N)}\left\{\frac{1}{N} D\left(\mu, Q^{1, N}\right):\right. \\
=\delta^{\mathrm{vr}}\left(R_{1}, \mu\right),
\end{array}
$$

and

$$
\begin{aligned}
& \max _{\alpha, \beta \geq 0} \frac{1}{\alpha}\left(J(\alpha, \beta, \mu)-\beta R_{1}\right) \\
&=\inf _{N} \inf _{q}\left\{\frac{1}{N} E_{\mu^{N} q^{N}} \rho\left(X^{N}, \boldsymbol{Y}_{1}\right):\right. \\
&\left.\quad \frac{1}{N} I_{\mu^{N} q^{N}}\left(X^{N} ; \boldsymbol{Y}_{1}\right) \leq R_{1}\right\} \\
&=D\left(R_{1}, \mu\right) .
\end{aligned}
$$

The Lagrange duality theorem also lends insight into the integrals $\int j^{\mathrm{fr}}\left(\alpha^{L}, \beta^{L}, \mu_{x}\right) d \mu(x), \int j^{\mathrm{vr}}\left(\alpha^{L}, \beta^{L}, \mu_{x}\right) d \mu(x)$, and $\int J\left(\alpha^{L}, \beta^{L}, \mu_{x}\right) d \mu(x)$. In particular, by an argument given in [6]

$$
\begin{aligned}
& \max _{\alpha, \beta \geq 0}[\left.\frac{1}{\alpha}\left(\int j^{\mathrm{fr}}\left(\alpha, \beta, \mu_{x}\right) d \mu(x)-\beta R_{1}\right)\right] \\
&=\int \delta^{\mathrm{fr}}\left(R_{1}, \mu_{x}\right) d \mu(x) \\
& \max _{\alpha, \beta \geq 0}\left[\frac{1}{\alpha}\left(\int j^{\mathrm{vr}}\left(\alpha, \beta, \mu_{x}\right) d \mu(x)-\beta R_{1}\right)\right] \\
&=\inf _{\left\{R_{x}\right\}}\left\{\int \delta^{\mathrm{vr}}\left(R_{x}, \mu_{x}\right) d \mu(x): \int R_{x} d \mu(x) \leq R_{1}\right\} \\
& \max _{\alpha, \beta \geq 0}\left[\frac{1}{\alpha}\left(\int J\left(\alpha, \beta, \mu_{x}\right) d \mu(x)-\beta R_{1}\right)\right] \\
&=\inf _{\left\{R_{x}\right\}}\left\{\int D\left(R_{x}, \mu_{x}\right) d \mu(x): \int R_{x} d \mu(x) \leq R_{1}\right\}
\end{aligned}
$$

when $D\left(R, \mu_{x}\right)$ is a measurable function of $R$ and $x$. Combining the above consequences of the Lagrange duality theorem with Theorems 1-12 gives the following collection of corollaries.

Corollaries 1 and 2 hold for stationary ergodic sources under the conditions of Theorems $1-4$.

$$
\begin{array}{ll}
\text { Corollary 1: } & \delta^{\mathrm{fr}}(R, \mu)=D(R, \mu) . \\
\text { Corollary 2: } & \delta^{\mathrm{vr}}(R, \mu)=D(R, \mu) .
\end{array}
$$

Corollaries 3 and 4 hold for stationary nonergodic sources under the conditions of Theorems 5-12. 
Corollary 3:

$$
\begin{aligned}
\delta^{\mathrm{fr}}(R, \mu) & =\int \delta^{\mathrm{fr}}\left(R, \mu_{x}\right) d \mu(x) \\
& =\int D\left(R, \mu_{x}\right) d \mu(x) \\
& \geq \inf _{\left\{R_{x}\right\}}\left\{\int D\left(R_{x}, \mu_{x}\right) d \mu(x): \int R_{x} d \mu(x) \leq R\right\} \\
& =D(R, \mu) .
\end{aligned}
$$

Corollary 4:

$$
\begin{aligned}
\delta^{\mathrm{vr}} & (R, \mu) \\
& =\inf _{\left\{R_{x}\right\}}\left\{\int \delta^{(\mathrm{vr})}\left(R_{x}, \mu_{x}\right) d \mu(x): \int R_{x} d \mu(x) \leq R\right\} \\
& =\inf _{\left\{R_{x}\right\}}\left\{\int D\left(R_{x}, \mu_{x}\right) d \mu(x): \int R_{x} d \mu(x) \leq R\right\} \\
& =D(R, \mu) .
\end{aligned}
$$

Corollaries 1 and 2 are the fixed- and variable-rate source coding theorems for stationary ergodic sources [5, Theorems 7.2.4, 7.2.5]; Corollary 3 is the fixed-rate source coding theorem for stationary nonergodic sources [22]; and Corollary 4 is the variable-rate source coding theorem for stationary nonergodic sources [23], [6].

As the preceding discussion illustrates, in the case of single-resolution codes, the Lagrange duality theorem results in alternative descriptions, and thus alternative means of understanding our source coding theorems. The Lagrange duality theorem can likewise be applied to cases where $L>1$. As an example, letting $\delta_{\alpha^{L}}\left(R^{L}, \mu\right)$ and $D_{\alpha^{L}}\left(R^{L}, \mu\right)$ be the $L$ dimensional analogies to $\delta(R, \mu)$ and $D(R, \mu)$ yields a variety of additional corollaries, as the following argument illustrates. Let

$$
\begin{aligned}
\delta_{\alpha^{L}}^{\mathrm{fr}}\left(R^{L}, \mu\right) & =\inf _{N} \delta_{\alpha^{L}, N}^{\mathrm{fr}}\left(R^{L}, \mu\right) \\
\delta_{\alpha^{L}}^{\mathrm{vr}}\left(R^{L}, \mu\right) & =\inf _{N} \delta_{\alpha^{L}, N}^{\mathrm{vr}}\left(R^{L}, \mu\right) \\
D_{\alpha^{L}}\left(R^{L}, \mu\right) & =\inf _{N} D_{\alpha^{L}, N}\left(R^{L}, \mu\right),
\end{aligned}
$$

where

$$
\begin{aligned}
& \delta_{\alpha^{L}, N}^{\mathrm{fr}}\left(R^{L}, \mu\right) \\
& =\inf _{Q^{L, N} \in \mathcal{Q}^{\text {fr }}(L, N)}\left\{\frac{1}{N} \alpha^{L} D^{t}\left(\mu, Q^{L, N}\left(X^{N}\right)\right):\right. \\
& \left.\frac{1}{N} R\left(\mu, Q^{L, N}\right) \leq R^{L}\right\} \\
& \delta_{\alpha^{L}, N}^{\mathrm{vr}}\left(R^{L}, \mu\right) \\
& =\inf _{Q^{L, N} \in \mathcal{Q}^{\operatorname{vr}}(L, N)}\left\{\frac{1}{N} \alpha^{L} D^{t}\left(\mu, Q^{L, N}\left(X^{N}\right)\right):\right. \\
& \left.\frac{1}{N} R\left(\mu, Q^{L, N}\right) \leq R^{L}\right\} \\
& D_{\alpha^{L}, N}\left(R^{L}, \mu\right) \\
& =\inf _{q}\left\{\frac{1}{N} E_{\mu^{N} q^{N}} \sum_{\ell=1}^{L} \alpha_{\ell} \rho\left(X^{N}, \boldsymbol{Y}_{\ell}\right):\right. \\
& \left.\frac{1}{N} I_{\mu^{N} q^{N}}\left(X^{N} ; Y_{\ell} \mid Y_{\ell-1}\right) \leq R_{\ell} \forall 1 \leq \ell \leq L\right\} .
\end{aligned}
$$

For example, when $\alpha^{L}=(\alpha, \cdots, \alpha), \delta_{\alpha^{L}, N}^{\mathrm{fr}}\left(R^{L}, \mu\right)$ and $\delta_{\alpha^{L}, N}^{\mathrm{vr}}\left(R^{L}, \mu\right)$ describe the minimal average distortion achievable with a given collection of rate constraints. The parameter vector $\alpha^{L}$ can also be used to describe the proportion of users using each resolution $\ell \in\{1, \cdots, L\}$. In this case, $\delta_{\alpha^{L}}^{\mathrm{fr}}\left(R^{L}, \mu\right)$ and $\delta_{\alpha^{L}}^{\mathrm{vr}}\left(R^{L}, \mu\right)$ describe the minimal expected distortion achievable with rate vector $R^{L}$, where the expectation is taken with respect to the distribution over the resolutions. A simple extension of the above arguments to $\delta_{\alpha^{L}}^{\mathrm{fr}}\left(R^{L}, \mu\right)$ and $\delta_{\alpha^{L}}^{\mathrm{vr}}\left(R^{L}, \mu\right)$ gives the following corollaries.

Corollaries 5 and 6 hold for stationary ergodic sources under the conditions of Theorems 1-4.

Corollary 5: $\delta_{\alpha^{L}}^{\mathrm{fr}}\left(R^{L}, \mu\right)=D_{\alpha^{L}}\left(R^{L}, \mu\right)$.

Corollary 6: $\delta_{\alpha^{L}}^{\mathrm{vr}}\left(R^{L}, \mu\right)=D_{\alpha^{L}}\left(R^{L}, \mu\right)$.

Corollaries 7 and 8 hold for stationary nonergodic sources under the conditions of Theorems 5-12.

Corollary 7

$$
\begin{aligned}
\delta_{\alpha^{L}}^{\mathrm{fr}} & \left(R^{L}, \mu\right) \\
& =\int \delta_{\alpha^{L}}^{\mathrm{fr}}\left(R^{L}, \mu_{x}\right) d \mu(x) \\
& =\int D_{\alpha^{L}}\left(R^{L}, \mu_{x}\right) d \mu(x) \\
& \geq \inf _{\left\{R_{x}^{L}\right\}}\left\{\int D_{\alpha^{L}}\left(R_{x}^{L}, \mu_{x}\right) d \mu(x): \int R_{x}^{L} d \mu(x) \leq R^{L}\right\} \\
& =D_{\alpha^{L}}\left(R^{L}, \mu\right) .
\end{aligned}
$$

\section{Corollary 8:}

$$
\begin{aligned}
& \delta_{\alpha^{L}}^{\mathrm{vr}}\left(R^{L}, \mu\right) \\
& =\inf _{\left\{R_{x}^{L}\right\}}\left\{\int \delta_{\alpha^{L}}^{(\mathrm{vr})}\left(R_{x}^{L}, \mu_{x}\right) d \mu(x): \int R_{x}^{L} d \mu(x) \leq R^{L}\right\} \\
& =\inf _{\left\{R_{x}^{L}\right\}}\left\{\int D_{\alpha^{L}}\left(R_{x}^{L}, \mu_{x}\right) d \mu(x): \int R_{x}^{L} d \mu(x) \leq R^{L}\right\} \\
& =D_{\alpha^{L}}\left(R^{L}, \mu\right) .
\end{aligned}
$$

The above results demonstrate one interpretation of the weighted distortion-rate and operational weighted distortionrate functions as Lagrangians for minimizing the weighted sum of distortions subject to a collection of constraints on the rates. The same functions could likewise be interpreted as Lagrangians for the minimization of the weighted sum of rates subject to a collection of constraints on the distortions, a minimization of the weighted sum of distortionrate Lagrangians (at the same or-more likely-differing slopes), or in fact a minimization of any combination of rates, distortions, or Lagrangians subject to constraints on the remaining quantities. Thus for example, the weighted distortion-rate function can be used to find: the minimal rate $R_{2}$ needed to achieve a particular distortion $D_{2}<D_{1}$ given $\left(R_{1}, D_{1}\right)=\left(R\left(D_{1}\right), D_{1}\right)$; the minimal rate $R_{1}$ needed to achieve a particular distortion $D_{1}>D_{2}$ given that $\left(R_{2}, D_{2}\right)=$ $\left(R\left(D_{2}\right), D_{2}\right)$; the minimal $D_{1}+\lambda R_{1}$ given that $D_{2}=D\left(R_{2}\right)$; and so on; thereby encompassing a variety of multiresolution source coding problems considered by previous authors into 
a single formulation. This variety of interpretations illustrates one of the benefits of the Lagrangian approach.

Finally, Theorems 1-12 lead to the following characterization of the set of achievable rate distortion vectors $\mathcal{R}^{\mathrm{fr}, L}(\mu)$ and $\mathcal{R}^{\mathrm{vr}, L}(\mu)$.

Corollary 9: If $[A, \mu]$ is stationary and ergodic, then under the conditions of Theorems $1-4$ the rate distortion vector $\left(r^{L}, d^{L}\right)$ is achievable by both fixed- and variable-rate codes if there exists a test channel $q$ such that

$I_{\mu q}\left(\mathcal{X} ; \mathcal{Y}^{\ell}\right) \leq \sum_{i=1}^{\ell} r_{i} \quad$ and $\quad \lim _{N \rightarrow \infty} \frac{1}{N} E_{\mu^{N} q^{N}} \rho\left(X^{N} ; \boldsymbol{Y}_{\ell}\right) \leq d_{\ell}$

for all $1 \leq \ell \leq L$.

Proof: By Lemma 3, it is sufficient to show that any rate distortion vector $\left(r^{L}, d^{L}\right)$ such that $r_{\ell}=I_{\mu q}\left(\mathcal{X} ; \mathcal{Y}_{\ell} \mid \mathcal{Y}^{\ell-1}\right)$ and $d_{\ell}=\lim _{N \rightarrow \infty}(1 / N) E_{\mu^{N} q^{N}} \rho\left(X^{N}, Y_{\ell}\right)$ for some fixed test channel $q$ is achievable by both fixed- and variable-rate coding. This result is an immediate consequence of Theorems 2 and 3 and Lemma 6 since

$$
\begin{aligned}
\sum_{\ell=1}^{L}\left(\alpha_{\ell} d_{\ell}+\beta_{\ell} r_{\ell}\right)= & \lim _{N \rightarrow \infty} \frac{1}{N} \sum_{\ell=1}^{L}\left(\alpha_{\ell} E_{\mu^{N} q^{N}} \rho\left(X^{N}, \boldsymbol{Y}_{\ell}\right)\right. \\
& \left.+\beta_{\ell} I_{\mu^{N} q^{N}}\left(X^{N} ; \boldsymbol{Y}_{\ell} \mid \boldsymbol{Y}^{\ell-1}\right)\right) \\
\geq & \lim _{N \rightarrow \infty} J_{N}\left(\alpha^{L}, \beta^{L}, \mu\right) \\
= & J\left(\alpha^{L}, \beta^{L}, \mu\right)
\end{aligned}
$$

for all $\alpha^{L}, \beta^{L} \geq \mathbf{0}$.

Corollary 10: If $[A, \mu]$ is stationary and nonergodic, then under the conditions of Theorems 5-12, the rate distortion vector $\left(r^{L}, d^{L}\right)$ is achievable by a fixed-rate code if there exists a test channel $q$ such that

$$
I_{\mu_{x} q}\left(\mathcal{X} ; \mathcal{Y}^{\ell}\right) \leq \sum_{i=1}^{\ell} r_{i} \quad \mu \text {-a.e. }
$$

and

$$
\int \lim _{N \rightarrow \infty} \frac{1}{N} E_{\mu_{x}^{N} q^{N}} \rho\left(X^{N}, \boldsymbol{Y}_{\ell}\right) d \mu(x) \leq d_{\ell}
$$

for all $1 \leq \ell \leq L$.

Proof: As with Corollary 9, it is sufficient to prove that $r_{\ell} \geq I_{\mu_{x} q}\left(\mathcal{X} ; \mathcal{Y}_{\ell} \mid \mathcal{Y}^{\ell-1}\right) \mu$-almost everywhere and

$$
d_{\ell} \geq \int \lim _{N \rightarrow \infty} \frac{1}{N} E_{\mu_{x}^{N} q^{N}} \rho\left(X^{N} \boldsymbol{Y}_{\ell}\right) d \mu(x)
$$

for all $1 \leq \ell \leq L$ imply that $\left(r^{L}, d^{L}\right)$ is achievable by a fixed-rate code. Under these conditions

$$
\begin{aligned}
& \sum_{\ell=1}^{L}\left(\alpha_{\ell} d_{\ell}+\beta_{\ell} r_{\ell}\right) \\
& \geq \sum_{\ell=1}^{L}\left(\alpha_{\ell} \int \lim _{N \rightarrow \infty} \frac{1}{N} E_{\mu_{x}^{N} q^{N} \rho\left(X^{N}, \boldsymbol{Y}_{\ell}\right) d \mu(x)}\right. \\
& \left.\quad+\beta_{\ell} \int I_{\mu_{x} q}\left(\mathcal{X} ; \mathcal{Y}_{\ell} \mid \mathcal{Y}^{\ell-1}\right) d \mu(x)\right)
\end{aligned}
$$

$$
\begin{aligned}
& =\int \lim _{N \rightarrow \infty} \frac{1}{N} \sum_{\ell=1}^{L}\left(\alpha_{\ell} E_{\mu_{x}^{N} q^{N}} \rho\left(X^{N}, \boldsymbol{Y}_{\ell}\right)\right. \\
& \left.+\beta_{\ell} I_{\mu_{x}^{N} q^{N}}\left(X^{N} ; \boldsymbol{Y}_{\ell} \mid \boldsymbol{Y}^{\ell-1}\right)\right) d \mu(x) \\
& \geq \int \lim _{N \rightarrow \infty} \inf _{q} \frac{1}{N}\left\{\sum _ { \ell = 1 } ^ { L } \left(\alpha_{\ell} E_{\mu_{x}^{N} q^{N}} \rho\left(X^{N}, \boldsymbol{Y}_{\ell}\right)\right.\right. \\
& \left.+\beta_{\ell} I_{\mu_{x}^{N} q^{N}}\left(X^{N} ; \boldsymbol{Y}_{\ell} \mid \boldsymbol{Y}^{\ell-1}\right)\right): \\
& \left.\frac{1}{N} I_{\mu_{x}^{N} q^{N}}\left(X^{N} ; \boldsymbol{Y}_{\ell} \mid \boldsymbol{Y}^{\ell-1}\right) \leq r_{\ell} \forall 1 \leq \ell \leq L\right\} d \mu(x) \\
& =\int \lim _{N \rightarrow \infty} J_{N}^{r^{L}}\left(\alpha^{L}, \beta^{L}, \mu_{x}\right) d \mu(x) \\
& =\int J^{r^{L}}\left(\alpha^{L}, \beta^{L}, \mu_{x}\right) d \mu(x) \\
& =j^{\mathrm{fr}, r^{L}}\left(\alpha^{L}, \beta^{L}, \mu\right) \\
& \geq j^{\mathrm{fr}}\left(\alpha^{L}, \beta^{L}, \mu\right)
\end{aligned}
$$

for any $\alpha^{L}, \beta^{L} \geq \mathbf{0}$. The last three steps are given by Lemma 6, Theorem 9, and (11).

Corollary 11: If $[A, \mu]$ is stationary and nonergodic, then under the conditions of Theorems 5-12, the rate-distortion vector $\left(r^{L}, d^{L}\right)$ is achievable by a variable-rate code if there exists a test channel $q$ such that

$$
\int I_{\mu_{x} q}\left(\mathcal{X} ; \mathcal{Y}^{\ell}\right) d \mu(x) \leq \sum_{\ell=1}^{L} r_{\ell}
$$

and

$$
\int \lim _{N \rightarrow \infty} \frac{1}{N} E_{\mu_{x}^{N} q^{N}} \rho\left(X^{N}, \boldsymbol{Y}_{\ell}\right) d \mu(x) \leq d_{\ell}
$$

for all $1 \leq \ell \leq L$

Proof: By Lemma 3, it is sufficient to show that

$$
r_{\ell} \geq \int I_{\mu_{x} q}\left(\mathcal{X} ; \mathcal{Y}_{\ell} \mid \mathcal{Y}^{\ell-1}\right) d \mu(x)
$$

and

$$
d_{\ell} \geq \int \lim _{N \rightarrow \infty} \frac{1}{N} E_{\mu_{x}^{N} q^{N}} \rho\left(X^{N}, Y_{\ell}\right) d \mu(x)
$$

for all $1 \leq \ell \leq L$ implies $\left(r^{L}, d^{L}\right) \in \mathcal{R}^{\mathrm{vr}, L}(\mu)$. For any such rate distortion vector

$$
\begin{aligned}
\sum_{\ell=1}^{L}\left(\alpha_{\ell} d_{\ell}+\beta_{\ell} r_{\ell}\right) \geq & \int \lim _{N \rightarrow \infty} \frac{1}{N} \sum_{\ell=1}^{L}\left(\alpha_{\ell} E_{\mu_{x}^{N} q^{N}} \rho\left(X^{N}, \boldsymbol{Y}_{\ell}\right)\right. \\
& \left.+\beta_{\ell} I_{\mu_{x}^{N}} q^{N}\left(X^{N} ; \boldsymbol{Y}_{\ell} \mid \boldsymbol{Y}^{\ell-1}\right)\right) d \mu(x) \\
\geq & \int \lim _{N \rightarrow \infty} J_{N}\left(\alpha^{L}, \beta^{L}, \mu_{x}\right) d \mu(x) \\
= & \int J\left(\alpha^{L}, \beta^{L}, \mu_{x}\right) d \mu(x) \\
= & J\left(\alpha^{L}, \beta^{L}, \mu\right) \\
= & j^{\operatorname{vr}}\left(\alpha^{L}, \beta^{L}, \mu\right)
\end{aligned}
$$

for any $\alpha^{L}, \beta^{L} \geq \mathbf{0}$, where the last three steps are given by Lemma 6, Theorem 8, and Theorem 11, respectively. 
For a discrete memoryless source, Corollary 9 is the forward half of Rimoldi's source coding theorem [16], here generalized from discrete memoryless sources to stationary ergodic sources on Polish alphabets, of which discrete memoryless sources are a special case. Corollaries 10 and 11 further generalize this result for fixed- and variable-rate coding on stationary ergodic and stationary nonergodic sources again on Polish alphabets.

\section{ANALYSIS}

As discussed in the previous section, the given distortionrate results are natural extensions of single-resolution sourcecoding results for stationary ergodic and stationary nonergodic sources on Polish alphabets. As a result, many existing techniques for calculating and approximating single-resolution distortion-rate curves likewise generalize to the case of multiresolution source coding. An outline of the analysis of $J\left(\alpha^{L}, \beta^{L}, \mu\right)$ follows.

Finding the achievable distortion-rate region for a given source involves a search for the conditional distribution $q$ that gives the weighted distortion-rate function $J\left(\alpha^{L}, \beta^{L}, \mu\right)$. For simplicity, consider a memoryless source. Then by (15) and (16),

$J\left(\alpha^{L}, \beta^{L}, \mu\right)=\inf _{q} \sum_{\ell=1}^{L}\left(\alpha_{\ell} E_{\mu q} \rho\left(X, Y_{\ell}\right)+\beta_{\ell} I_{\mu q}\left(X ; Y_{\ell} \mid Y^{\ell-1}\right)\right)$

where the infimum is taken over all conditional distributions $q$ with the desired input and output alphabets. The above equation represents a constrained minimization, where the constraints imposed are precisely those constraints necessary for $q$ to be a legitimate conditional distribution. Using Lagrangians, the above constrained minimization may be replaced with an unconstrained minimization and differentiated, yielding a parametric description of the conditional distribution $q$. For $L=2$ and a finite alphabet, the above technique yields the following collection of equations:

$$
\begin{aligned}
q\left(y_{1} \mid x\right) & =\lambda(x) q\left(y_{1}\right) \omega\left(x, y_{1}\right)^{-\frac{\beta_{2}}{\beta_{1}}} e^{\frac{\alpha_{1}}{\beta_{1}} \rho\left(x, y_{1}\right)} \\
q\left(y_{2} \mid y_{1}, x\right) & =\omega\left(x, y_{1}\right) q\left(y_{2} \mid y_{1}\right) e^{\frac{\alpha_{2}}{\beta_{2}} \rho\left(x, y_{2}\right)} \\
\lambda(x) & =\left[\sum_{y_{1}} q\left(y_{1}\right) \omega\left(x, y_{1}\right)^{-\frac{\beta_{2}}{\beta_{1}}} e^{\frac{\alpha_{1}}{\beta_{1}} \rho\left(x, y_{1}\right)}\right]^{-1} \\
\omega\left(x, y_{1}\right) & =\left[\sum_{y_{2}} q\left(y_{2} \mid y_{1}\right) e^{\frac{\alpha_{2}}{\beta_{2}} \rho\left(x, y_{2}\right)}\right]^{-1}
\end{aligned}
$$$$
\sum_{x} \mu(x) \lambda(x) \omega\left(x, y_{1}\right)^{-\frac{\beta_{2}}{\beta_{1}}} e^{\frac{\alpha_{1}}{\beta_{1}} \rho\left(x, y_{1}\right)} \leq 1
$$$$
\text { with equality iff } q\left(y_{1}\right)>0
$$$$
\sum_{x} \mu(x) \omega\left(x, y_{1}\right) q\left(y_{1} \mid x\right) e^{\frac{\alpha_{2}}{\beta_{2}} \rho\left(x, y_{2}\right)} \leq q\left(y_{1}\right)
$$$$
\text { with equality iff } q\left(y_{2} \mid y_{1}\right)>0 \text {. }
$$

Here $q\left(y_{1}\right)$ and $q\left(y_{2} \mid y_{1}\right)$ represent the marginal on $y_{1}$ and conditional distribution on $y_{2}$ given $y_{1}$, respectively, and are thus defined as

$$
\begin{gathered}
q\left(y_{1}\right)=\sum_{x} \mu(x) q\left(y_{1} \mid x\right) \\
q\left(y_{2} \mid y_{1}\right)=\frac{\sum_{x} \mu(x) q\left(y_{1} \mid x\right) q\left(y_{2} \mid y_{1}, x\right)}{q\left(y_{1}\right)} .
\end{gathered}
$$

The results for continuous alphabet sources are similar, with integrals replacing the above summations. Equations for arbitrary $L$ can likewise be obtained. For finite alphabet sources, (21)-(26) can be rearranged into a collection of (mostly) linear equations and solved.

The following discussion gives the distortion-rate region for two discrete-alphabet examples. Both of the examples considered rely upon a source that has its origin in [24] and appears in [13] and [14] as the first example of a source that can be proven not to be successively refinable. Example 1 treats the two-resolution distortion-rate region for this source while Example 2 treats the two-resolution distortion-rate region for a stationary nonergodic source with two ergodic modes, one of which is the source treated in the first example.

Example 1. A Stationary Ergodic Source: Let $\mathcal{X}=\hat{\mathcal{X}}=$ $\{1,2,3\}$ and use $\rho(x, y)=|x-y|$. Now consider i.i.d. samples of a source with probability mass function $\mu=$ $\{(1-p) / 2, p,(1-p) / 2\}$ for any $p<3-2 \sqrt{2}$. The example given here uses $p=0.171$. The solution to the above collection of equations for a source drawn from this distribution is tedious but not conceptually difficult. The procedure parallels the strategy in [5] for single-resolution codes. A collection of graphs showing the resulting distortion-rate performance, both in its entirety and blown up for closer observation, appears in Fig. 3. Each graph in Fig. 3 shows five curves. The lowest curve is the single-resolution distortion-rate curve for the given source. Each higher curve shows the optimal secondresolution performance $\left(R_{1}+R_{2}, D_{2}\right)$ for a family of codes sharing a fixed first-resolution coding performance $\left(R_{1}, D_{1}\right)$ such that $D_{1}=D\left(R_{1}\right)$. That is, the first-resolution coding performance achieves the single-resolution distortion-rate bound. The four curves correspond to four different values of the first-resolution coding rate $R_{1}$. The four curves correspond to $R_{1} \in\{0.098,0.193,0.319,0.343\}$. As demonstrated in Fig. 3, the difference between the optimal single-resolution coding performance and the optimal multiresolution coding performance for this source-which stood for many years as the only documented example of a source that is not successively refinable-is extremely small. In fact, in two of the curves given in Fig. 3 (corresponding to $R_{1} \in\{0.319,0.343\}$ ), there is no penalty in second-resolution performance associated with constraining the first-resolution performance to achieve the single-resolution distortion-rate bound. Fig. 4 shows, for each distortion $D_{2}$, the increase in rate suffered in the second resolution given the constraint imposed on the first-resolution coding performance. The maximal difference varies as a function of the first-resolution rate and distortion but nowhere exceeds 0.0026 bits per symbol in this example. This tiny gap serves to remind us that the fact that a source is not successively refinable proves only that there is a penalty to be paid for 

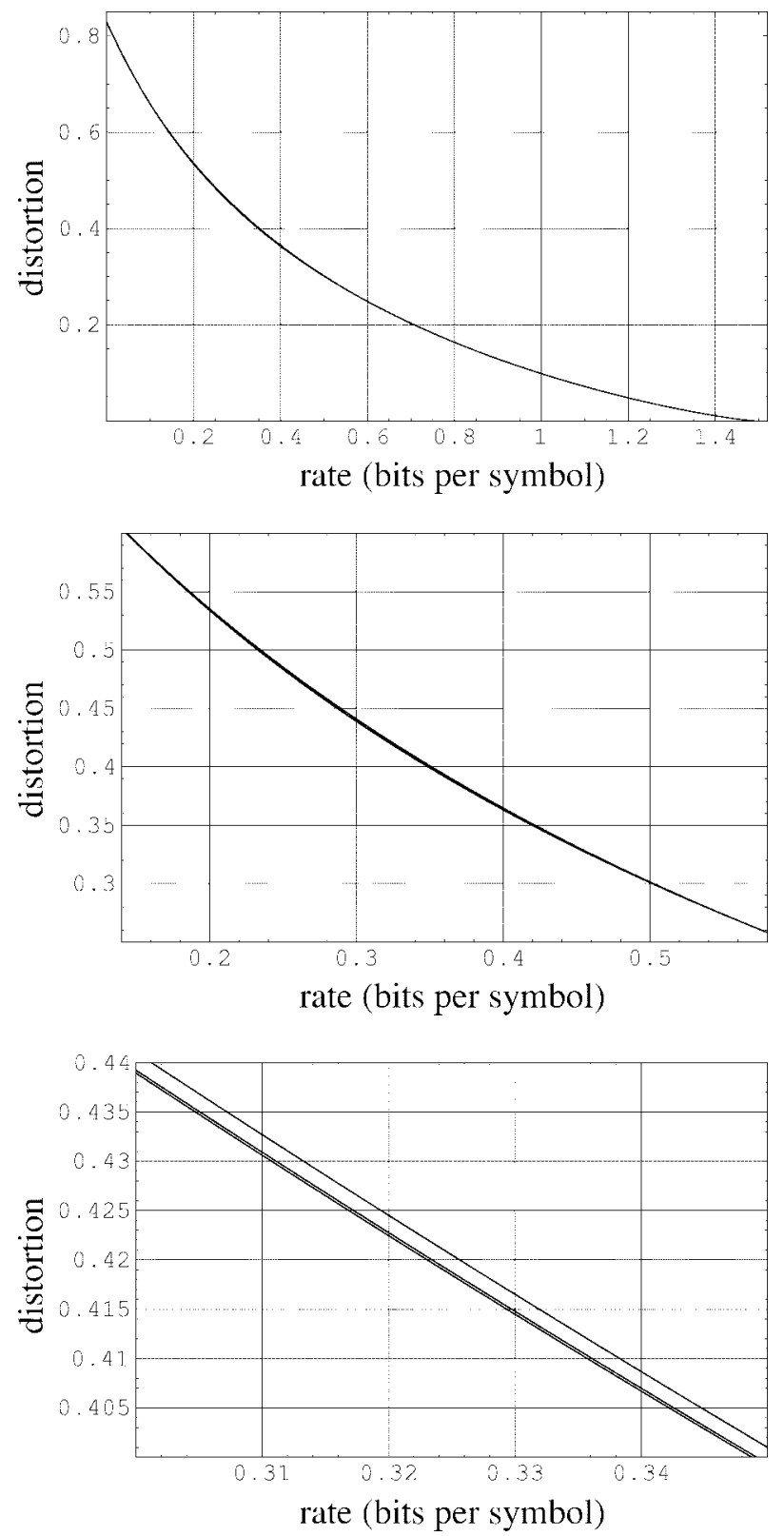

Fig. 3. Distortion-rate results for i.i.d. samples from a source with input and output alphabets $\mathcal{X}=\hat{\mathcal{X}}=\{1,2,3\}$ and probability mass function $\{(1-p) / 2, p,(1-p) / 2\}, p=0.171$. Each graph shows five curves. The lowest curve is the single-resolution distortion-rate curve $D(R)$ for the given source. Each higher curve describes the optimal second-resolution performance $\left(R_{1}+R_{2}, D_{2}\right)$ for codes with first-resolution coding performance equal to $\left(R_{1}, D\left(R_{1}\right)\right)$-in particular, the four curves correspond to $R_{1} \in\{0.098,0.193,0.319,0.343\}$. Two of the curves $\left(R_{1} \in\{0.319,0.343\}\right)$, give second-resolution performance everywhere identical to the single-resolution distortion-rate bound $\left(D_{2}=D\left(R_{1}+R_{2}\right)\right)$. The performance degradation in the other two cases is so small as to be almost unobservable at all but the highest magnification $(20 \times)$.

describing the source in an embedded manner but in no way implies that this penalty is high. The reminder is reassuring in light of [15], which proves a symmetric, bimodal Gaussian mixture not to be successively refinable, thereby suggesting that most real sources are likely not successively refinable.

The results in Example 1 agree with those of [13] and [14], which describe the conditions under which the given source is and is not successively refinable. This work goes further

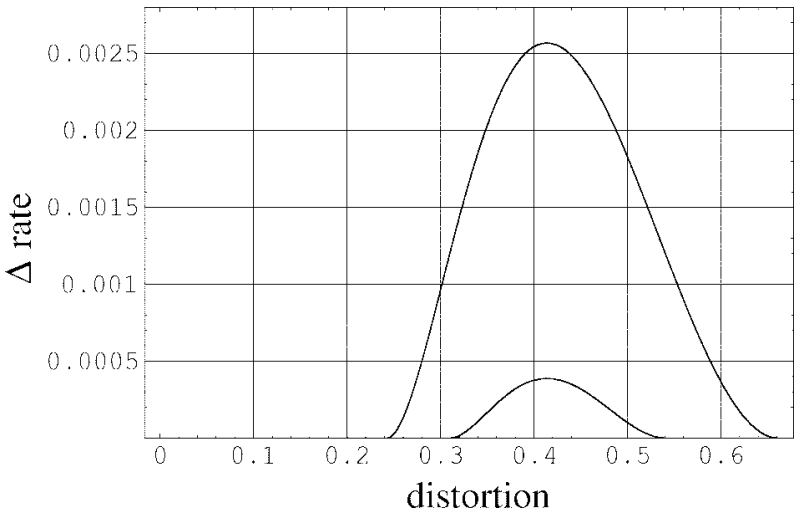

Fig. 4. Distortion-rate results for i.i.d. samples from a source with input and output alphabets $\mathcal{X}=\hat{\mathcal{X}}=\{1,2,3\}$ and probability mass function $\mu=\{(1-p) / 2, p,(1-p) / 2\}$. The given curves use $p=0.171$. The curves show, for each distortion value, the difference in optimal rate between the performance bound on a single-resolution code and the performance bound on a two-resolution code with a fixed first-resolution performance sitting on the distortion-rate bound.

than [13] and [14] to find the distortion-rate region when successive refinement fails. Notice that while the solution to the distortion-rate equations for this particular source is new, the source is discrete and memoryless and therefore falls into the class of sources considered by Rimoldi [16].

In contrast to Example 1, Example 2 treats a stationary nonergodic source, and therefore relies upon the new distortion-rate theorems given in this work.

Example 2. A Stationary Nonergodic Source: Let $\mathcal{X}=$ $\hat{\mathcal{X}}=\{1,2,3\}$ and $\rho(x, y)=|x-y|$ as in the previous example. Suppose that the samples $X_{1}, X_{2}, \cdots$ are drawn i.i.d. according to one of two possible distributions, $\mu_{1}(x)$ or $\mu_{2}(x)$, where a single distribution $\mu_{i}(x), i \in\{1,2\}$ is chosen at the beginning of time and used for all samples, but the choice of $i$ is itself random. The example that follows treats the case where

$$
\begin{aligned}
& \mu_{1}(x)=\{(1-p) / 2, p,(1-p) / 2\}, \\
& \mu_{2}(x)=\{1-p, p, 0\}
\end{aligned}
$$

$p=0.171$, and the two distributions appear with equal probability.

The solution for this example begins with the observation that the two-resolution distortion-rate regions for both $\mu_{1}(x)$ and $\mu_{2}(x)$ are known. In particular, the solution for the two-resolution distortion-rate region for $\mu_{1}(x)$ is effectively given in Example 1. Further, the two-resolution distortionrate region for $\mu_{2}(x)$ with distortion measure $\rho(x, y)=$ $|x-y|$ is equivalent to the two-resolution distortion-rate region for a Bernoulli $(p)$ source with Hamming distortion measure. The two-resolution distortion-rate region for the $\operatorname{Bernoulli}(p)$ source with Hamming distortion measure is given in [13] and [14], where that source is proven to be successively refinable.

The remainder of the solution to the two-resolution distortion-rate region for the given stationary nonergodic source derives from Theorem 8 , which effectively states that the weighted distortion-rate function of a stationary nonergodic source equals the expected value of the weighted distortionrate functions for the source's ergodic modes, where the 

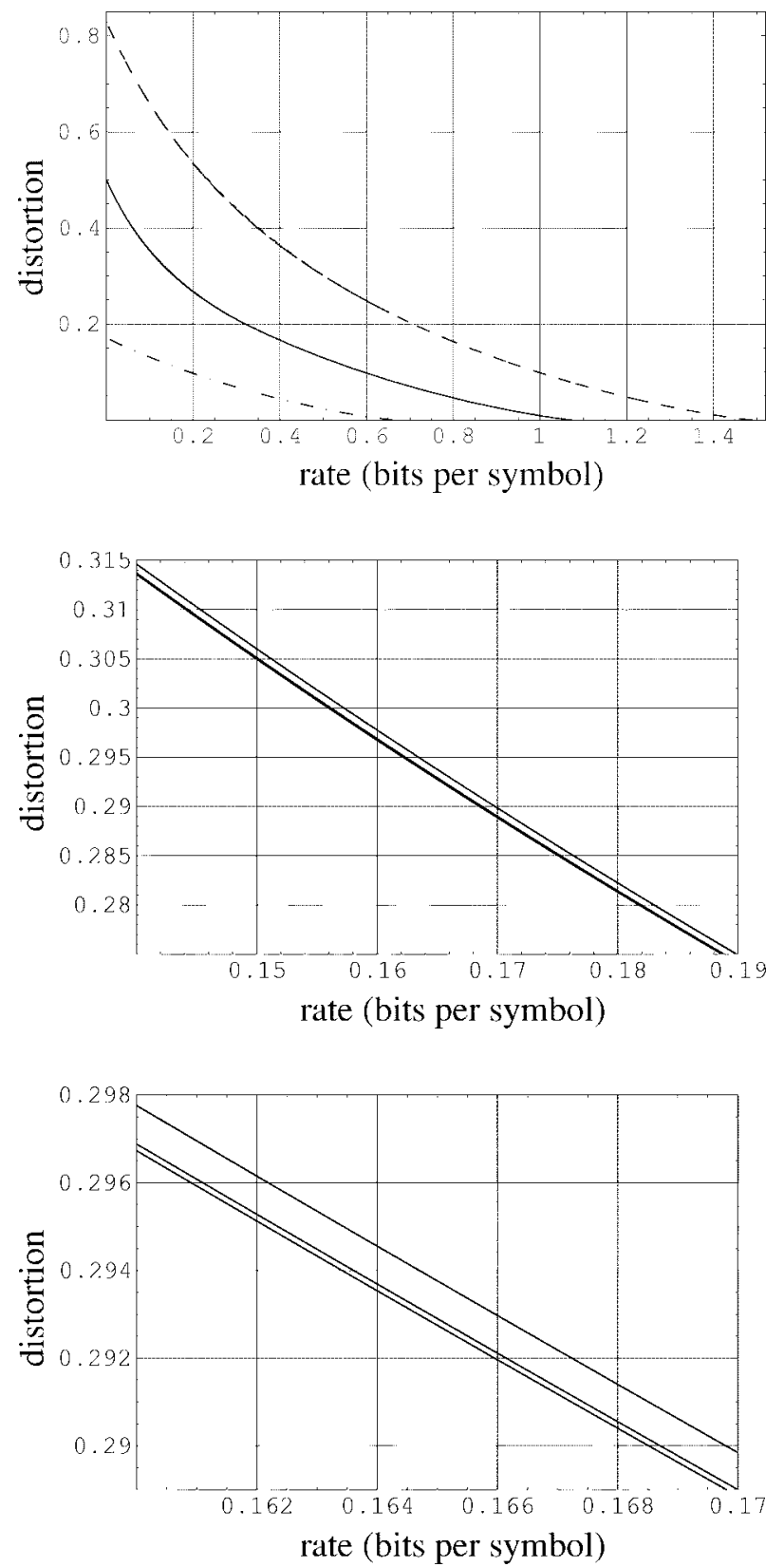

Fig. 5. Distortion-rate results for a nonergodic source with input and output alphabets $\mathcal{X}=\hat{\mathcal{X}}=\{1,2,3\}$. The source has two ergodic modes, $\mu_{1}(x)$ and $\mu_{2}(x)$, each of probability $1 / 2$. The ergodic modes are $\mu_{1}=\{(1-p) / 2, p,(1-p) / 2\}$ and $\mu_{2}=\{1-p, p, 0\}$. The given curves use $p=0.171$. The top graph shows two-resolution results for $\mu_{1}(x)$ (top set of curves), $\mu_{2}(x)$ (bottom set of curves), and the nonergodic source achieved by the nonergodic mixture of $\mu_{1}(x)$ and $\mu_{2}(x)$ (middle set of curves). The remaining two graphs focus on the nonergodic source. The results for each source include five curves. The lowest curve is the single-resolution distortion-rate curve for the given source. The higher curves describe the optimal second-resolution performance for all codes with a particular fixed first-resolution coding performance achieving the single-resolution distortion-rate bound.

expectation is taken over points of equal "slope" or direction $\left(\alpha^{2}, \beta^{2}\right)$. Thus in this example,

$$
J\left(\alpha^{2}, \beta^{2}, \mu\right)=\left(J\left(\alpha^{2}, \beta^{2}, \mu_{1}\right)+J\left(\alpha^{2}, \beta^{2}, \mu_{2}\right)\right) / 2 .
$$

The two-resolution distortion-rate results for this source appear in Fig. 5. The resulting nonergodic source is not successively refinable, as could have been predicted since the nonergodic source includes an ergodic mode of nonzero measure that is not successively refinable.

Example 2 lends some interesting insights. In particular, the following properties arise under the conditions of Theorems 5-12.

- The weighted distortion-rate function of a stationary nonergodic source is a simple combination of the weighted distortion-rate functions of the source's ergodic modes.

- Source $[A, \mu]$ is successively refinable if and only if $\left[A, \mu_{x}\right]$ is successively refinable almost-surely.

- The maximum "penalty" associated with multiresolution coding on a stationary nonergodic source is bounded by the maximum penalty over all of the source's ergodic modes. For example, the increase in rate associated with achieving distortion $D_{2}$ in the second resolution of a tworesolution code that achieves first-resolution performance $\left(R_{1}, D\left(R_{1}\right)\right.$ ) (see Fig. 4) is no greater than the greatest corresponding increase in rate experienced by the source's ergodic modes.

\section{Distortion-RATE Proofs}

This section begins with a proof of a general converse for the theorems described in Section III. In particular, the converse demonstrates that for any stationary source there does not exist a fixed- or variable-rate code with performance surpassing the weighted distortion-rate function, and, similarly, that there does not exist a fixed-rate- $R^{L}$ code with performance surpassing the rate- $R^{L}$ weighted distortion-rate function. The remainder of the section is dedicated to proving a variety of achievability results. Each achievability proof is given in a sequence of lemmas, many of which are used in more than one proof. The proofs appear in three subsections. The first subsection treats general properties of $j^{\mathrm{fr}}\left(\alpha^{L}, \beta^{L}, \mu\right)$, $j^{\mathrm{fr}, R^{L}}\left(\alpha^{L}, \beta^{L}, \mu\right)$, and $j^{\mathrm{vr}}\left(\alpha^{L}, \beta^{L}, \mu\right)$. The second and third sections treat stationary ergodic and stationary nonergodic sources, respectively.

Lemma 7 (Converse): Let $[A, \mu]$ be a stationary source with weighted distortion-rate functions $J^{R^{L}}\left(\alpha^{L}, \beta^{L}, \mu\right)$ and $J\left(\alpha^{L}, \beta^{L}, \mu\right)$. Then for any $N \geq 1$ there does not exist a quantizer $Q^{L, N} \in \mathcal{Q}^{\mathrm{fr}, R^{L}}(L, N)$ such that

$$
(1 / N)\left[\alpha^{L} D^{t}\left(\mu, Q^{L, N}\right)+\beta^{L}\left(N R^{L}\right)^{t}\right]<J^{R^{L}}\left(\alpha^{L}, \beta^{L}, \mu\right) .
$$

Similarly, for any $N \geq 1$ there does not exist a quantizer $Q^{L, N} \in \mathcal{Q}^{\mathrm{vr}}(L, N) \supseteq \mathcal{Q}^{\mathrm{fr}}(L, N)$ such that

$$
(1 / N)\left[\alpha^{L} \boldsymbol{D}^{t}\left(\mu, Q^{L, N}\right)+\beta^{L} \boldsymbol{R}^{t}\left(\mu, Q^{L, N}\right)\right]<J\left(\alpha^{L}, \beta^{L}, \mu\right) .
$$

Thus

and

$$
j^{\mathrm{fr}, R^{L}}\left(\alpha^{L}, \beta^{L}, \mu\right) \geq J^{R^{L}}\left(\alpha^{L}, \beta^{L}, \mu\right)
$$

$$
j^{\mathrm{fr}}\left(\alpha^{L}, \beta^{L}, \mu\right) \geq j^{\mathrm{vr}}\left(\alpha^{L}, \beta^{L}, \mu\right) \geq J\left(\alpha^{L}, \beta^{L}, \mu\right) .
$$


Proof: Consider the second result first. Suppose that there exist an integer $N \geq 1$ and a quantizer $Q^{L, N} \in$ $\mathcal{Q}^{\mathrm{vr}}(L, N)$ such that

$$
(1 / N)\left[\alpha^{L} \boldsymbol{D}^{t}\left(\mu, Q^{L, N}\right)+\beta^{L} \boldsymbol{R}^{t}\left(\mu, Q^{L, N}\right)\right]<J\left(\alpha^{L}, \beta^{L}, \mu\right)-\epsilon
$$

for some $\epsilon>0$. Let $q^{N}$ be the $N$-dimensional degenerate test channel that deterministically maps each $x^{N} \in A^{N}$ to the vector $\boldsymbol{y}^{L} \in\left(\hat{A}^{N}\right)^{L}$ such that $Q^{L, N}\left(x^{N}\right)=y^{L}$, and let $\nu^{N}$ be the marginal on $\boldsymbol{Y}^{L}$ associated with joint distribution $\mu^{N} q^{N}$. Then, by Shannon's lossless source coding theorem,

$$
E_{\mu}\left|Q_{(\ell)}^{N}\left(X^{N}\right)\right| \geq H_{\nu^{N}}\left(\boldsymbol{Y}_{\ell} \mid \boldsymbol{Y}^{\ell-1}\right) \geq I_{\mu^{N} q^{N}}\left(X^{N} ; \boldsymbol{Y}_{\ell} \mid \boldsymbol{Y}^{\ell-1}\right)
$$

for each $1 \leq \ell \leq L$. Further

$$
E_{\mu^{N} q^{N}} \rho\left(X^{N}, Y_{\ell}\right)=\boldsymbol{D}\left(\mu, Q^{L, N}\right)
$$

for all $1 \leq \ell \leq L$. Thus

$$
\begin{aligned}
J_{N}\left(\alpha^{L}, \beta^{L}, \mu\right) \leq & \frac{1}{N} \sum_{\ell=1}^{L}\left(\alpha_{\ell} E_{\boldsymbol{\mu}^{N} q^{N}} \rho\left(X^{N}, \boldsymbol{Y}_{\ell}\right)\right. \\
& \left.+\beta_{\ell} I_{\mu^{N} q^{N}}\left(X^{N} ; \boldsymbol{Y}_{\ell} \mid \boldsymbol{Y}^{\ell-1}\right)\right) \\
\leq & \frac{1}{N}\left(\alpha^{L} \boldsymbol{D}^{t}\left(\mu, Q^{L, N}\right)+\beta^{L} \boldsymbol{R}^{t}\left(\mu, Q^{L, N}\right)\right) \\
< & J\left(\alpha^{L}, \beta^{L}, \mu\right)-\epsilon
\end{aligned}
$$

which contradicts with the definition of $J\left(\alpha^{L}, \beta^{L}, \mu\right)$ found in (15).

The proof of the first result is almost identical. In this case, the test channel $q^{N}$ is matched to a quantizer $Q^{L, N} \in$ $\mathcal{Q}^{\mathrm{fr}, R^{L}}(L, N)$, which implies that

$$
R_{\ell}>(1 / N)\left|Q_{(\ell)}^{N}\left(x^{N}\right)\right| \geq(1 / N) I_{\mu^{N} q^{N}}\left(X^{N} ; \boldsymbol{Y}_{\ell} \mid \boldsymbol{Y}^{\ell-1}\right)
$$

for each $\ell \in\{1, \cdots, L\}$. The remainder of the proof follows.

\section{A. General Properties}

Lemma 8 demonstrates that the infima in the definitions of the $N$ th-order weighted fixed-rate, fixed-rate- $R^{L}$, and variable-rate operational distortion-rate functions can be restricted to quantizers with encoders using weighted nearest neighbor decision rules. The following definitions are useful for describing that result. Let $\mathcal{K}^{\mathrm{fr}}(L, N)$ be the set of finite, $L$-resolution, fixed-rate codebooks so that for any

$$
\mathcal{C}^{L, N}=\left\{\mathcal{C}_{(1)}^{N}, \cdots, \mathcal{C}_{(L)}^{N}\right\} \in \mathcal{K}^{\mathrm{fr}}(L, N)
$$

$\mathcal{C}^{L, N}$ contains the $L$ codebooks of $Q^{L, N}$ for some $Q^{L, N} \in$ $\mathcal{Q}^{\mathrm{fr}}(L, N)$. Similarly, let $\mathcal{K}^{\mathrm{fr}, R^{L}}(L, N)$ be the set of finite, $L$-resolution, fixed-rate- $R^{L}$ codebooks corresponding to quantizers in $\mathcal{Q}^{\mathrm{fr}, R^{L}}(L, N)$ and let $\mathcal{K}^{\mathrm{vr}}(L, N)$ be the set of finite or countable, $L$-resolution, variable-rate codebooks corresponding to quantizers in $\mathcal{Q}^{\mathrm{vr}}(L, N)$. Further, for any codebook $\mathcal{C}^{L, N}$, let $\boldsymbol{y}^{L} \in \mathcal{C}^{L, N}$ be used to designate that $\boldsymbol{y}_{1} \in \mathcal{C}_{(1)}^{N}$ and $\boldsymbol{y}_{\ell} \in \mathcal{C}_{(\ell)}^{N}\left(\boldsymbol{y}_{\ell-1}\right)$ for all $2 \leq \ell \leq L$.
Lemma 8 (Nearest Neighbor Encoding): For any $L$ resolution codebook $\mathcal{C}^{L, N}$ such that $\mathcal{C}^{L, N} \in \mathcal{K}^{\mathrm{fr}}(L, N)$ or $\mathcal{C}^{L, N} \in \mathcal{K}^{\mathrm{vr}}(L, N)$, the infimum

$d_{\left(\alpha^{L}, \beta^{L}\right)}\left(x^{N}, \mathcal{C}^{L, N}\right)=\frac{1}{N} \inf _{\boldsymbol{y}^{L} \in \mathcal{C}^{L, N}} \sum_{\ell=1}^{L}\left(\alpha_{\ell} \rho\left(x^{N}, \boldsymbol{y}_{\ell}\right)+\beta_{\ell}\left|\boldsymbol{y}_{\ell}\right|\right)$

is achieved for every $x^{N} \in A^{N}$ and $\alpha^{L}, \beta^{L} \geq \mathbf{0}$. Further, the infima in the definitions of $j_{N}^{\mathrm{fr}}\left(\alpha^{L}, \beta^{L}, \mu\right)$ (12) and $j_{N}^{\mathrm{vr}}\left(\alpha^{L}, \beta^{L}, \mu\right)(14)$ may be restricted to quantizers $Q^{L, N} \in$ $\mathcal{Q}^{\text {fr }}(L, N)$ and $Q^{L, N} \in \mathcal{Q}^{\operatorname{vr}}(L, N)$, respectively, such that

$$
Q^{L, N}\left(x^{N}\right)=\arg \min _{\boldsymbol{y}^{L} \in \mathcal{C}^{L, N}} \sum_{\ell=1}^{L}\left(\alpha_{\ell} \rho\left(x^{N}, \boldsymbol{y}_{\ell}\right)+\beta_{\ell}\left|\boldsymbol{y}_{\ell}\right|\right)
$$

for all $x^{N} \in A^{N}$, where $\mathcal{C}^{L, N}$ is the codebook associated with $L$-resolution quantizer $Q^{L, N}$. Thus

$$
\begin{aligned}
& j_{N}^{\mathrm{fr}}\left(\alpha^{L}, \beta^{L}, \mu\right)=\inf _{\mathcal{C}^{L, N} \in \mathcal{K}^{\mathrm{fr}}(L, N)} d_{\left(\alpha^{L}, \beta^{L}\right)}\left(\mu, \mathcal{C}^{L, N}\right) \\
& j_{N}^{\mathrm{vr}}\left(\alpha^{L}, \beta^{L}, \mu\right)=\inf _{\mathcal{C}^{L, N} \in \mathcal{K}^{\mathrm{vr}}(L, N)} d_{\left(\alpha^{L}, \beta^{L}\right)}\left(\mu, \mathcal{C}^{L, N}\right)
\end{aligned}
$$

where

$$
d_{\left(\alpha^{L}, \beta^{L}\right)}\left(\mu, \mathcal{C}^{L, N}\right)=E_{\mu} d_{\left(\alpha^{L}, \beta^{L}\right)}\left(X^{N}, \mathcal{C}^{L, N}\right) .
$$

Similarly, for any $\mathcal{C}^{L, N} \in \mathcal{K}^{\mathrm{fr}, R^{L}}(L, N)$, the infimum

$d_{\left(\alpha^{L}, \beta^{L}\right)}^{R^{L}}\left(x^{N}, \mathcal{C}^{L, N}\right)=\frac{1}{N} \inf _{\boldsymbol{y}^{L} \in \mathcal{C}^{L, N}} \sum_{\ell=1}^{L}\left(\alpha_{\ell} \rho\left(x^{N}, \boldsymbol{y}_{\ell}\right)+\beta_{\ell} N R_{\ell}\right)$

is achieved for every $x^{N} \in A^{N}$ and $\alpha^{L}, \beta^{L} \geq \mathbf{0}$ and the infimum in the definition of $j_{N}^{\mathrm{fr}, R^{L}}\left(\alpha^{L}, \beta^{L}, \mu\right)$ (13) may be restricted to quantizers $Q^{L, N} \in \mathcal{Q}^{\mathrm{fr}, R^{L}}(L, N)$ such that

$Q^{L, N}\left(x^{N}\right)=\arg \min _{\boldsymbol{y}^{L} \in \mathcal{C}^{L, N}} \sum_{\ell=1}^{L}\left(\alpha_{\ell} \rho\left(x^{N}, \boldsymbol{y}_{\ell}\right)+\beta_{\ell} N R_{\ell}\right)$

for all $x^{N} \in A^{N}$, where $\mathcal{C}^{L, N}$ is the codebook associated with $L$-resolution quantizer $Q^{L, N}$. Thus

$$
j_{N}^{\mathrm{fr}, R^{L}}\left(\alpha^{L}, \beta^{L}, \mu\right)=\inf _{\mathcal{C}^{L, N} \in \mathcal{K}^{f \mathrm{r}, R^{L}}(L, N)} d_{\left(\alpha^{L}, \beta^{L}\right)}^{R^{L}}\left(\mu, \mathcal{C}^{L, N}\right)
$$

where

$$
d_{\left(\alpha^{L, \beta} \beta^{L}\right)}^{R^{L}}\left(\mu, \mathcal{C}^{L, N}\right)=E_{\mu} d_{\left(\alpha^{L}, \beta^{L}\right)}^{R^{L}}\left(X^{N}, \mathcal{C}^{L, N}\right) .
$$

Proof: The first part of the proof treats the achievability of the infima in (27) and (30). For any $\mathcal{C}^{L, N} \in \mathcal{Q}^{\text {fr }}(L, N)$, the result is immediate since fixed-rate codes use necessarily finite codebooks. Given any nonempty codebook $\mathcal{C}^{L, N} \in$ $\mathcal{K}^{\mathrm{vr}}(L, N)$, choose any $\boldsymbol{z}^{L} \in \mathcal{C}^{L, N}$. Let

$$
c=\sum_{\ell=1}^{L}\left(\alpha_{\ell} \rho\left(x^{N}, \boldsymbol{z}_{\ell}\right)+\beta_{\ell}\left|\boldsymbol{z}_{\ell}\right|\right) .
$$

For each $\ell \in\{1, \cdots, L\}$, successively define

$$
\begin{aligned}
\mathcal{S}_{\ell}= & \left\{\boldsymbol{y}_{\ell} \in \mathcal{C}_{(\ell)}^{N}\left(\boldsymbol{y}_{\ell-1}\right):\right. \\
& \left.\boldsymbol{y}_{\ell-1} \in \mathcal{S}_{\ell-1}, \alpha_{\ell} \rho\left(X^{N}, \boldsymbol{y}_{\ell}\right)+\beta_{\ell}\left|\boldsymbol{y}_{\ell}\right| \leq c\right\}
\end{aligned}
$$


where $\mathcal{S}_{0}=\left\{\boldsymbol{y}_{0}\right\}$ and $\mathcal{C}_{(1)}^{N}\left(\boldsymbol{y}_{0}\right)=\mathcal{C}_{(1)}^{N}$ by definition. By definition of $\mathcal{K}^{\operatorname{vr}}(L, N)$ and $\mathcal{Q}^{\operatorname{vr}}(L, N), \mathcal{C}^{L, N} \in \mathcal{K}^{\operatorname{vr}}(L, N)$ implies that the Kraft inequality holds for each codebook in $\left\{\mathcal{C}_{(\ell)}^{N}\left(\boldsymbol{y}_{\ell-1}\right): 1 \leq \ell \leq L, \boldsymbol{y}_{\ell-1} \in \mathcal{C}_{(\ell-1)}^{N}\right\}$. Thus $\left|\mathcal{S}_{1}\right|$ is finite, and, for any $2 \leq \ell \leq L$, the same argument and the fact that $\left|\mathcal{S}_{\ell-1}\right|$ is finite imply that $\left|\mathcal{S}_{\ell}\right|$ is finite. Notice, then, that the number of $L$-resolution codewords satisfying

$$
\sum_{\ell=1}^{L}\left(\alpha_{\ell} \rho\left(X^{N}, \boldsymbol{y}_{\ell}\right)+\beta_{\ell}\left|\boldsymbol{y}_{\ell}\right|\right) \leq c
$$

is less than or equal to $\prod_{\ell=1}^{L}\left|\mathcal{S}_{\ell}\right|$, which is finite. Since the infima in (27) and (30) are achieved, the quantizers defined in (28) and (31), called (modified) nearest neighbor fixed-rate, variable-rate, and fixed-rate- $R^{L}$ quantizers, exist.

Let $\mathcal{Q}_{\mathrm{NN}}^{\mathrm{vr}}(L, N) \subseteq \mathcal{Q}^{\mathrm{vr}}(L, N)$ be the set of nearest neighbor variable-rate quantizers associated with codebooks in $\mathcal{K}^{\mathrm{vr}}(L, N)$. Now consider any codebook $\mathcal{C}^{L, N}$ and any two quantizers $Q, Q^{\prime}$ using codebook $\mathcal{C}^{L, N}$ such that $Q \in \mathcal{Q}^{\operatorname{vr}}(L, N)$ and $Q^{\prime} \in \mathcal{Q}_{\mathrm{NN}}^{\mathrm{vr}}(L, N)$. Since $Q^{\prime}$ is a nearest neighbor quantizer

$$
\begin{aligned}
& \sum_{\ell=1}^{L}\left(\alpha_{\ell} \rho\left(x^{N}, Q_{(\ell)}\left(x^{N}\right)\right)+\beta_{\ell}\left|Q_{(\ell)}\left(x^{N}\right)\right|\right) \\
& \geq \sum_{\ell=1}^{L}\left(\alpha_{\ell} \rho\left(x^{N}, Q_{(\ell)}^{\prime}\left(x^{N}\right)\right)+\beta_{\ell}\left|Q_{(\ell)}^{\prime}\left(x^{N}\right)\right|\right)
\end{aligned}
$$

for any $x^{N} \in A^{N}$, and thus

$$
\begin{aligned}
& E_{\mu^{N}} \sum_{\ell=1}^{L}\left(\alpha_{\ell} \rho\left(X^{N}, Q_{(\ell)}\left(X^{N}\right)\right)+\beta_{\ell}\left|Q_{(\ell)}\left(X^{N}\right)\right|\right) \\
& \quad \geq E_{\mu^{N}} \sum_{\ell=1}^{L}\left(\alpha_{\ell} \rho\left(X^{N}, Q_{(\ell)}^{\prime}\left(X^{N}\right)\right)+\beta_{\ell}\left|Q_{(\ell)}^{\prime}\left(X^{N}\right)\right|\right) .
\end{aligned}
$$

As a result

$$
\begin{aligned}
& j_{N}^{\mathrm{vr}}\left(\alpha^{L}, \beta^{L}, \mu\right) \\
& =\frac{1}{N} \inf _{Q \in \mathcal{Q}^{\mathrm{vr}}(L, N)} E_{\mu^{N}} \sum_{\ell=1}^{L}\left(\alpha_{\ell} \rho\left(X^{N}, Q_{(\ell)}\left(X^{N}\right)\right)\right. \\
& \left.+\beta_{\ell}\left|Q_{(\ell)}\left(X^{N}\right)\right|\right) \\
& =\frac{1}{N} \inf _{Q^{\prime} \in \mathcal{Q}_{\mathrm{vv}}^{v \mathrm{v}}(L, N)} E_{\mu^{N}} \sum_{\ell=1}^{L}\left(\alpha_{\ell} \rho\left(X^{N}, Q_{(\ell)}^{\prime}\left(X^{N}\right)\right)\right. \\
& \left.+\beta_{\ell}\left|Q_{(\ell)}^{\prime}\left(X^{N}\right)\right|\right) \\
& =\frac{1}{N} \inf _{\mathcal{C}^{L, N} \in \mathcal{K}^{\mathrm{vr}}(L, N)} E_{\mu^{N}} \inf _{\boldsymbol{y}^{L} \in \mathcal{C}^{L, N}} \sum_{\ell=1}^{L}\left(\alpha_{\ell} \rho\left(X^{N}, \boldsymbol{y}_{\ell}\right)\right. \\
& =\inf _{\mathcal{C}^{L, N} \in \mathcal{K}^{\mathrm{vr}}(L, N)} E_{\mu^{N}} d_{\left(\alpha^{L}, \beta^{L}\right)}\left(X^{N}, \mathcal{C}^{L, N}\right) .
\end{aligned}
$$

The arguments for $j_{N}^{\mathrm{fr}}\left(\alpha^{L}, \beta^{L}, \mu\right)$ and $j_{N}^{\mathrm{fr},} R^{L}\left(\alpha^{L}, \beta^{L}, \mu\right)$ use the same approach.

\section{B. Stationary Ergodic Sources}

This section focuses on stationary ergodic sources. Lemma 9 demonstrates the achievability of the first-order weighted distortion-rate function. The proof relies upon a random coding argument paralleling the random coding argument for singleresolution source codes.

Lemma 9 (Achievability of $J_{1}^{R^{L}}\left(\alpha^{L}, \beta^{L}, \mu\right)$ and $J_{1}\left(\alpha^{L}\right.$, $\left.\beta^{L}, \mu\right)$ ): Suppose that $\mu$ is stationary and ergodic, $A$ is Polish, $\rho(x, y)$ is continuous in $x$ for each $y$, and there exists a reference letter $y_{1}^{*}$ such that $E_{\mu^{1}} \rho\left(X^{1}, y_{1}^{*}\right)<\infty$. Then for any $\alpha^{L}, \beta^{L} \geq \mathbf{0}$ such that $\sum_{\ell=1}^{L}\left(\alpha_{\ell}+\beta_{\ell}\right)=1$ :

- $J_{1}^{R^{L}}\left(\alpha^{L}, \beta^{L}, \mu\right)$ is achievable by fixed-rate- $R^{L}$ coding;

- $J_{1}\left(\alpha^{L}, \beta^{L}, \mu\right)$ is achievable by fixed-rate coding;

- $J_{1}\left(\alpha^{L}, \beta^{L}, \mu\right)$ is achievable by variable-rate coding.

Proof: The majority of the proof works toward demonstrating that for any $\epsilon>0$ there exists an integer $N \geq 1$ and a quantizer $Q^{L, N} \in \mathcal{Q}^{\mathrm{f}, R^{L}}(L, N)$ such that

$$
\begin{aligned}
\frac{1}{N}\left[\alpha^{L} \boldsymbol{D}^{t}(\mu,\right. & \left.\left.Q^{L, N}\right)+\beta^{L} \boldsymbol{R}^{t}\left(\mu, Q^{L, N}\right)\right] \\
& <\frac{1}{N}\left[\alpha^{L} \boldsymbol{D}^{t}\left(\mu, Q^{L, N}\right)+\beta^{L}\left(N R^{L}\right)^{t}\right] \\
& \leq J_{1}^{R^{L}}\left(\alpha^{L}, \beta^{L}, \mu\right)+\epsilon .
\end{aligned}
$$

This result implies that $J_{1}^{R^{L}}\left(\alpha^{L}, \beta^{L}, \mu\right)$ is achievable by fixedrate- $R^{L}$ coding and, since

$$
J_{1}\left(\alpha^{L}, \beta^{L}, \mu\right)=\inf _{R^{L}} J_{1}^{R^{L}}\left(\alpha^{L}, \beta^{L}, \mu\right)
$$

(20), $J_{1}\left(\alpha^{L}, \beta^{L}, \mu\right)$ is achievable by fixed-rate coding. The fact that $\mathcal{Q}^{\text {fr }}(L, N) \subseteq \mathcal{Q}^{\operatorname{vr}}(L, N)$ implies that $J_{1}\left(\alpha^{L}, \beta^{L}, \mu\right)$ is also achievable by variable-rate codes.

In pursuing the first result, Lemma 8 implies that it is sufficient to consider only nearest neighbor quantizers. The goal, then, is to design a codebook $\mathcal{C}^{L, N}$ in the class $\mathcal{K}^{\mathrm{fr}, R^{L}}(L, N)$ of fixed-rate- $R^{L}$, dimension- $N$ codebooks such that

$$
d_{\left(\alpha^{L}, \beta^{L}\right)}^{R^{L}}\left(\mu, \mathcal{C}^{L, N}\right) \leq J_{1}^{R^{L}}\left(\alpha^{L}, \beta^{L}, \mu\right)+\epsilon .
$$

The following random coding argument shows that the expected performance of a randomly drawn codebook is good, thereby proving the existence of a single codebook with performance at least as good as the expected performance of the randomly drawn codebook.

Consider any channel $q^{N}$ from $A^{N}$ to $\left(\hat{A}^{N}\right)^{L}$. Let $\nu^{N}$ be the marginal on $\left(\hat{A}^{N}\right)^{L}$ induced by $\mu^{N}$ and $q^{N}$. For any $1 \leq \ell \leq L$ and $\boldsymbol{y}^{\ell} \in\left(\hat{A}^{N}\right)^{\ell}$, the notation $\nu^{N}\left(\boldsymbol{y}^{\ell}\right)$ denotes the marginal distribution on $\boldsymbol{y}^{\ell}$ and $\nu^{N}\left(\boldsymbol{y}_{\ell} \mid \boldsymbol{y}^{\ell-1}\right)$ describes the conditional marginal on $\boldsymbol{y}_{\ell}$ given $\boldsymbol{y}^{\ell-1}$. Then for any $r^{L}<R^{L}$, design an $L$-resolution code as follows. Design the first-order codebook as

$\mathcal{C}_{(1)}^{N}=\left\{\boldsymbol{y}_{1}, \cdots, \boldsymbol{y}_{\left\lfloor 2^{N r_{1}}\right\rfloor-1}, \boldsymbol{y}^{*}\right\}$

s.t. $\boldsymbol{y}_{i} \in \hat{A}^{N}$ is drawn i.i.d. $\sim \nu^{N}(\boldsymbol{y}), \quad 1 \leq i \leq\left\lfloor 2^{n r_{1}}\right\rfloor-1$ 
where $y^{*}=\left(y_{1}^{*}, \cdots, y_{1}^{*}\right) \in \hat{A}^{N}$. For each $1<\ell \leq L$, successively define

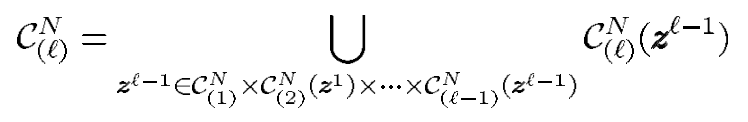

where

$\mathcal{C}_{(\ell)}^{N}\left(\boldsymbol{z}^{\ell-1}\right)=\left\{\boldsymbol{y}_{1}, \cdots, \boldsymbol{y}_{\left\lfloor 2^{\left.N r_{\ell}\right\rfloor-1}\right.}, \boldsymbol{y}^{*}\right\}$

s.t. $\boldsymbol{y}_{i} \in \hat{A}^{N}$ is drawn i.i.d. $\sim \nu^{N}\left(\boldsymbol{y} \mid \boldsymbol{z}^{\ell-1}\right), \quad 1 \leq i \leq 2^{n r_{\ell}}$.

(Notice that while arguments earlier in this work describe resolution- $\ell$ codebooks in terms of their resolution- $(\ell-1)$ predecessors, the current development describes a resolution- $\ell$ codebook in terms of all $\ell-1$ preceding resolutions. The two descriptions are equivalent.)

The above random code-design algorithm can be used to design a fixed-rate- $R^{L} L$-resolution source code for any test channel $q^{N}$. The discussion now turns to the choice of $q^{N}$. By definition of $J_{1}^{R^{L}}\left(\alpha^{L}, \beta^{L}, \mu\right)$, for any $\epsilon>0$ there exists a onedimensional test channel $q^{1}$ such that $I_{\mu^{1} q^{1}}\left(X ; Y_{\ell} \mid Y^{\ell-1}\right)<$ $R_{\ell}$ for each $\ell \in\{1, \cdots, L\}$ and

$$
\begin{aligned}
\sum_{\ell=1}^{L}\left(\alpha_{\ell} E_{\mu^{1} q^{1}} \rho\left(X^{1}, Y_{\ell}\right)+\beta_{\ell} I_{\mu^{1} q^{1}}(X ;\right. & \left.\left.Y_{\ell} \mid Y^{\ell-1}\right)\right) \\
& <J_{1}^{R^{L}}\left(\alpha^{L}, \beta^{L}, \mu\right)+\epsilon .
\end{aligned}
$$

Set $q^{N}$ to be the conditional probability measure corresponding to $N$ successive uses of test channel $q^{1}$. Then the facts that $\mu^{N}$ is stationary and ergodic and $q^{N}$ is memoryless together imply that $\mu^{N} q^{N}$ is stationary and ergodic [5, Theorem 7.2.1]. Further, since $J_{1}^{R^{L}}\left(\alpha^{L}, \beta^{L}, \mu\right)=\infty$ is trivially achieved, attention can here be restricted to $R_{\ell}$ finite for all $\ell \in$ $\{1, \cdots, L\}$, in which case $I_{\mu^{1} q^{1}}\left(X^{1} ; Y_{\ell} \mid Y^{\ell-1}\right)<R_{\ell}<$ $\infty$ for all $1 \leq \ell \leq L$, which implies $I_{\mu^{1} q^{1}}\left(X^{1} ; Y^{L}\right)<$ $\infty$. Finally, $(1 / N) I_{\mu^{N} q^{N}}\left(X^{N} ; \boldsymbol{Y}^{L}\right) \leq I_{\mu^{1} q^{1}}\left(X^{1} ; Y^{L}\right)<\infty$ (since dependence between the $N$ dimensions cannot increase the average mutual information), which implies $p^{N} \ll \pi^{N}$ and $f_{N}=d p^{N} / d \pi^{N}$ exists and is unique $p^{N}$-almost everywhere [5, Theorem 7.1.13].

Define $T_{\epsilon}^{N}$ as

$$
\begin{aligned}
T_{\epsilon}^{N}=\{ & \left(x^{N}, \boldsymbol{y}^{L}\right) \in A^{N} \times\left(\hat{A}^{N}\right)^{L}: \\
& \left|\frac{1}{N} \rho\left(x^{N}, \boldsymbol{y}_{\ell}\right)-E_{\mu^{1} q^{1}} \rho\left(X^{1}, Y_{\ell}\right)\right| \leq \epsilon \\
& \left|\frac{1}{N} \log f_{N}\left(x^{N} ; \boldsymbol{y}^{\ell}\right)-I_{\mu^{1} q^{1}}\left(X^{1} ; Y^{\ell}\right)\right| \leq \epsilon \\
& 1 \leq \ell \leq L\} .
\end{aligned}
$$

Further, for any $x^{N} \in A^{N}$, let

$$
T_{\epsilon}^{N}\left(x^{N}\right)=\left\{\boldsymbol{y}^{L}:\left(x^{N}, y^{L}\right) \in T_{\epsilon}^{N}\right\} .
$$

Then $\left(x^{N}, \boldsymbol{y}^{\ell}\right) \in T_{\epsilon}^{N}$ implies

$$
\begin{aligned}
\frac{d p^{N}\left(x^{N}, \boldsymbol{y}^{\ell}\right)}{d \mu^{N}\left(x^{N}\right)} & 2^{-N\left(I_{\mu} 1_{q}\left(X^{1} ; Y^{\ell}\right)+\epsilon\right)} \\
& \leq d \nu\left(\boldsymbol{y}^{\ell}\right) \\
& \leq \frac{d p^{N}\left(x^{N}, \boldsymbol{y}^{\ell}\right)}{d \mu^{N}\left(x^{N}\right)} 2^{-N\left(I_{\mu} q_{q}\left(X^{1} ; Y^{\ell}\right)-\epsilon\right)}
\end{aligned}
$$

$$
\begin{aligned}
& \text { for all } 1 \leq \ell \leq L \text { and, combining the } \ell \text { th and }(\ell-1) \text { th term } \\
& \begin{aligned}
& \frac{d p^{N}\left(x^{N}, \boldsymbol{y}^{\ell}\right)}{d p^{N}\left(x^{N}, \boldsymbol{y}^{\ell-1}\right)} 2^{-N\left(I_{\mu 1} q^{1}\left(X^{1} ; Y_{\ell} \mid Y^{\ell-1}\right)+2 \epsilon\right)} \\
& \leq d \nu\left(\boldsymbol{y}_{\ell} \mid \boldsymbol{y}^{\ell-1}\right) \\
& \leq \frac{d p^{N}\left(x^{N}, \boldsymbol{y}^{\ell}\right)}{d p^{N}\left(x^{N}, \boldsymbol{y}^{\ell-1}\right)} 2^{-N\left(I_{\mu^{1} q^{1}}\left(X^{1} ; Y_{\ell} \mid Y^{\ell-1}\right)-2 \epsilon\right)}
\end{aligned}
\end{aligned}
$$

Given these definitions, the expected performance of the above random code is

$$
\begin{aligned}
& E d_{\left(\alpha^{L}, \beta^{L}\right)}^{R^{L}}\left(\mu, \mathcal{C}^{L, N}\right) \\
& =E E_{\mu^{N}} \inf _{\boldsymbol{y}^{L} \in \mathcal{C}^{L, N}} \frac{1}{N} \sum_{\ell=1}^{L}\left(\alpha_{\ell} \rho\left(X^{N}, \boldsymbol{y}_{\ell}\right)+\beta_{\ell} N R_{\ell}\right) \\
& \leq \operatorname{Pr}\left(\mathcal{C}^{L, N} \cap T_{\epsilon}^{N}\left(X^{N}\right) \neq \emptyset\right) \frac{1}{N} \\
& \times \sum_{\ell=1}^{L}\left(\alpha_{\ell}\left(E_{\mu^{N} q^{N}} \rho\left(X^{N}, Y_{\ell}\right)+\epsilon\right)+\beta_{\ell} N R_{\ell}\right) \\
& +\operatorname{Pr}\left(\mathcal{C}^{L, N} \cap T_{\epsilon}^{N}\left(X^{N}\right)=\emptyset\right) \frac{1}{N} \sum_{\ell=1}^{L}\left(\alpha_{\ell} N d^{*}+\beta_{\ell} N R_{\ell}\right) \\
& \leq \sum_{\ell=1}^{L}\left(\frac{1}{N} \alpha_{\ell} E_{\mu^{N} q^{N}} \rho\left(X^{N}, \boldsymbol{Y}_{\ell}\right)+\beta_{\ell} N R_{\ell}\right) \\
& +\epsilon+\operatorname{Pr}\left(\mathcal{C}^{L, N} \cap T_{\epsilon}^{N}\left(X^{N}\right)=\emptyset\right) d^{*} .
\end{aligned}
$$

Setting $k_{\ell}=\left\lfloor 2^{N R_{\ell}}\right\rfloor-1$, the probability

$$
\operatorname{Pr}\left(\mathcal{C}^{L, N} \cap T_{\epsilon}^{N}\left(X^{N}\right)=\emptyset\right)
$$

is bounded by giving its explicit equation and then alternately applying the inequalities $(1-x y)^{n} \leq 1-x+e^{-y n}$ for any $0 \leq x, y \leq 1$ [25, Theorem 13.5.3] (or its consequence $(1-x y+z)^{n} \leq(1-x)(1+z)^{n}+\left(z+e^{-y /(1+z)}\right)^{n}$ for any $z \geq 0$ ) and (35). This approach gives

$$
\begin{aligned}
& \operatorname{Pr}\left(\mathcal{C}^{L, N} \cap T_{\epsilon}^{N}\left(X^{N}\right)=\emptyset\right) \\
& \leq \int\left[\int \cdots \left[\int \left[\int 1\left(\left(x^{N}, \boldsymbol{y}^{L}\right) \notin T_{\epsilon}^{N}\right)\right.\right.\right. \\
& \left.\left.\times d \nu\left(y_{L} \mid y^{L-1}\right)\right]^{k_{L}} d \nu\left(y_{L-1} \mid y^{L-2}\right)\right]^{k_{L-1}} \\
& \left.\cdots d \nu\left(\boldsymbol{y}_{1}\right)\right]^{k_{1}} d \mu\left(x^{N}\right) \\
& =\int\left[\int \cdots \left[\int \left[1-\int 1\left(\left(x^{N}, y^{L}\right) \in T_{\epsilon}^{N}\right)\right.\right.\right. \\
& \left.\left.\times d \nu\left(\boldsymbol{y}_{L} \mid \boldsymbol{y}^{L-1}\right)\right]^{k_{L}} d \nu\left(\boldsymbol{y}_{L-1} \mid y^{L-2}\right)\right]^{k_{L-1}} \\
& \left.\cdots d \nu\left(y_{1}\right)\right]^{k_{1}} d \mu\left(x^{N}\right)
\end{aligned}
$$




$$
\begin{aligned}
& \leq \int\left[\int \cdots \left[\int \left[1-\int 1\left(\left(x^{N}, y^{L}\right) \in T_{\epsilon}^{N}\right)\right.\right.\right. \\
& \left.\times d p^{N}\left(\boldsymbol{y}_{L} \mid x^{N}, \boldsymbol{y}^{L-1}\right) 2^{-N\left(I_{\mu 1} q^{1}\left(X^{1} ; Y_{L} \mid Y^{L-1}\right)+2 \epsilon\right)}\right]^{k_{L}} \\
& \left.\left.\times d \nu\left(y_{L-1} \mid \boldsymbol{y}^{L-2}\right)\right]^{k_{L-1}} \cdots d \nu\left(y_{1}\right)\right]^{k_{1}} d \mu\left(x^{N}\right) \\
& \leq \int\left[\int \cdots \left[\int \left[1-\int 1\left(\left(x^{N}, \boldsymbol{y}^{L}\right) \in T_{\epsilon}^{N}\right)\right.\right.\right. \\
& \left.\times d p^{N}\left(\boldsymbol{y}_{L} \mid x^{N}, \boldsymbol{y}^{L-1}\right)+e^{-k_{L} 2^{-N\left(I_{\mu} q_{q}\left(X^{1} ; Y_{L} \mid Y^{L-1}\right)+2 c\right)}}\right] \\
& \left.\left.\times d \nu\left(\boldsymbol{y}_{L-1} \mid \boldsymbol{y}^{L-2}\right)\right]^{k_{L-1}} \cdots d \nu\left(\boldsymbol{y}_{1}\right)\right]^{k_{1}} d \mu\left(x^{N}\right) \\
& \leq \int\left[\int \cdots \left[1-\iint 1\left(\left(x^{N}, y^{L}\right) \in T_{\epsilon}^{N}\right)\right.\right. \\
& \times d p^{N}\left(\boldsymbol{y}_{L-1}, \boldsymbol{y}_{L} \mid x^{N}, \boldsymbol{y}^{L-2}\right) 2^{-N\left(I_{\mu} 1_{q} 1\left(X^{1} ; Y_{L-1} \mid Y^{L-2}\right)+2 \epsilon\right)} \\
& \left.+e^{-k_{L} 2^{-N\left(I_{\mu 1} 1^{1}\left(X^{1} ; Y_{L} \mid Y^{L-1}\right)+2 c\right)}}\right]^{k_{L-1}} \\
& \left.\cdots d \nu\left(\boldsymbol{y}_{1}\right)\right]^{k_{1}} d \mu\left(x^{N}\right)
\end{aligned}
$$$$
\leq \cdots
$$$$
\leq c \epsilon
$$

for some finite constant $c$ for $N$ large enough if

$$
r_{\ell}=I_{\mu^{1} q^{1}}\left(X^{1} ; Y_{\ell} \mid Y^{\ell-1}\right)+4 \epsilon
$$

for all $\ell \in\{1, \cdots, L\}$. The above result relies in its final step on the generalized Asymptotic Equipartition Property (AEP) for stationary ergodic sources [5, Corollary 7.3.1]. Thus

$$
\begin{aligned}
E d_{\left(\alpha^{L}, \beta^{L}\right)}^{R^{L}}\left(\mu, \mathcal{C}^{L, N}\right) & \leq \sum_{\ell=1}^{L}\left(\alpha_{\ell} E_{\mu^{1} q^{1}} \rho\left(X^{1}, Y_{\ell}\right)+\beta_{\ell} R_{\ell}\right)+c^{\prime} \epsilon \\
& <J_{1}^{R^{L}}\left(\alpha^{L}, \beta^{L}, \mu\right)+\left(c^{\prime}+1\right) \epsilon
\end{aligned}
$$

for some finite constant $d^{\prime}$ and arbitrary $\epsilon>0$.

Lemma 9 establishes the achievability of the firstorder weighted distortion-rate functions $J_{1}^{R^{L}}\left(\alpha^{L}, \beta^{L}, \mu\right)$ and $J_{1}\left(\alpha^{L}, \beta^{L}, \mu\right)$, but does not address the achievability of higher order distortion-rate functions. Next consider the achievability of these higher order functions. In doing so, it is tempting to think that blocking the source into $N$-dimensional vectors and repeating the above proof on the resulting blocked source will yield the desired results. Unfortunately, this technique fails because the generalized AEP requires source ergodicity, and while the original source is assumed ergodic, ergodicity of the original source does not imply ergodicity of the blocked source. The following argument takes a similar approach to that used in [5] for decomposing the blocked source into its ergodic modes and then building a code for the nonergodic source by combining codes from the ergodic components.
The following notation, from [5], is useful in completing the argument outlined above. Let $x \leftrightarrow x^{\prime}$ denote the relationship between $x=\left(x_{1}, x_{2}, \cdots\right)$ and $x^{\prime}=\left(x_{1}^{n}, x_{n+1}^{2 n}, \cdots\right)$. Similarly, for any set $F \in \mathcal{A}$, let $F \leftrightarrow F^{\prime}$ express the relationship

$$
F^{\prime}=\left\{x^{\prime} \in A^{\prime}: x \leftrightarrow x^{\prime} \text { for some } x \in F\right\} ;
$$

use $\mathcal{A} \leftrightarrow \mathcal{A}^{\prime}$; to mean

$$
\mathcal{A}^{\prime}=\left\{F^{\prime}: F \leftrightarrow F^{\prime} \text { for some } F \in \mathcal{A}\right\}
$$

define $\mu \leftrightarrow \mu^{\prime}$ such that $\mu(F)=\mu^{\prime}\left(F^{\prime}\right)$; and finally use the shift operator $T^{\prime}$ as $T^{\prime} x^{\prime}=\left(T^{n} x\right)^{\prime}$. Thus the source $\left(A^{\prime}, \mu^{\prime}, \mathcal{A}^{\prime}, T^{\prime}\right)$, henceforth denoted $\left[A^{\prime}, \mu^{\prime}\right]$, is a version of $[A, \mu]$ broken into $n$-dimensional vectors.

As mentioned previously, ergodicity of $[A, \mu]$ with respect to $T$ does not imply ergodicity of $\left[A^{\prime}, \mu^{\prime}\right]$ with respect to $T^{\prime}$. However, the following lemma establishes the existence of a finite ergodic decomposition for $\left[A^{\prime}, \mu^{\prime}\right]$ under the above conditions.

Lemma 10 (Ergodic Decomposition of $\left[A^{\prime}, \mu^{\prime}\right][5$, Theorem 7.2.3]): Consider a time-discrete, stationary, ergodic source $[A, \mu]$. The source $\left[A^{\prime}, \mu^{\prime}\right]$ created by blocking $[A, \mu]$ into $n$-dimensional vectors can be decomposed into $m$ ergodic modes $E_{1}^{\prime}, \cdots, E_{m}^{\prime}$ where $n=k m$ for some integer $k \geq 1$ and $E_{(i+j) \bmod m}=E_{i} T^{j}$ for all $1 \leq i \leq m, j \geq 0$. If $1 \leq i, j \leq m$, and $i \neq j$, then $\mu^{\prime}\left(E_{j}^{\prime}\right)=1 / m$ and $\mu^{\prime}\left(E_{i}^{\prime} \cap E_{j}^{\prime}\right)=0$.

While Lemma 9 does not apply to source $\left[A^{\prime}, \mu^{\prime}\right]$ (since $\left[A^{\prime}, \mu^{\prime}\right]$ is not necessarily ergodic), Lemma 9 does apply to the ergodic components of $\left[A^{\prime}, \mu^{\prime}\right]$, described in Lemma 10. Thus the approach taken is to design a distinct fixed-rate code for each ergodic component and combine those codes to yield a single code that achieves, to arbitrary accuracy, the $n$ th-order weighted distortion-rate function. Achieving this result for arbitrary $n$ gets us arbitrarily close to the weighted distortion-rate function.

Lemma 11 (Achievability of $J^{R^{L}}\left(\alpha^{L}, \beta^{L}, \mu\right)$ and $J\left(\alpha^{L}\right.$, $\left.\left.\beta^{L}, \mu\right)\right)$ : Suppose that source $\mu$ is stationary and ergodic, $A$ is Polish, $\rho(x, y)$ is continuous in $x$ for each $y$, and there exists a reference letter $y_{1}^{*}$ such that $E_{\mu^{1}} \rho\left(X^{1}, y_{1}^{*}\right)<\infty$. Then for any $\alpha^{L}, \beta^{L} \geq \mathbf{0}$ such that $\sum_{\ell}\left(\alpha_{\ell}+\beta_{\ell}\right)=1$

- $J^{R^{L}}\left(\alpha^{L}, \beta^{L}, \mu\right)$ is achievable by fixed-rate- $R^{L}$ coding;

- $J\left(\alpha^{L}, \beta^{L}, \mu\right)$ is achievable by fixed-rate coding;

- $J\left(\alpha^{L}, \beta^{L}, \mu\right)$ is achievable by variable-rate coding.

Proof: The majority of the proof works toward showing that for any $\epsilon>0$ there exists an $N \geq 1$ and a quantizer $Q^{L, N} \in \mathcal{Q}^{\mathrm{fr}, R^{L}}(L, N)$ such that

$$
\begin{aligned}
\frac{1}{N}\left[\alpha^{L}\right. & \left.\boldsymbol{D}^{t}\left(\mu, Q^{L, N}\right)+\beta^{L} \boldsymbol{R}^{t}\left(\mu, Q^{L, N}\right)\right] \\
& <\frac{1}{N}\left[\alpha^{L} \boldsymbol{D}^{t}\left(\mu, Q^{L, N}\right)+\beta^{L}\left(N R^{L}\right)^{t}\right] \\
& \leq J^{R^{L}}\left(\alpha^{L}, \beta^{L}, \mu\right)+\epsilon
\end{aligned}
$$

which gives the achievability result for fixed-rate- $R^{L}$ source codes. Then taking the infimum over all $R^{L}$ gives the second 
result and the fact that $\mathcal{Q}^{\mathrm{fr}}(L, N) \subseteq \mathcal{Q}^{\mathrm{vr}}(L, N)$ implies the corresponding result for variable-rate codes. To prove the first result, fix a vector dimension $n>1$ and define $\left[A^{\prime}, \mu^{\prime}\right]$ to be the source created by blocking $[A, \mu]$ into $n$-vectors. Using Lemma 10 , decompose $\left[A^{\prime}, \mu^{\prime}\right]$ into $m$ ergodic modes $E_{1}^{\prime}, \cdots, E_{m}^{\prime}$. Thus the source $\left[A^{\prime}, \mu^{\prime}\right]$ may be viewed as a mixture of $m$ sources $\left[A^{\prime}, \mu_{i}^{\prime}\right]$ defined by

$$
\mu_{i}^{\prime}\left(F^{\prime}\right)=m \mu^{\prime}\left(F^{\prime} \cap E_{i}^{\prime}\right)
$$

for all $1 \leq i \leq m$ and $F^{\prime} \in \mathcal{A}^{\prime}$. By Lemma $10,\left[A^{\prime}, \mu_{i}^{\prime}\right]$ is ergodic with respect to $T^{\prime}$. For each $1 \leq i \leq m$, define $\mu_{i}$ such that for any $F \in \mathcal{A}, \mu_{i}(F)=m \mu\left(F \cap E_{i}\right)$ where $E_{i} \leftrightarrow E_{i}^{\prime}$. Notice that $\mu_{i}$ may not be ergodic with respect to $T$ even though $\mu_{i}^{\prime}$ is ergodic with respect to $T^{\prime}$. Notice further that

$$
\mu^{n}(F)=(1 / m) \sum_{i=1}^{m} \mu_{i}^{n}(F)
$$

for any $F \in \mathcal{A}^{n}$.

By Lemma 9, the ergodicity of $\mu_{i}^{\prime}$ for each $1 \leq i \leq m$ implies that for sufficiently large $N$ there exist blocklength$N$, fixed-rate- $n R^{L}$ codes for $\left[A^{\prime}, \mu_{i}^{\prime}\right]$ (or, equivalently, blocklength- $(N n)$, fixed-rate- $R^{L}$ codes for $\left.\left[A, \mu_{i}\right]\right)$ with performance per $n$-vector arbitrarily close to $J_{1}^{n R^{L}}\left(\alpha^{L}, \beta^{L}, \mu_{i}^{\prime}\right)$. Thus $m<\infty$ implies that for any $\epsilon>0$ and $N$ sufficiently large, there exists a collection of $m N$-dimensional codebooks

$$
\left\{\mathcal{C}^{L, N}(i): 1 \leq i \leq m\right\} \subseteq \mathcal{K}^{\prime} \mathrm{fr}, n R^{L}(L, N)
$$

such that

$$
d_{\left(\alpha^{L}, \beta^{L}\right)}^{n R^{L}}\left(\mu_{i}^{\prime}, \mathcal{C}^{L, N}(i)\right) \leq J_{1}^{n R^{L}}\left(\alpha^{L}, \beta^{L}, \mu_{i}^{\prime}\right)+\epsilon
$$

for each $1 \leq i \leq m$. Choose any such $N$ and let $R^{L}(i)=$ $\left(R_{1}(i), \cdots, R_{L}(i)\right)$ be the rate per vector associated with fixed-rate code $\mathcal{C}^{L, N}(i)$. Notice that by the definition of $\mathcal{K}^{\prime} \mathrm{fr}, n R^{L}(L, N), R_{\ell}(i)<n R_{\ell}$ for each $i \in\{1, \cdots, m\}$ and each $\ell \in\{1, \cdots, L\}$. Next, construct an $m(N n+1)$ dimensional codebook $\mathcal{C}^{L, m(N n+1)}$ for $\mu$ by concatenating together an ordered sequence of codewords from the $m$ codebooks described above and separating those codewords with escape characters. In particular

$$
\begin{aligned}
\mathcal{C}_{(1)}^{m(N n+1)} & \left\{\left(\boldsymbol{y}_{1} y_{1}^{*} \boldsymbol{y}_{2} y_{1}^{*} \cdots \boldsymbol{y}_{m} y_{1}^{*},\lceil\log m\rceil+N \sum_{i=1}^{m} R_{1}(i)\right):\right. \\
& \left.\exists j \geq 0 \text { s.t. } \boldsymbol{y}_{i} \in \mathcal{C}_{(1)}^{\prime N}\left((i+j)_{m}\right) \forall 1 \leq i \leq m\right\}
\end{aligned}
$$

where $(x)_{m}=x \bmod m$. For any $z^{m(N n+1)} \in \mathcal{C}_{(1)}^{m(N n+1)}$, let $j\left(z^{m(N n+1)}\right)$ be the unique integer $j \in\{0, \cdots, m-1\}$ such that

$$
z^{m(N n+1)}=\left(\boldsymbol{y}_{1} y_{1}^{*} \boldsymbol{y}_{2} y_{1}^{*} \cdots \boldsymbol{y}_{m} y_{1}^{*}\right)
$$

implies $\boldsymbol{y}_{i} \in \mathcal{C}_{(1)}^{\prime N}\left((i+j)_{m}\right)$. Then for each $1<\ell \leq L$, let

$$
\mathcal{C}_{(\ell)}^{m(N n+1)}=\bigcup_{z^{m(N n+1)} \in \mathcal{C}_{(\ell-1)}^{m(N n+1)}} \mathcal{C}_{(\ell)}^{m(N n+1)}\left(z^{m(N n+1)}\right)
$$

where for any $z^{m(N n+1)} \in \mathcal{C}_{(\ell-1)}^{m(N n+1)}$

$$
\begin{aligned}
\mathcal{C}_{(\ell)}^{m(N n+1)}\left(z^{m(N n+1)}\right) & \\
= & \left\{\left(\boldsymbol{y}_{1} y_{1}^{*} \boldsymbol{y}_{2} y_{1}^{*} \cdots \boldsymbol{y}_{m} y_{1}^{*}, N \sum_{i=1}^{m} R_{\ell}(i)\right)\right. \\
& \boldsymbol{y}_{i} \in \mathcal{C}_{(\ell)}^{\prime N}\left(\left(i+j\left(z^{m(N n+1)}\right)\right)_{m}\right)\left(z_{(i-1)(N n+1)+1}^{i(N n+1)}\right) \\
& \forall 1 \leq i \leq m\} .
\end{aligned}
$$

The resolution- 1 codebook $\mathcal{C}_{(1)}^{m(N n+1)}$ has size

$$
\left|\mathcal{C}_{(1)}^{m(N n+1)}\right|=m \prod_{i=1}^{m} 2^{N R_{1}(i)}
$$

and thus

$$
\begin{aligned}
& \sum_{\boldsymbol{y} \in \mathcal{C}_{(1)}^{m(N n+1)}} 2^{-|\boldsymbol{y}|} \\
& =\left(m \prod_{i=1}^{m} 2^{N R_{1}(i)}\right) 2^{-\left(\lceil\log m\rceil+N \sum_{i=1}^{m} R_{1}(i)\right)} \leq 1
\end{aligned}
$$

which shows that the Kraft inequality is satisfied in the first-resolution as required. For any $1 \leq \ell \leq L$ and any $z^{m(N n+1)} \in \mathcal{C}_{(\ell-1)}^{m(N n+1)}$, the resolution- $\ell$ codebook corresponding to $z^{m(N n+1)}$ has size

$$
\left|\mathcal{C}_{(\ell)}^{m(N n+1)}\left(z^{m(N n+1)}\right)\right|=\prod_{i=1}^{m} 2^{N R_{\ell}(i)}
$$

and thus

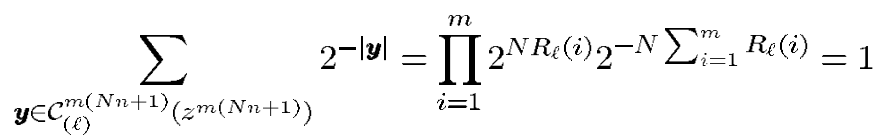

shows that Kraft's inequality is satisfied with equality in the $\ell$ th resolution of this fixed-rate, multiresolution code.

The rate per symbol in the code's first resolution is bounded as

$$
\frac{\lceil\log m\rceil+N \sum_{i=1}^{m} R_{1}(i)}{m(N n+1)} \leq \frac{1}{m n} \sum_{i=1}^{m} R_{1}(i)+\frac{\lceil\log m\rceil}{m(N n+1)}<R_{1}
$$

for $N$ large enough since $R_{1}(i)<n R_{1}$ for all $i$ implies $\frac{1}{m n} \sum_{i=1}^{m} R_{1}(i)<R_{1}$ and $m$ is constant since $n$ is fixed. The rate in the code's $\ell$ th resolution for any $1<\ell \leq L$ is given by

$$
\frac{N \sum_{i=1}^{m} R_{\ell}(i)}{m(N n+1)} \leq \frac{1}{m n} \sum_{i=1}^{m} R_{\ell}(i)<R_{\ell} .
$$

From Lemma $10, T^{-j} E_{(i+j)_{m}}=E_{i}$ and $n$ is an integer multiple of $m$ together imply that $\mu_{i}$ induces on the source letters in positions $k(N n+1)+1$ through $(k+1)(N n+1)$ the same probability measure that $\mu_{(i+k)_{m}}$ induces on positions 1 
through $N n+1$. Thus for each $1 \leq i \leq m$

$$
\begin{aligned}
& d_{\left(\alpha^{L}, \beta^{L}\right)}^{R^{L}}\left(\mu_{i}, \mathcal{C}^{L, m(N n+1)}\right) \\
& \quad \leq \frac{1}{m(N n+1)}\left[\sum_{j=1}^{m} N d_{\left(\alpha^{L}, \beta^{L}\right)}^{n R^{L}}\left(\mu_{j}^{\prime}, \mathcal{C}^{L, N}(j)\right)+m d^{*}\right] \\
& \quad \leq \frac{1}{m n} \sum_{j=1}^{m}\left[d_{\left(\alpha^{L}, \beta^{L}\right)}^{n R^{L}}\left(\mu_{j}^{\prime}, \mathcal{C}^{L, N}(j)\right)+n \epsilon\right] \\
& \quad \leq \frac{1}{m n} \sum_{j=1}^{m}\left[J_{1}^{n R^{L}}\left(\alpha^{L}, \beta^{L}, \mu_{j}^{\prime}\right)+(n+1) \epsilon\right] \\
& \quad \leq \frac{1}{m} \sum_{j=1}^{m}\left[J_{n}^{R^{L}}\left(\alpha^{L}, \beta^{L}, \mu_{j}\right)+2 \epsilon\right]
\end{aligned}
$$

for $N$ large enough since $m \leq n$ is constant, $\sum_{\ell=1}^{L} \alpha_{\ell} \leq 1$, $\sum_{\ell=1}^{L} \beta_{\ell} \leq 1, d^{*}<\infty$, and

$$
J_{1}^{n R^{L}}\left(\alpha^{L}, \beta^{L}, \mu_{j}^{\prime}\right)=n J_{n}^{R^{L}}\left(\alpha^{L}, \beta^{L}, \mu_{j}\right) .
$$

Thus

$$
\begin{aligned}
d_{\left(\alpha^{L}, \beta^{L}\right)}^{R^{L}}\left(\mu, \mathcal{C}^{L, m(N n+1)}\right) & =\frac{1}{m} \sum_{i=1}^{m} d_{\left(\alpha^{L}, \beta^{L}\right)}^{R^{L}}\left(\mu_{i}, \mathcal{C}^{L, m(N n+1)}\right) \\
& \leq \frac{1}{m} \sum_{j=1}^{m} J_{n}^{R^{L}}\left(\alpha^{L}, \beta^{L}, \mu_{j}\right)+2 \epsilon .
\end{aligned}
$$

From the definition of $J_{n}^{R^{L}}\left(\alpha^{L}, \beta^{L}, \mu\right)$ found in (18), for any $\epsilon>0$ there exists a test channel $q^{n}$ such that

$$
\frac{1}{n} I_{\mu^{n} q^{n}}\left(X^{n} ; Y_{\ell} \mid Y^{\ell-1}\right)<R_{\ell}
$$

for each $\ell \in\{1, \cdots, L\}$ and

$\frac{1}{n} \sum_{\ell=1}^{L}\left(\alpha_{\ell} E_{\mu^{n} q^{n}} \rho\left(X^{n}, \boldsymbol{Y}_{\ell}\right)+\beta_{\ell} n R_{\ell}\right)<J_{n}^{R^{L}}\left(\alpha^{L}, \beta^{L}, \mu\right)+\epsilon$.

Notice that $I_{\mu q}(X ; Y)$ concave in $\mu$ for fixed $q$ (e.g., [25, Theorem 2.7.4]) implies

$$
\begin{aligned}
\frac{1}{m} \sum_{i=1}^{m} I_{\mu_{i}^{n} q^{n}}\left(X^{n} ; \boldsymbol{Y}_{\ell} \mid \boldsymbol{Y}^{\ell-1}\right) & \leq I_{\mu^{n} q^{n}}\left(X^{n} ; \boldsymbol{Y}_{\ell} \mid \boldsymbol{Y}^{\ell-1}\right) \\
& <n R_{\ell}
\end{aligned}
$$

for each $1 \leq \ell \leq L$. Further, by definition of $\left\{\mu_{i}\right\}$

$$
\sum_{i=1}^{m} \frac{1}{m} E_{\mu_{i}^{n} q^{n}} \rho\left(X^{n}, \boldsymbol{Y}_{\ell}\right)=E_{\mu^{n} q^{n}} \rho\left(X^{n}, \boldsymbol{Y}_{\ell}\right)
$$

for each $1 \leq \ell \leq L$. Thus

$$
\begin{aligned}
\frac{1}{m} \sum_{i=1}^{m} J_{n}^{R^{L}}\left(\alpha^{L}, \beta^{L}, \mu_{i}\right) & \\
\leq & \frac{1}{m n} \sum_{i=1}^{m} \sum_{\ell=1}^{L}\left(\alpha_{\ell} E_{\mu_{i}^{n} q^{n}} \rho\left(X^{n}, \boldsymbol{Y}_{\ell}\right)+\beta_{\ell} n R_{\ell}\right) \\
& <J_{n}^{R^{L}}\left(\alpha^{L}, \beta^{L}, \mu\right)+\epsilon
\end{aligned}
$$

for arbitrary $\epsilon$, which implies

$$
\begin{aligned}
d_{\left(\alpha^{L}, \beta^{L}\right)}^{R^{L}}\left(\mu, \mathcal{C}^{L, m(N n+1)}\right) & \leq \sum_{i=1}^{m} \frac{1}{m} J_{n}^{R^{L}}\left(\alpha^{L}, \beta^{L}, \mu_{i}\right) \\
& \leq J_{n}^{R^{L}}\left(\alpha^{L}, \beta^{L}, \mu\right) .
\end{aligned}
$$

This is the desired result.

Lemmas 11 and 7 together yield Theorems 1 and 3.

\section{Stationary Nonergodic Sources}

The remainder of this paper treats the case of stationary nonergodic sources. Notice that the approach used in Lemma 11 to code for the nonergodic source $\left[A^{\prime}, \mu^{\prime}\right]$ made by blocking $[A, \mu]$ into $n$-blocks must here be modified to achieve coding results for general nonergodic sources. This modification is necessary since the properties relating the ergodic modes of $\left[A^{\prime}, \mu^{\prime}\right]$ (described in Lemma 10) do not hold for general nonergodic sources. The proofs given in this section rely on the functional ergodic decomposition developed in [21]. The following lemma describes conditions under which the functional ergodic decomposition holds.

Lemma 12 (Functional Ergodic Decomposition [21, Theorem 8.9.1]): Consider the dynamical system $\left(A^{\infty}, \mathcal{B}^{\infty}, \mu, T\right)$ with standard measurable space $\left(A^{\infty}, \mathcal{B}^{\infty}\right)$ and stationary measure $\mu$. Let $\left\{\mu_{x}: x \in A^{\infty}\right\}$ denote the ergodic decomposition. Let $f: \mathcal{P}_{s} \rightarrow \mathbb{R}^{+}$be a nonnegative functional defined for all stationary measures and satisfying the following properties:

1) $f\left(\mu_{x}\right)$ is $\mu$-integrable;

2) $f$ is an affine function of $\mu \in \mathcal{P}_{s}$; and

3) $f$ is upper semicontinuous; that is, if $\mu_{n}$ is a sequence of stationary measures converging to a stationary measure $\mu$ in the sense that $\mu_{n}(G) \rightarrow \mu(G)$ for all $G$ in a standard generating field for $\mathcal{B}^{\infty}$, then $f(\mu) \geq \lim \sup _{n} f\left(\mu_{n}\right)$.

Then

$$
f(\mu)=\int f\left(\mu_{x}\right) d \mu(x) .
$$

Application of Lemma 12, with $f(\mu)=j^{\mathrm{fr}, R^{L}}\left(\alpha^{L}, \beta^{L}, \mu\right)$ and $f(\mu)=j^{\mathrm{vr}}\left(\alpha^{L}, \beta^{L}, \mu\right)$ yields Theorems 5 and 7. Lemmas 13-15 work toward showing that the above functionals are affine. Lemmas 16 and 17 together establish that $j^{\mathrm{fr}, R^{L}}\left(\alpha^{L}, \beta^{L}, \mu\right)$ and $j^{\mathrm{vr}}\left(\alpha^{L}, \beta^{L}, \mu\right)$ are upper semicontinuous. The arguments parallel proofs from [26] and [6] for single-resolution fixed- and variable-rate lossy source codes, respectively.

Lemma 13 demonstrates that $j_{N}^{\mathrm{fr}, R^{L}}\left(\alpha^{L}, \beta^{L}, \mu\right)$ and $j_{N}^{\mathrm{vr}}\left(\alpha^{L}, \beta^{L}, \mu\right)$ are concave, but not too concave, in $\mu$.

Lemma 13: For any two sources $\mu_{1}$ and $\mu_{2}$, any $\lambda \in[0,1]$, any $\alpha^{L}, \beta^{L} \geq \mathbf{0}$, and any $N$

$$
\begin{aligned}
& j_{N}^{\mathrm{fr},} R^{L}\left(\alpha^{L}, \beta^{L}, \lambda \mu_{1}+(1-\lambda) \mu_{2}\right) \\
& \quad \geq \lambda j_{N}^{\mathrm{fr}, R^{L}}\left(\alpha^{L}, \beta^{L}, \mu_{1}\right)+(1-\lambda) j_{N}^{\mathrm{fr}, R^{L}}\left(\alpha^{L}, \beta^{L}, \mu_{2}\right) \\
& j_{N}^{\mathrm{fr},} R^{L}\left(\alpha^{L}, \beta^{L}, \lambda \mu_{1}+(1-\lambda) \mu_{2}\right) \\
& \quad \leq \lambda j_{N-k}^{\mathrm{fr}, R^{L}}\left(\alpha^{L}, \beta^{L}, \mu_{1}\right)+(1-\lambda) j_{N-k}^{\mathrm{fr}, R^{L}}\left(\alpha^{L}, \beta^{L}, \mu_{2}\right)+\frac{k c}{N} \\
& j_{N}^{\mathrm{vr}}\left(\alpha^{L}, \beta^{L}, \lambda \mu_{1}+(1-\lambda) \mu_{2}\right) \\
& \quad \geq \lambda j_{N}^{\mathrm{vr}}\left(\alpha^{L}, \beta^{L}, \mu_{1}\right)+(1-\lambda) j_{N}^{\mathrm{vr}}\left(\alpha^{L}, \beta^{L}, \mu_{2}\right) \\
& j_{N}^{\mathrm{vr}}\left(\alpha^{L}, \beta^{L}, \lambda \mu_{1}+(1-\lambda) \mu_{2}\right) \\
& \quad \leq \lambda j_{N-k}^{\mathrm{vr}}\left(\alpha^{L}, \beta^{L}, \mu_{1}\right)+(1-\lambda) j_{N-k}^{\mathrm{vr}}\left(\alpha^{L}, \beta^{L}, \mu_{2}\right)+\frac{k c}{N}
\end{aligned}
$$

where $k=\left\lceil 1 / R_{1}\right\rceil$ and $c=\beta_{1}+\sum_{\ell=1}^{L} \alpha_{\ell}^{L} d^{*}$ are both independent of $N$. 
Proof: The proof given treats the fixed-rate- $R^{L}$ case. The proof for variable-rate codes follows the same argument. By (29) and the linearity of expectation,

$$
\begin{aligned}
& d_{\left(\alpha^{L}, \beta^{L}\right)}^{R^{L}}\left(\lambda \mu_{1}+(1-\lambda) \mu_{2}, \mathcal{C}^{L, N}\right) \\
& =\lambda E_{\mu_{1}} d_{\left(\alpha^{L}, \beta^{L}\right)}^{R^{L}}\left(X^{N}, \mathcal{C}^{L, N}\right) \\
& \quad+(1-\lambda) E_{\mu_{2}} d_{\left(\alpha^{L}, \beta^{L}\right)}^{R^{L}}\left(X^{N}, \mathcal{C}^{L, N}\right) \\
& \quad=\lambda d_{\left(\alpha^{L}, \beta^{L}\right)}^{R^{L}}\left(\mu_{1}, \mathcal{C}^{L, N}\right)+(1-\lambda) d_{\left(\alpha^{L}, \beta^{L}\right)}^{R^{L}}\left(\mu_{2}, \mathcal{C}^{L, N}\right)
\end{aligned}
$$

for any $\mathcal{C}^{L, N} \in \mathcal{K}^{\mathrm{fr}}(L, N)$. Hence, by Lemma 8 ,

$$
\begin{aligned}
j_{N}^{\mathrm{fr},}, R^{L} & \left(\alpha^{L}, \beta^{L}, \lambda \mu_{1}+(1-\lambda) \mu_{2}\right) \\
= & \inf _{\mathcal{C}^{L, N} \in \mathcal{K}^{\mathrm{fr}, R^{L}}(L, N)}\left[\lambda d_{\left(\alpha^{L}, \beta^{L}\right)}^{R^{L}}\left(\mu_{1}, \mathcal{C}^{L, N}\right)\right. \\
& \left.+(1-\lambda) d_{\left(\alpha^{L}, \beta^{L}\right)}^{R^{L}}\left(\mu_{2}, \mathcal{C}^{L, N}\right)\right] \\
& \geq \lambda j_{N}^{\mathrm{fr}, R^{L}}\left(\alpha^{L}, \beta^{L}, \mu_{1}\right)+(1-\lambda) j_{N}^{\mathrm{fr}, R^{L}}\left(\alpha^{L}, \beta^{L}, \mu_{2}\right)
\end{aligned}
$$

where $\mathcal{K}^{\mathrm{fr}, R^{L}}(L, N) \subseteq \mathcal{K}^{\mathrm{fr}}(L, N)$ again denotes the set of fixed-rate codes with rate vector less than $R^{L}$.

On the other hand, for $R_{1}>0, k=\left\lceil 1 / R_{1}\right\rceil$, and $\epsilon>0$, by Lemma 8 , there exist rate- $R^{L}$ codebooks

$$
\mathcal{C}^{L, N-k}(1), \mathcal{C}^{L, N-k}(2) \in \mathcal{K}^{\mathrm{fr}, R^{L}}(L, N-k)
$$

such that

$$
d_{\left(\alpha^{L}, \beta^{L}\right)}^{R^{L}}\left(\mu_{i}, \mathcal{C}^{L, N}(i)\right) \leq j_{N}^{\mathrm{fr}, R^{L}}\left(\alpha^{L}, \beta^{L}, \mu_{i}\right)+\epsilon, \quad i \in\{1,2\} .
$$

Notice that the rates of these two fixed-rate codes are both bounded by $R^{L}$ and that these rates are typically equal since more rate generally leads to lower distortion. Nonetheless, based on the definitions given here, the rates are not guaranteed to be identical. While identical rates are unnecessary for the variable-rate coding argument, they play a key role in the fixed-rate- $R^{L}$ coding argument. In this case, identical rates can be guaranteed by padding the binary descriptions of the lower rate code with 0's to achieve identical rates in the two fixed-rate codes. Notice that for fixed-rate- $R^{L}$ codes, $d_{\left(\alpha^{L}, \beta^{L}\right)}^{R^{L}}\left(\mu_{i}, \mathcal{C}^{L, N}(i)\right)$ is unchanged by this padding, and thus the new codebook is effectively identical to the old codebook for our purposes.

Let $\mathcal{C}^{L, N}$ be the codebook achieved by taking the union of the above two (identical rate) codebooks and padding each codeword with $k$ escape characters. Adding one to the description length of each codeword in $\mathcal{C}_{(1)}^{N}(1)$ and $\mathcal{C}_{(1)}^{N}(2)$ and leaving the description lengths of the other resolutions unchanged yields a code that satisfies the Kraft inequality. Then $\mathcal{C}^{L, N-k}(1), \mathcal{C}^{L, N-k}(2) \in \mathcal{K}^{\mathrm{fr}}(L, N-k)$ implies $\mathcal{C}^{L, N} \in$ $\mathcal{K}^{\text {fr }}(L, N)$. Finally, by the choice of $k, \mathcal{C}^{L, N}$ satisfies the $R^{L}$ rate constraint, giving $\mathcal{C}^{L, N} \in \mathcal{K}^{\mathrm{fr}, R^{L}}(L, N)$. Thus

$$
\begin{aligned}
& j_{N}^{\mathrm{fr}, R^{L}}\left(\alpha^{L}, \beta^{L}, \lambda \mu_{1}+(1-\lambda) \mu_{2}\right) \\
& \quad \leq d_{\left(\alpha^{L}, \beta^{L}\right)}^{R^{L}}\left(\lambda \mu_{1}+(1-\lambda) \mu_{2}, \mathcal{C}^{L, N}\right) \\
& \quad=\lambda d_{\left(\alpha^{L}, \beta^{L}\right)}^{R^{L}}\left(\mu_{1}, \mathcal{C}^{L, N}\right)+(1-\lambda) d_{\left(\alpha^{L}, \beta^{L}\right)}^{R^{L}}\left(\mu_{2}, \mathcal{C}^{L, N}\right)
\end{aligned}
$$

and

$$
\begin{aligned}
d_{\left(\alpha^{L}, \beta^{L}\right)}^{R^{L}}\left(\mu_{i}, \mathcal{C}^{L, N}\right) \leq & \frac{1}{N}\left(\alpha ^ { L } \left(\boldsymbol{D}\left(\mu_{i}, \mathcal{C}^{L, N-k}(i)\right)\right.\right. \\
& \left.\left.+k\left(d^{*}, \cdots, d^{*}\right)\right)^{t}+\beta^{L}\left(N R^{L}\right)^{t}\right)
\end{aligned}
$$

for $i \in\{1,2\}$ together imply

$$
\begin{aligned}
j_{N}^{\mathrm{fr},} & R^{L}\left(\alpha^{L}, \beta^{L}, \lambda \mu_{1}+(1-\lambda) \mu_{2}\right) \\
\leq & \lambda d_{\left(\alpha^{L}, \beta^{L}\right)}^{R^{L}}\left(\mu_{1}, \mathcal{C}^{L, N}(1)\right) \\
& \quad+(1-\lambda) d_{\left(\alpha^{L}, \beta^{L}\right)}\left(\mu_{2}, \mathcal{C}^{L, N}(2)\right)+\frac{k d^{*} \sum_{\ell=1}^{L} \alpha_{\ell}}{N} \\
& \leq \lambda j_{N-k}^{\mathrm{fr}, R^{L}}\left(\alpha^{L}, \beta^{L}, \mu_{1}\right)+(1-\lambda) j_{N-k}^{\mathrm{fr}, R^{L}}\left(\alpha^{L}, \beta^{L}, \mu_{2}\right)+\frac{k d^{*}}{N} .
\end{aligned}
$$

Notice that the above argument holds for $j^{\mathrm{fr}, R^{L}}(\cdot)$ and $j^{\mathrm{vr}}(\cdot)$ but fails for $j^{\text {fr }}(\cdot)$ since in this case codes $\mathcal{C}^{L, N-k}(1)$ and $\mathcal{C}^{L, N-k}(2)$ do not necessarily have the same rate vectors and thus the union code would not necessarily be a fixed-rate code. While the descriptions of the code with the smaller rate can be padded with 0 's to achieve identical description lengths in the two codes, such a padding increases $j^{\mathrm{fr}}\left(\alpha^{L}, \beta^{L}, \mu\right)$, and thus the argument cannot be carried forward.

Lemma 14 establishes the equivalence between the infimum and the limit of $N$ th-order weighted operational distortionrate functions. This result is achieved by proving that $N j_{N}^{\mathrm{fr}, R^{L}}\left(\alpha^{L}, \beta^{L}, \mu\right)$ and $N j_{N}^{\mathrm{vr}}\left(\alpha^{L}, \beta^{L}, \mu\right)$ are subadditive and then noting the equivalence of the limit and the infimum for normalized subadditive sequences.

Lemma 14: If $\mu$ is a stationary source, then

$$
\begin{aligned}
j^{\mathrm{fr}, R^{L}}\left(\alpha^{L}, \beta^{L}, \mu\right) & =\lim _{N \rightarrow \infty} j_{N}^{\mathrm{fr}, R^{L}}\left(\alpha^{L}, \beta^{L}, \mu\right) \\
j^{\mathrm{vr}}\left(\alpha^{L}, \beta^{L}, \mu\right) & =\lim _{N \rightarrow \infty} j_{N}^{\mathrm{vr}}\left(\alpha^{L}, \beta^{L}, \mu\right) .
\end{aligned}
$$

Proof: The proofs for the fixed-rate- $R^{L}$ and variablerate cases are almost identical. Again, only the details for the fixed-rate- $R^{L}$ case appear here. Fix $N, k \geq 1$, and $\epsilon>0$. By Lemma 8 there exist fixed-rate- $R^{L}$ quantizers $Q^{L, N} \in$ $\mathcal{Q}^{\mathrm{fr}, R^{L}}(L, N)$ and $Q^{L, k} \in \mathcal{Q}^{\mathrm{fr}, R^{L}}(L, k)$ with codebooks $\mathcal{C}^{L, N} \in \mathcal{K}^{\mathrm{fr}, R^{L}}(L, N)$ and $\mathcal{C}^{L, k} \in \mathcal{K}^{\mathrm{fr}, R^{L}}(L, k)$ such that

$$
d_{\left(\alpha^{L}, \beta^{L}\right)}^{R^{L}}\left(\mu, \mathcal{C}^{L, N}\right) \leq j_{N}^{\mathrm{fr}, R^{L}}\left(\alpha^{L}, \beta^{L}, \mu\right)+\epsilon
$$

and

$$
d_{\left(\alpha^{L}, \beta^{L}\right)}^{R^{L}}\left(\mu, \mathcal{C}^{L, k}\right) \leq j_{k}^{\mathrm{fr}, R^{L}}\left(\alpha^{L}, \beta^{L}, \mu\right)+\epsilon .
$$

Let $Q^{L, N+k}$ be the quantizer with codebook $\mathcal{C}^{L, N+k}$ such that quantizing $x^{N+k}=\left(x^{N}, x^{k}\right)$ with $Q^{L, N+k}$ is equivalent to quantizing $x^{N}$ and $x^{k}$ sequentially with quantizers $Q^{L, N}$ and $Q^{L, k}$. Then $Q^{L, N} \in \mathcal{Q}^{\mathrm{fr}, R^{L}}(L, N)$ and $Q^{L, k} \in \mathcal{Q}^{\mathrm{fr}, R^{L}}(L, k)$ imply $Q^{L, N+k} \in \mathcal{Q}^{\mathrm{fr}, R^{L}}(L, N+k)$. Thus

$$
\begin{aligned}
& (N+k) d_{\left(\alpha^{L}, \beta^{L}\right)}^{R^{L}}\left(\mathcal{C}^{L, N+k}, \mu\right) \\
& \quad=N d_{\left(\alpha^{L}, \beta^{L}\right)}^{R^{L}}\left(\mathcal{C}^{L, N}, \mu\right)+k d_{\left(\alpha^{L}, \beta^{L}\right)}^{R^{L}}\left(\mathcal{C}^{L, N}, \mu\right) \\
& \quad \leq N j_{N}^{\mathrm{fr}, R^{L}}\left(\alpha^{L}, \beta^{L}, \mu\right)+k j_{k}^{\mathrm{fr}, R^{L}}\left(\alpha^{L}, \beta^{L}, \mu\right)+(N+k) \epsilon
\end{aligned}
$$


for arbitrary $\epsilon$. Finally,

$$
j_{N+k}^{\mathrm{fr}, R^{L}}\left(\alpha^{L}, \beta^{L}, \mu\right) \leq d_{\left(\alpha^{L}, \beta^{L}\right)}^{R^{L}}\left(\mathcal{C}^{L, N+k}, \mu\right)
$$

implies

$$
\begin{aligned}
& (N+k) j_{N+k}^{\mathrm{fr}, R^{L}}\left(\alpha^{L}, \beta^{L}, \mu\right) \\
& \quad \leq N j_{N}^{\mathrm{fr}, R^{L}}\left(\alpha^{L}, \beta^{L}, \mu\right)+k j_{k}^{\mathrm{fr}, R^{L}}\left(\alpha^{L}, \beta^{L}, \mu\right) .
\end{aligned}
$$

That is, $\left\{N j_{N}^{\mathrm{fr}, R^{L}}\left(\alpha^{L}, \beta^{L}, \mu\right)\right\}$ is a subadditive sequence, which gives the desired result.

The above Lemmas combine to show that $j^{\mathrm{fr}, R^{L}}\left(\alpha^{L}, \beta^{L}, \mu\right)$ and $j^{\mathrm{vr}}\left(\alpha^{L}, \beta^{L}, \mu\right)$ are affine.

Lemma 15: The weighted operational distortion-rate functions are affine functions of $\mu \in \mathcal{P}_{s}$, the class of stationary sources.

Proof: Taking the limit in Lemma 13 and then applying the result from Lemma 14 gives the desired result.

The fact that $j^{\mathrm{fr}, R^{L}}\left(\alpha^{L}, \beta^{L}, \mu\right)$ and $j^{\mathrm{vr}}\left(\alpha^{L}, \beta^{L}, \mu\right)$ are upper semicontinuous remains to be shown. The following two lemmas address this goal.

Lemma 16 [21, Lemma 7.5.1]: Given a Polish measurable space $\left(A^{\infty}, \mathcal{B}^{\infty}\right)$ with countable generating field $\mathcal{G}=\left\{G_{i}\right.$ : $i=1,2, \ldots\}$, define a corresponding metric $d_{\mathcal{G}}$ on the space $\mathcal{P}\left(A^{\infty}, \mathcal{B}^{\infty}\right)$ of measures on $\left(A^{\infty}, \mathcal{B}^{\infty}\right)$ as

$$
d_{\mathcal{G}}\left(\mu_{1}, \mu_{2}\right)=\sum_{i=1}^{\infty} 2^{-i}\left|\mu_{1}\left(G_{i}\right)-\mu_{2}\left(G_{i}\right)\right|
$$

for any $\mu_{1}, \mu_{2} \in \mathcal{P}\left(A^{\infty}, \mathcal{B}^{\infty}\right)$. Let $f$ be a nonnegative continuous function. Then $d_{\mathcal{G}}\left(\mu_{n}, \mu\right) \rightarrow 0$ implies that

$$
\limsup _{n} E_{\mu_{\imath}} f \leq E_{\mu} f
$$

Lemma 17: If $\rho(x, y)$ is a nonnegative, continuous function of $x \in A$ for each $y \in \hat{A}$, then

$$
\begin{aligned}
d_{\left(\alpha^{L}, \beta^{L}\right)}^{R^{L}}\left(x^{N}, \mathcal{C}^{L, N}\right) & \\
& =(1 / N) \inf _{\boldsymbol{y}^{L} \in \mathcal{C}^{L, N}} \sum_{\ell=1}^{L}\left(\alpha_{\ell} \rho\left(x^{N}, \boldsymbol{y}_{\ell}\right)+\beta_{\ell} n R_{\ell}\right)
\end{aligned}
$$

is a nonnegative continuous function of $x^{N}$ for each $\mathcal{C}^{L, N} \in$ $\mathcal{K}^{\mathrm{fr}, R^{L}}(L, N)$ and, likewise,

$$
\begin{aligned}
d_{\left(\alpha^{L}, \beta^{L}\right)}^{R^{L}}\left(x^{N}, \mathcal{C}^{L, N}\right) & \\
& =(1 / N) \inf _{\boldsymbol{y}^{L} \in \mathcal{C}^{L, N}} \sum_{\ell=1}^{L}\left(\alpha_{\ell} \rho\left(x^{N}, \boldsymbol{y}_{\ell}\right)+\beta_{\ell}\left|\boldsymbol{y}_{\ell}\right|\right)
\end{aligned}
$$

is a nonnegative continuous function of $x^{N}$ for each $\mathcal{C}^{L, N} \in$ $\mathcal{K}^{\operatorname{vr}}(L, N)$.
Proof: This argument treats the variable-rate case. The parallel argument works for fixed-rate- $R^{L}$ codes. The goal is to show that, given any $\left\{\boldsymbol{x}_{n}\right\} \subseteq A^{N}$ such that $\boldsymbol{x}_{n} \rightarrow x^{N}$, the performance

$$
d_{\left(\alpha^{L}, \beta^{L}\right)}\left(\boldsymbol{x}_{n}, \mathcal{C}^{L, N}\right) \rightarrow d_{\left(\alpha^{L}, \beta^{L}\right)}\left(x^{N}, \mathcal{C}^{L, N}\right) .
$$

By Lemma 8 , the infimum defining $d_{\left(\alpha^{L}, \beta^{L}\right)}\left(x^{N}, \mathcal{C}^{L, N}\right)$ is achieved, and is actually a minimum, giving the desired result.

Proof of Theorems 5 and 7: It is sufficient to show that $j^{\mathrm{fr}, R^{L}}\left(\alpha^{L}, \beta^{L}, \mu\right)$ and $j^{\mathrm{vr}}\left(\alpha^{L}, \beta^{L}, \mu\right)$ satisfy the conditions of Lemma 12 .

1) Integrable. Since distortion vector $\left(d^{*}\right)^{L}$ is achievable at rate $\mathbf{0}$

$$
\begin{aligned}
j^{\mathrm{fr}, R^{L}}\left(\alpha^{L}, \beta^{L}, \mu_{x}\right) & \leq j_{N}^{\mathrm{fr}, R^{L}}\left(\alpha^{L}, \beta^{L}, \mu_{x}\right) \\
& \leq(1 / N) \sum_{\ell=1}^{L} \alpha_{\ell} E_{\mu_{x}} \rho\left(X^{N},\left(y_{1}^{*}\right)^{N}\right) \\
& \leq E_{\mu_{x}} \rho\left(X, y_{1}^{*}\right)
\end{aligned}
$$

for any $R^{L}$. Thus $j^{\mathrm{fr}, R^{L}}\left(\alpha^{L}, \beta^{L}, \mu_{x}\right)$ is integrable since $E_{\mu_{x}} \rho\left(X, y_{1}^{*}\right)$ is integrable. The same argument holds for $j^{\operatorname{vr}}\left(\alpha^{L}, \beta^{L}, \mu_{x}\right)$.

2) Affine. See Lemma 15.

3) Upper Semicontinuous. A Polish (by assumption) implies $A^{\infty}$ is Polish as well. Let $\mathcal{G}=\left\{G_{i}, i=1,2, \cdots\right\}$ be any countable generating field of $\mathcal{A}^{\infty}$, and for any $\mu_{1}, \mu_{2} \in \mathcal{P}\left(A^{\infty}, \mathcal{A}^{\infty}\right)$, the space of measures on $\left(A, \mathcal{A}^{\infty}\right)$, define the distance $d_{\mathcal{G}}\left(\mu_{1}, \mu_{2}\right)$ as

$$
d_{\mathcal{G}}\left(\mu_{1}, \mu_{2}\right)=\sum_{i=1}^{\infty} 2^{-i}\left|\mu_{1}\left(G_{i}\right)-\mu_{2}\left(G_{i}\right)\right|
$$

as in Lemma 16. Pick $N$ large enough so that

$$
j_{N}^{\mathrm{fr}, R^{L}}\left(\alpha^{L}, \beta^{L}, \mu\right) \leq j^{\mathrm{fr}, R^{L}}\left(\alpha^{L}, \beta^{L}, \mu\right)+\epsilon
$$

and choose $\mathcal{C}^{L, N} \in \mathcal{K}^{\mathrm{fr}, R^{L}}(L, N)$ such that

$$
d_{\left(\alpha^{L}, \beta^{L}\right)}^{R^{L}}\left(\mu, \mathcal{C}^{L, N}\right) \leq j_{N}^{\mathrm{fr}, R^{L}}\left(\alpha^{L}, \beta^{L}, \mu\right)+\epsilon .
$$

Then given some $\left\{\mu_{n}, n=1,2, \cdots\right\}$ such that $d_{\mathcal{G}}\left(\mu_{n}, \mu\right) \rightarrow 0$ as $n \rightarrow \infty$

$$
\begin{aligned}
\limsup _{n} f^{\mathrm{fr}, R^{L}}\left(\alpha^{L}, \beta^{L}, \mu_{n}\right) & \leq \limsup _{n} j_{N}^{\mathrm{fr}, R^{L}}\left(\alpha^{L}, \beta^{L}, \mu_{n}\right) \\
& \leq \underset{n}{\limsup } d_{\left(\alpha^{L}, \beta^{L}\right)}^{R^{L}}\left(\mathcal{C}^{L, N}, \mu_{n}\right) \\
& \leq E_{\mu^{N}} d_{\left(\alpha^{L}, \beta^{L}\right)}^{R^{L}}\left(X^{N}, \mathcal{C}^{L, N}\right)
\end{aligned}
$$

by Lemmas 16 and 17

$$
\begin{aligned}
& \leq j_{N}^{\mathrm{fr}, R^{L}}\left(\alpha^{L}, \beta^{L}, \mu\right)+\epsilon \\
& \leq j^{\mathrm{fr}, R^{L}}\left(\alpha^{L}, \beta^{L}, \mu\right)+2 \epsilon
\end{aligned}
$$

for arbitrary $\epsilon$. Thus $j^{\mathrm{fr}, R^{L}}\left(\alpha^{L}, \beta^{L}, \mu\right)$ is upper semicontinuous for fixed $\alpha^{L}, \beta^{L} \geq \mathbf{0}$. The same argument holds for $j^{\mathrm{vr}}\left(\alpha^{L}, \beta^{L}, \mu\right)$.

Next consider the ergodic decomposition of $J\left(\alpha^{L}, \beta^{L}, \mu\right)$. 
Proof of Theorem 8: By the Converse given in Lemma $7, j^{\operatorname{vr}}\left(\alpha^{L}, \beta^{L}, \mu\right) \geq J\left(\alpha^{L}, \beta^{L}, \mu\right)$. Thus by Theorems 7 and 3

$$
\begin{aligned}
J\left(\alpha^{L}, \beta^{L}, \mu\right) & \leq \int j^{\mathrm{vr}}\left(\alpha^{L}, \beta^{L}, \mu_{x}\right) d \mu(x) \\
& =\int J\left(\alpha^{L}, \beta^{L}, \mu_{x}\right) d \mu(x)
\end{aligned}
$$

since $\mu_{x}$ is ergodic for each $x \in A^{\infty}$. On the other hand, for any $N \geq 1$, by the concavity in $\mu_{x}$ of $I_{\mu_{x} q}\left(X^{N} ; \boldsymbol{Y}_{\ell} \mid \boldsymbol{Y}^{\ell-1}\right)$ [25]

$$
\begin{aligned}
& \int J_{N}\left(\alpha^{L}, \beta^{L}, \mu_{x}\right) d \mu(x) \\
& =\int\left[\operatorname { i n f } _ { q } \frac { 1 } { N } \sum _ { \ell = 1 } ^ { L } \left(\alpha_{\ell} E_{\mu_{x}^{N} q^{N}} \rho\left(X^{N}, \boldsymbol{Y}_{\ell}\right)\right.\right. \\
& \left.\left.+\beta_{\ell} I_{\mu_{x}^{N} q^{N}}\left(X^{N} ; \boldsymbol{Y}_{\ell} \mid \boldsymbol{Y}^{\ell-1}\right)\right)\right] d \mu(x) \\
& \leq \inf _{q} \int\left[\frac { 1 } { N } \sum _ { \ell = 1 } ^ { L } \left(\alpha_{\ell} E_{\mu_{x}^{N} q^{N} \rho\left(X^{N}, \boldsymbol{Y}_{\ell}\right)}\right.\right. \\
& \left.\left.\quad+\beta_{\ell} I_{\mu_{x}^{N} q^{N}}\left(X^{N} ; \boldsymbol{Y}_{\ell} \mid \boldsymbol{Y}^{\ell-1}\right)\right)\right] d \mu(x) \\
& \leq \inf _{q} \frac{1}{N} \sum_{\ell=1}^{L}\left(\alpha_{\ell} E_{\mu^{N} q^{N} \rho\left(X^{N}, \boldsymbol{Y}_{\ell}\right)}\right. \\
& \left.+\beta_{\ell} I_{\mu^{N} q^{N}}\left(X^{N} ; \boldsymbol{Y}_{\ell} \mid \boldsymbol{Y}^{\ell-1}\right)\right) \\
& =J_{N}\left(\alpha^{L}, \beta^{L}, \mu\right),
\end{aligned}
$$

and thus

$$
\inf _{N} \int J_{N}\left(\alpha^{L}, \beta^{L}, \mu_{x}\right) d \mu(x) \leq J\left(\alpha^{L}, \beta^{L}, \mu\right) .
$$

Finally, Fatou's Lemma gives us

$$
\begin{aligned}
\int J\left(\alpha^{L}, \beta^{L}, \mu_{x}\right) d \mu(x) & =\int \inf _{N} J_{N}\left(\alpha^{L}, \beta^{L}, \mu_{x}\right) d \mu(x) \\
& \leq \inf _{N} \int J_{N}\left(\alpha^{L}, \beta^{L}, \mu_{x}\right) d \mu(x) \\
& \leq J\left(\alpha^{L}, \beta^{L}, \mu\right)
\end{aligned}
$$

giving the desired result.

\section{APPENDIX}

Proof of Lemma 1: The proof given treats $\mathcal{R}^{\mathrm{fr}, L}(\mu)$. The proof of the corresponding results for $\mathcal{D}^{\mathrm{fr}, L}\left(R^{L}, \mu\right)$ and $\mathcal{R}^{\mathrm{vr}, L}(\mu)$ are similar. Notice that for any $\left\{S_{N}\right\}$, $x \in \limsup _{N} S_{N}$ implies that for any $M \geq 1$ there exists $N \geq M$ such that $x \in S_{N}$. Thus $x \in \limsup _{N} S_{N}$ implies $x \in S_{N}$ infinitely often. Similarly, $x \in \liminf _{N} S_{N}$ implies that there exists $M \geq 1$ such that $x \in S_{N}$ for all $N \geq M$. Thus $x \in \liminf _{N} S_{N}$ implies that $x \in S_{N}$ eventually. Clearly, $x \in S_{N}$ eventually implies $x \in S_{N}$ infinitely often. Thus liminf ${ }_{N} S_{N} \subseteq \limsup { }_{N} S_{N}$ for any $\left\{S_{N}\right\}$. Further, if it can be shown that for any $x$ such that $x \in S_{N}$ for some $N \geq 1$, $x \in S_{N}$ eventually, then $\limsup _{N} S_{N} \subseteq \liminf _{N} S_{N}$, and $\lim _{N} S_{N}=\limsup S_{N} S_{N}=\liminf _{N} S_{N}$.
Given the above discussion, it is sufficient to show that for any $\left(r^{L}, d^{L}\right) \in \mathcal{R}_{N_{1}}^{\mathrm{fr}, L}(\mu)$ for some $N_{1} \geq 1$, there exists $M \geq 1$ such that $\left(r^{L}, d^{L}\right) \in \mathcal{R}_{N}^{\mathrm{fr}, L}(\mu)$ for all $N \geq M$. From the definition of the achievable distortion-rate region, $\left(r^{L}, d^{L}\right) \in \mathcal{R}_{N_{1}}^{\mathrm{fr}, L}(\mu)$ implies that for any $\epsilon>0$, there exists $Q^{L, N_{1}} \in \mathcal{Q}^{\mathrm{fr}}\left(L, N_{1}\right)$ such that

$$
\left(1 / N_{1}\right)\left(\boldsymbol{R}\left(\mu, Q^{L, N_{1}}\right), \boldsymbol{D}\left(\mu, Q^{L, N_{1}}\right)\right) \leq\left(r^{L}, d^{L}\right)+(\epsilon, \cdots, \epsilon) .
$$

Let $\left\{\mathcal{C}_{(1)}^{N_{1}}, \cdots, \mathcal{C}_{(L)}^{N_{1}}\right\}$ be the $L, N_{1}$-dimensional codebooks associated with $Q^{L, N_{1}}$. Then for any $N>N_{1}$, construct a collection of $N$-dimensional codebooks $\left\{\mathcal{C}_{(1)}^{N}, \cdots, \mathcal{C}_{(L)}^{N}\right\}$ with codewords created by concatenating some collection of $N_{1}$ dimensional codewords from the $L$ codebooks of $Q^{L, N_{1}}$ and then padding with the reference letter $y_{1}^{*}$ to reach dimension $N$. In particular, let $\left(k_{1}, k_{2}\right)$ be the unique pair of integers such that $k_{1} \geq 0,0 \leq k_{2}<N_{1}$, and $N=k_{1} N_{1}+k_{2}$. The first codebook is defined as

$$
\begin{gathered}
\mathcal{C}_{(1)}^{N}=\left\{\left(\left(\boldsymbol{y}_{1} \boldsymbol{y}_{2} \cdots \boldsymbol{y}_{k_{1}}\left(y_{1}^{*}\right)^{k_{2}}\right), \sum_{i=1}^{k_{1}}\left|\boldsymbol{y}_{i}\right|\right):\right. \\
\left.\boldsymbol{y}_{i} \in \mathcal{C}_{(1)}^{N_{1}} \forall 1 \leq i \leq k_{1}\right\} .
\end{gathered}
$$

Thus each codeword in $\mathcal{C}_{(1)}^{N}$ is made by concatenating $k_{1}$ codewords from $\mathcal{C}_{(1)}^{N_{1}}$ followed by $k_{2}$ copies of the reference letter $y_{1}^{*}$. For each successive $\ell \in\{2, \cdots, L\}$

$$
\mathcal{C}_{(\ell)}^{N}=\bigcup_{z^{N} \in \mathcal{C}_{(\ell-1)}^{N}} \mathcal{C}_{(\ell)}^{N}\left(z^{N}\right)
$$

where for any $z^{N} \in \mathcal{C}_{(\ell-1)}^{N}$

$$
\begin{aligned}
\mathcal{C}_{(\ell)}^{N}\left(z^{N}\right)= & \left\{\left(\left(\boldsymbol{y}_{1} \boldsymbol{y}_{2} \cdots \boldsymbol{y}_{k_{1}}\left(y_{1}^{*}\right)^{k_{2}}\right), \sum_{i=1}^{k_{1}}\left|\boldsymbol{y}_{i}\right|\right):\right. \\
& \left.\boldsymbol{y}_{i} \in \mathcal{C}_{(\ell)}^{N_{1}}\left(z_{(i-1) N_{1}+1}^{i N_{1}}\right) \forall 1 \leq i \leq k_{1}\right\} .
\end{aligned}
$$

Finally, for any $x^{N} \in A^{N}$, define $Q^{L, N}$ such that $Q^{L, N}\left(x^{N}\right)=y^{N}$ implies

$$
y_{(i-1) N_{1}+1}^{i N}=Q^{L, N_{1}}\left(x_{(i-1) N_{1}+1}^{i N_{1}}\right)
$$

for all $1 \leq i \leq k_{1}$ and $y_{i}=y_{1}^{*}$ for all $i>k_{1} N_{1}$. The resulting code is called a concatenation code.

If $Q^{L, N}$ is a concatenation code built from $k_{1}$ copies of $Q^{L, N_{1}}$, encoding $x^{N}$ with $Q^{L, N}$ is equivalent to independently encoding the first $k_{1} N_{1}$-dimensional subvectors of $x^{N}$ with $Q^{L, N_{1}}$ and then coding all $k_{2}$ remaining symbols to $y_{1}^{*}$. Thus if $Q^{L, N_{1}}$ is a uniquely decodable, fixed-rate code of dimension $N_{1}, Q^{L, N}$ is a uniquely decodable, fixed-rate code of dimension $N$, and thus $Q^{L, N} \in \mathcal{Q}^{\mathrm{fr}}(L, N)$. For any stationary $\mu$

$$
\frac{1}{N} \boldsymbol{R}\left(\mu, Q^{L, N}\right)=\frac{k_{1}}{N} \boldsymbol{R}\left(\mu, Q^{L, N_{1}}\right) \leq r^{L}+(\epsilon, \cdots, \epsilon)
$$



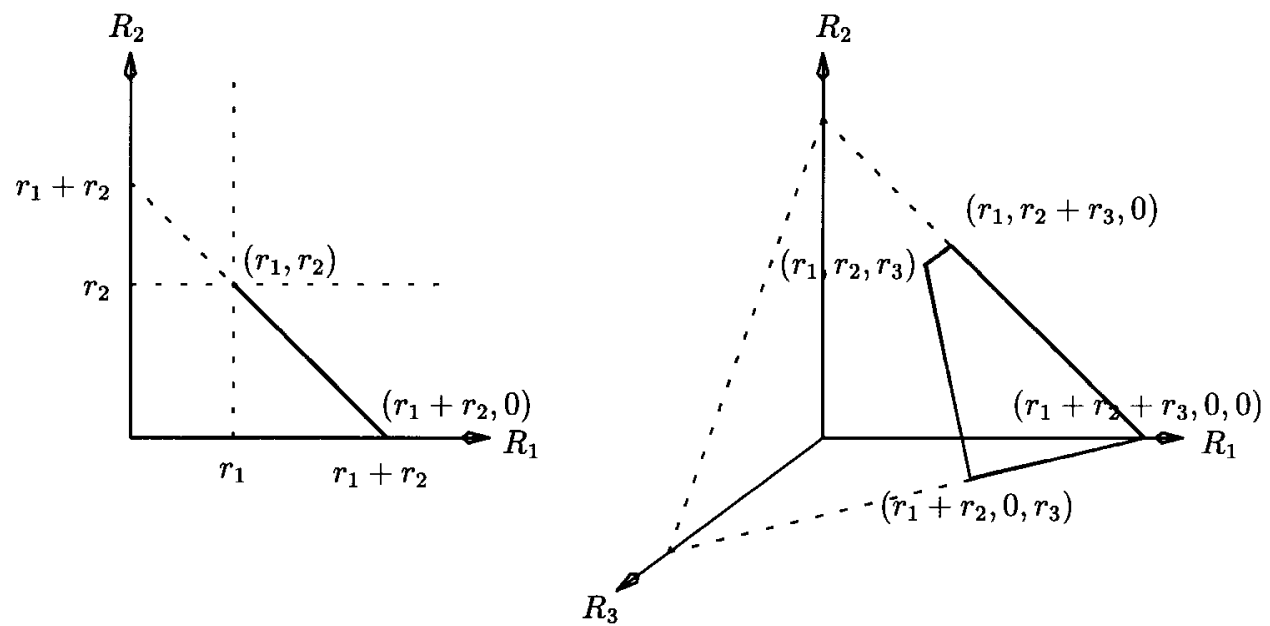

Fig. 6. Extrema defining the region of rates described by Lemma 3 for $L=2$ (left) and $L=3$ (right).

and

$$
\begin{aligned}
\frac{1}{N} \boldsymbol{D}\left(\mu, Q^{L, N}\right) & =\frac{1}{N}\left(k_{1} \boldsymbol{D}\left(\mu, Q^{L, N_{1}}\right)+k_{2}\left(d^{*}, \cdots, d^{*}\right)\right) \\
& \leq d^{L}+(2 \epsilon, \cdots, 2 \epsilon)
\end{aligned}
$$

for $N$ large enough since $k_{2}$ is bounded and the escape distortion $d^{*}<\infty$. Finally, $\epsilon$ arbitrary gives us the desired result.

Proof of Lemma 2: Again, focus on $\mathcal{R}^{\mathrm{fr}, L}(L, \mu)$. The proofs for $\mathcal{D}^{\mathrm{fr}, L}\left(R^{L}, \mu\right)$ and $\mathcal{R}^{\mathrm{vr}, L}(\mu)$ are almost identical. For any

$$
\left(r_{(1)}^{L}, d_{(1)}^{L}\right),\left(r_{(2)}^{L}, d_{(2)}^{L}\right) \in \mathcal{R}^{\mathrm{fr}, L}(\mu)
$$

and any $\lambda \in[0,1]$, let

$$
\left(r^{L}, d^{L}\right)=\lambda\left(r_{(1)}^{L}, d_{(1)}^{L}\right)+(1-\lambda)\left(r_{(2)}^{L}, d_{(2)}^{L}\right) .
$$

The question is whether or not $\left(r^{L}, d^{L}\right)$ falls within $\mathcal{R}^{\mathrm{fr}, L}(\mu)$. Combining the definitions of $\mathcal{R}^{\mathrm{fr}, L}(\mu)$ and $\mathcal{R}_{N}^{\mathrm{fr}, L}(\mu)$ with Lemma 1

$$
\left(r_{(1)}^{L}, d_{(1)}^{L}\right),\left(r_{(2)}^{L}, d_{(2)}^{L}\right) \in \mathcal{R}^{\mathrm{fr}, L}(\mu)
$$

implies that for any $\epsilon>0$ there exists an integer $M$ such that for any $N \geq M, N_{1}=\lfloor\lambda N\rfloor$, and $N_{2}=N-N_{1}$ there exist quantizers $\bar{Q}^{L, N_{1}} \in \mathcal{Q}^{\mathrm{fr}}\left(L, N_{1}\right)$ and $Q^{L, N_{2}} \in \mathcal{Q}^{\mathrm{fr}}\left(L, N_{2}\right)$ such that

$$
\begin{aligned}
& \frac{1}{N_{1}}\left(\boldsymbol{R}\left(Q^{L, N_{1}}, \mu\right), \boldsymbol{D}\left(Q^{L, N_{1}}, \mu\right)\right) \leq\left(r_{(1)}^{L}, d_{(1)}^{L}\right)+(\epsilon, \cdots, \epsilon) \\
& \frac{1}{N_{2}}\left(\boldsymbol{R}\left(Q^{L, N_{2}}, \mu\right), \boldsymbol{D}\left(Q^{L, N_{2}}, \mu\right)\right) \leq\left(r_{(2)}^{L}, d_{(2)}^{L}\right)+(\epsilon, \cdots, \epsilon) .
\end{aligned}
$$

(For any $r \in \mathbb{R},\lfloor r\rfloor$ is the largest integer not exceeding $r$.)

Then for any $N \geq M$, design an $N$-dimensional quantizer by concatenating codewords from $Q^{L, N_{1}}$ and $Q^{L, N_{2}}$. The argument parallels the argument of Lemma 1. In this case, coding any $x^{N}$ with the resulting $N$-dimensional codebook is equivalent to coding the first $N_{1}$ symbols of $x^{N}$ with $Q^{L, N_{1}}$ and coding the last $N_{2}$ symbols of $x^{N}$ with $Q^{L, N_{2}}$. To construct $Q^{L, N}$, first construct its $L$ codebooks, here denoted $\left\{\mathcal{C}_{(1)}^{N}, \cdots, \mathcal{C}_{(L)}^{N}\right\}$. Let $\left\{\mathcal{C}_{(1)}^{N_{1}}, \cdots, \mathcal{C}_{(L)}^{N_{1}}\right\}$ and $\left\{\mathcal{C}_{(1)}^{N_{2}}, \cdots, \mathcal{C}_{(L)}^{N_{2}}\right\}$ be the codebooks associated with $Q^{L, N_{1}}$ and $Q^{L, N_{2}}$ respectively. Then let

$$
\mathcal{C}_{(1)}^{N}=\left\{\left(\left(\boldsymbol{y}_{1} \boldsymbol{y}_{2}\right),\left|\boldsymbol{y}_{1}\right|+\left|\boldsymbol{y}_{2}\right|\right): \boldsymbol{y}_{1} \in \mathcal{C}_{(1)}^{N_{1}}, \boldsymbol{y}_{2} \in \mathcal{C}_{(1)}^{N_{2}}\right\} .
$$

For each successive $\ell \in\{2, \cdots, L\}$, let

$$
\mathcal{C}_{(\ell)}^{N}=\bigcup_{z^{N} \in \mathcal{C}_{(\ell-1)}^{N}} \mathcal{C}_{(\ell)}^{N}\left(z^{N}\right)
$$

where for any $z^{N} \in \mathcal{C}_{(\ell-1)}^{N}$

$$
\begin{aligned}
\mathcal{C}_{(\ell)}^{N}\left(z^{N}\right)=\{ & \left\{\left(\boldsymbol{y}_{1} \boldsymbol{y}_{2}\right),\left|\boldsymbol{y}_{1}\right|+\left|\boldsymbol{y}_{2}\right|\right): \boldsymbol{y}_{1} \in \mathcal{C}_{(\ell)}^{N_{1}}\left(z_{1}^{N_{1}}\right), \\
& \left.\boldsymbol{y}_{2} \in \mathcal{C}_{(\ell)}^{N_{2}}\left(z_{N_{1}+1}^{N}\right)\right\} .
\end{aligned}
$$

Finally, for any $x^{N} \in A^{N}$, define $Q^{L, N}$ such that $Q^{L, N}\left(x^{N}\right)=y^{N}$ implies

$$
y_{1}^{N_{1}}=Q^{L, N_{1}}\left(x_{1}^{N_{1}}\right)
$$

and

$$
y_{N_{1}+1}^{N}=Q^{L, N_{2}}\left(x_{N_{1}+1}^{N}\right) .
$$

The resulting fixed-rate, uniquely decodable code $Q^{L, N} \in$ $\mathcal{Q}^{\mathrm{fr}}(L, N)$ is another example of a concatenation code.

For any stationary $\mu$

$$
\begin{aligned}
\frac{1}{N} R\left(\mu, Q^{L, N}\right) & =\frac{1}{N}\left(\boldsymbol{R}\left(\mu, Q^{L, N_{1}}\right)+\boldsymbol{R}\left(\mu, Q^{L, N_{2}}\right)\right) \\
& \leq r^{L}+\frac{1}{N} r_{(2)}^{L}+(\epsilon, \cdots, \epsilon)
\end{aligned}
$$

and

$$
\begin{aligned}
\frac{1}{N} D\left(\mu, Q^{L, N}\right) & =\frac{1}{N}\left(\boldsymbol{D}\left(\mu, Q^{L, N_{1}}\right)+\boldsymbol{D}\left(\mu, Q^{L, N_{2}}\right)\right) \\
& \leq d^{L}+\frac{1}{N} d_{(2)}^{L}+(\epsilon, \cdots, \epsilon) .
\end{aligned}
$$

Thus there exists a sequence of codes $\left\{Q^{L, N} \in \mathcal{Q}^{\mathrm{fr}}(L, N)\right\}$ such that

$$
\frac{1}{N}\left(\boldsymbol{R}\left(\mu, Q^{L, N}\right), \boldsymbol{D}\left(\mu, Q^{L, N}\right)\right) \leq\left(r^{L}, d^{L}\right)+2(\epsilon, \cdots, \epsilon)
$$

for $N$ large enough and arbitrary $\epsilon$, giving the desired result. 
Proof of Lemma 3: Again, the proof given treats the fixed-rate case. The variable-rate case is similarly achieved. By definition of $\mathcal{R}^{\mathrm{fr}, L}(\mu)$, it is sufficient to show that $\left(r^{L}, d^{L}\right) \in$ $\mathcal{R}^{\mathrm{fr}, L}(\mu), \sum_{i=1}^{L} R_{i}=\sum_{i=1}^{L} r_{i}$, and $\sum_{i=1}^{\ell} R_{i} \geq \sum_{i=1}^{\ell} r_{i}$ for all $1 \leq \ell<L$ imply $\left(R^{L}, d^{L}\right) \in \mathcal{R}^{\mathrm{fr}, L}(\mu)$. The above constraints describe a convex subset of an $L$-dimensional hyperplane with $2^{L-1}$ extreme points, as shown in Fig. 6 for $L=2$ and $L=3$. Thus by convexity of $\mathcal{R}^{\mathrm{fr}, L}(\mu)$, proving the achievability of the extrema is sufficient for proving the achievability of every point in the region described by these constraints. For any $k \in\left\{0, \cdots, 2^{L-1}-1\right\}$, let $b_{k, i}$ be the $i$ th most significant digit in the natural $L$-bit binary expansion of $k$. (Thus $b_{k, 1}=0$ for all $k \in\left\{0, \cdots, 2^{L-1}-1\right\}$.) For each $k \in\left\{0, \cdots, 2^{L-1}-1\right\}$ and each $i \in\{1, \cdots, L\}$, let

$$
R_{k, i}=\left(1-b_{k, i}\right) r_{i}+\sum_{j=i+1}^{L} r_{j}-\sum_{j=i+1}^{L} R_{k, j}
$$

and set $\boldsymbol{R}_{k}=\left(R_{k, 1}, \cdots, R_{k, L}\right)$. Then $\left\{\boldsymbol{R}_{0}, \cdots, \boldsymbol{R}_{2^{L-1}-1}\right\} \subseteq$ $\mathbb{R}^{L}$ describes the above mentioned extrema. Achievability of these extrema is clear by inspection, since each rate vector can be achieved by a sequence of steps whereby a set of consecutive resolutions is concatenated into a single resolution in the position of the earliest of the resolutions in that collection. For example, if $\left(\left(r_{1}, r_{2}\right),\left(d_{1}, d_{2}\right)\right) \in \mathcal{R}^{\mathrm{fr}, L}(\mu)$, then $\left(\left(r_{1}+r_{2}, 0\right),\left(d_{1}, d_{2}\right)\right) \in \mathcal{R}^{\mathrm{fr}, L}(\mu)$, since concatenating the two resolutions of any code achieving the first constraint (to arbitrary accuracy) into a single first-resolution code and sending 0 bits in the second resolution gives a legitimate code achieving performance no worse than $\left(\left(r_{1}+r_{2}, 0\right),\left(d_{1}, d_{2}\right)\right)$ since both the first-resolution decoder and the second-resolution decoder of the new code may be designed to give reproductions identical to those of the code achieving $\left(\left(r_{1}, r_{2}\right),\left(d_{1}, d_{2}\right)\right)$. (In this case, the first-resolution decoder ignores all but the first $r_{1}$ bits per symbol while the second-resolution decoder decodes the entire rate $r_{1}+r_{2}$ description.)

\section{REFERENCES}

[1] E. A. Riskin and R. M. Gray, "A greedy tree growing algorithm for the design of variable rate vector quantizers," IEEE Trans. Signal Processing, vol. 39, pp. 2500-2507, Nov. 1991

[2] W. B. Pennebaker and J. L. Mitchell, JPEG Still Image Compression Standard. New York: Van Nostrand Reinhold, 1993.

[3] J. M. Shapiro, "Embedded image coding using zerotrees of wavelet coefficients," IEEE Trans. Signal Processing, vol. 41, pp. 3445-3462, Dec. 1993.
[4] A. Said and W. A. Pearlman, "A new, fast, and efficient image codec based on set partitioning in hierarchical trees," IEEE Trans. Circuits Syst. Video Technol., vol. 6, pp. 243-250, June 1996.

[5] T. Berger, Rate Distortion Theory: A Mathematical Basis for Data Compression. Englewood Cliffs, NJ: Prentice-Hall, 1971.

[6] M. Effros, P. A. Chou, and R. M. Gray, "Variable-rate source coding theorems pgfor stationary nonergodic sources," IEEE Trans. Inform. Theory, vol. 40, pp. 1920-1925, Nov. 1994.

[7] R. M. Gray and A. D. Wyner, "Source coding for a simple network," Bell Syst. Tech. J., vol. 53, no. 9, pp. 1681-1721, Nov. 1974.

[8] V. Koshelev, "Hierarchical coding of discrete sources," Probl. Pered. Inform., vol. 16, no. 3, pp. 31-49, 1980.

[9] , "An evaluation of the average distortion for discrete schemes of sequential approximation," Probl. Pered. Inform., vol. 17, no. 3, pp. 20-33, 1981

[10] R. M. Gray, "A new class of lower bounds to information rates via conditional rate-distortion functions," IEEE Trans. Inform. Theory, vol. IT-19, pp. 480-489, July 1973.

[11] A. El Gamal and T. M. Cover, "Achievable rates for multiple descriptions," IEEE Trans. Inform. Theory, vol. IT-28, pp. 851-857, Nov. 1982.

[12] R. Ahlswede, "The rate-distortion region for multiple descriptions without excess rate," IEEE Trans. Inform. Theory, vol. IT-31, pp. 721-726, Nov. 1985.

[13] W. H. R. Equitz, "Successive refinement of information," Ph.D. dissertation, Stanford Univ., Stanford, CA, 1989.

[14] W. H. R. Equitz and T. M. Cover, "Successive refinement of information," IEEE Trans. Inform. Theory, vol. 37, pp. 269-275, Mar. 1991.

[15] J. Chow and T. Berger, "Failure of succesive refinement for symmetrical Gaussian mixtures," IEEE Trans. Inform. Theory, vol. 43, pp. 350-352, Jan. 1997.

[16] B. Rimoldi, "Successive refinement of information: Characterization of achievable rates," IEEE Trans. Inform. Theory, vol. 40, pp. 253-259, Jan. 1994.

[17] P. A. Chou, T. Lookabaugh, and R. M. Gray, "Entropy-constrained vector quantization," IEEE Trans. Acoust., Speech Signal Processing, vol. 37, pp. 31-42, Jan. 1989.

[18] M. Effros, "Practical multiresolution source coding: TSVQ revisited," in Proc. IEEE Data Compression Conf. (Snowbird, UT, Mar. 1998), pp. $53-62$.

[19] M. Effros and D. Dugatkin, "Multi-resolution vector quantization," 1999, in preparation.

[20] D. G. Luenberger, Optimization by Vector Space Methods. New York: Wiley, 1969.

[21] R. M. Gray, Probability, Random Processes, and Ergodic Properties. New York: Springer-Verlag, 1988.

[22] R. M. Gray and L. D. Davisson, "Source coding theorems without the ergodic assumption," IEEE Trans. Inform. Theory, vol. IT-20, pp. 502-526, July 1974

[23] P. C. Shields, D. L. Neuhoff, L. D. Davisson, and F. Ledrappier, "The distortion-rate function for nonergodic sources," Ann. Probab., vol. 6, no. 1, pp. 138-143, 1978.

[24] A. M. Gerrish, "Estimation of information rates," Ph.D. dissertation, Yale Univ., New Haven, CT, 1963.

[25] T. M. Cover and J. A. Thomas, Elements of Information Theory. New York: Wiley, 1991.

[26] R. M. Gray, Entropy and Information Theory. New York: SpringerVerlag, 1990 